\title{
Roadmap on optofluidics
}

Paolo Minzioni ${ }^{1 *}$, Roberto Osellame ${ }^{2}$, Cinzia Sada $^{3}$, S. Zhao ${ }^{4}$, F. G. Omenetto ${ }^{4}$, Kristinn B. Gylfason ${ }^{5}$, Tommy Haraldsson ${ }^{5}$, Yibo Zhang ${ }^{6}$, Aydogan Ozcan ${ }^{6,7}$, Adam Wax ${ }^{8}$, Frieder Mugele ${ }^{9}$, Holger Schmidt ${ }^{10}$, Genni Testa $^{11}$, Romeo Bernini ${ }^{11}$, Jochen Guck ${ }^{12}$, Carlo Liberale ${ }^{13}$, Kirstine Berg-Sørensen ${ }^{14}$, Jian Chen ${ }^{15}$, Markus Pollnau ${ }^{16}$, Sha Xiong ${ }^{17}$, Ai-Qun Liu ${ }^{17}$, Chia-Chann Shiue ${ }^{18}$, Shih-Kang Fan ${ }^{18}$, David Erickson ${ }^{19}$ and David Sinton ${ }^{20}$

\section{Affiliations}

${ }^{1}$ Department of Electrical, Computer and Biomedical Engineering, University of Pavia, Via Ferrata 5A, 27100 Pavia, Italy

${ }^{2}$ Istituto di Fotonica e Nanotecnologie - CNR, P.za Leonardo da Vinci 32, I-20133 Milano, Italy

${ }^{3}$ Dipartimento di Fisica e Astronomia G. Galilei, Università di Padova, Via Marzolo 8, 35131, Padova, Italy

${ }^{4}$ Department of Biomedical Engineering, Tufts University, Medford, Massachusetts 02155, USA

${ }^{5}$ Micro and Nanosystems, KTH Royal Institute of Technology, Osquldas väg 10, SE-100 44 Stockholm, Sweden

${ }^{6}$ Electrical Engineering Department, Bioengineering Department, and California NanoSystems Institute (CNSI), University of California, Los Angeles, California 90095, USA

${ }^{7}$ Department of Surgery, David Geffen School of Medicine, University of California, Los Angeles, California 90095, USA

${ }^{8}$ Department of Biomedical Engineering, Duke University, Durham, NC 27708, USA

${ }^{9}$ Physics of Complex Fluids Group, MESA+ Institute, University of Twente, Enschede, The Netherlands

${ }^{10}$ School of Engineering, University of California Santa Cruz, 1156 High Street, Santa Cruz, California 95064, USA

${ }^{11}$ Istituto per il Rilevamento Elettromagnetico de ll’Ambiente, National Research Council, Via Diocleziano 328, 81031 Naples, Italy

${ }^{12}$ Biotechnology Center, Technische Universität Dresden, Am Tatzberg 47-49, 01307 Dresden, Germany

${ }^{13}$ Biological and Environmental Science and Engineering Division, King Abdullah University of Science and Technology (KAUST), Thuwal 23955-6900, Saudi Arabia

${ }^{14}$ Department of Physics, Technical University of Denmark, DK-2800 Kongens Lyngby, Denmark

${ }^{15}$ State Key Laboratory of Transducer Technology, Institute of Electronics, Chinese Academy of Sciences, Beijing 100190, China

${ }^{16}$ Department of Materials and Nano Physics, School of Information and Communication Technology, KTH-Royal Institute of Technology, Electrum 229, Isafjordsgatan 22-24, 16440 Kista, Sweden

${ }^{17}$ School of Electrical and Electronic Engineering, Nanyang Technological University, 50 Nanyang Avenue, Singapore 639798

${ }^{18}$ Department of Mechanical Engineering, National Taiwan University, Taipei 10617, Taiwan 
${ }^{19}$ Sibley School of Mechanical and Aerospace Engineering, Cornell Unversity, Ithaca, New York 14853, USA

${ }^{20}$ Department of Mechanical and Industrial Engineering, University of Toronto, 5 King’s College Road, Toronto, Ontario M5S 3G8, Canada

*Guest editor of the roadmap

Email: paolo.minzioni@unipv.it

\begin{abstract}
Optofluidics, nominally the research area where optics and fluidics merge, is a relatively new research field and it is only in the last decade that there has been a large increase in the number of optofluidics applications as well as in the number of research groups devoted to the topic. Nowadays optofluidics applications include, without being limited to, lab-on-chip devices, fluid-based and controlled lenses, optical sensors for fluids and for suspended particles, biosensors, imaging tools, etc. The long list of potential optofluidics applications, which have been recently demonstrated, suggests that optofluidic technologies will become more and more common in everyday life in the future, causing a significant impact on many aspects of our society.

A characteristic of this research field, deriving from both its inter-disciplinary origin and applications, is that in order to develop suitable solutions it is often required to combine a deep knowledge in different fields, ranging from materials science to photonics, from microfluidics to molecular biology and biophysics. As a direct consequence, also being able to understand the long-term evolution of optofluidics research is not an easy target. In this article we report several expert-contributions on different topics, so as to provide guidance for young scientists. At the same time we hope that this document will also prove useful for funding institutions and stake holders, to better understand the perspectives and opportunities offered by this research field.
\end{abstract}




\section{Contents}

1. Introduction

\section{Materials and fabrication techniques}

2. 3D optofluidic devices by femtosecond laser micromachining

3. Lithium niobate as an optofluidic platform

4. Silk fibroin films for biophotonic and microfluidic applications

5. Integration of microfluidics with silicon photonic sensors

\section{Imaging}

6. Holographic on-chip microscopy and tomography using lensless computational imaging

7. Live cell imaging with optofluidics

8. Optofluidic microlenses: from tunable focal length to aberration control

\section{Strong light-fluid interaction}

9. Integration of reconfigurable photonics and microfluidics

10. Optofluidic waveguides and resonators

\section{Optics and acoustic forces for cell analysis}

11. High-content physical phenotyping of biological objects with microfluidic dual-beam laser traps

12. Integration of fiber-based tweezers and microfluidic systems

13. Acoustic prefocusing in optofluidic systems

DNA and molecular analysis

14. Optofluidics for single-cell protein analysis

15. Optofluidics for DNA analysis

16. Nanoscale optofluidics

17. Optofluidic immunoassays

\section{Promising future applications}

18. Optofluidics and point-of-need diagnostics for precision medicine and global health

19. Optofluidics in energy 


\section{Introduction - Paolo Minzioni}

University of Pavia

\section{Optofluidics: an emerging and promising topic}

The field of Optofluidics is a relatively new one in the scientific panorama. Although the idea of using fluids to control light, e.g. by spinning-mercury mirrors, dates back to the $18^{\text {th }}$ century, no liquid-mirrortelescope was practically realized before the end of the $20^{\text {th }}$ century. In particular it is only during the last 15 years that the term "optofluidics" has attracted a significant attention, and that many groups have started devoting their research efforts to this field.

The "young age" of this research field is demonstrated by the fact that the first works indexed on the Web of Science database and containing the word "optofluidics" in the topic appears in 2005 [1,2]. Since that moment the attention devoted to the realization of systems exploiting the simultaneous control of fluidic conditions and optical beams roared. In the following twelve years (2005-2016), the number of papers that can be found using the same criteria increases from 0 to 580. Recently about 70 new papers with the word "optofluidics" in the title are published every year, and the number of citations paid to the "optofluidics" paper is continuously growing, almost linearly, and passed from 20 in 2006 to about 1600 in 2016.

Nowadays, what is generally included in the field of Optofluidics is, in the vast majority, based on the integration of optical technologies (for sensing, actuation or imaging) within micro-fluidic (or nanofluidic) systems allowing the use of small samples volumes in a highly-controlled environment. As a result it is also observed that an increasing number of short-schools for Ph.D. students are being scheduled by universities, and topical conferences expressly dedicated to the field of Optofluidics are being organized by important scientific associations (e.g. the EOS Conference on Optofluidics in Europe, the International Multidisciplinary Conference on Optofluidics in Asia, etc.), so as to create meetingpoints for researchers with different backgrounds and interests [3-5].

It is worth underlining that the field of Optofluidics is twice interdisciplinary: not only because of the competences required for the design and realization of the devices, but also because of the countless possibilities offered by these devices in the fields of chemical sensing, physical characterization and biomedical research.

\section{Intended Audience and Aim of the Roadmap}

The intended audience of this document is constituted by three different categories of possible readers: young researchers, scientists from other disciplines, and optofluidics experts.

Young researchers, starting their activity in optofluidics could significantly benefit from this article, as it represents a reference text, showing possibilities and challenges of this specific research field. Additionally the Roadmap also comes with a substantial bibliography making it easier to identify and access many helpful papers.

The Roadmap can be useful as well for researchers who have already developed a good expertise in a contiguous field, such as chemical sensing, microfluidic devices, high sensitivity molecule sensors, single-cell analysis, material-science and highprecision diagnostics. For all of them optofluidics can surely open new possibilities and scenarios, that this Roadmap can help to unveil.

Finally, for experienced researchers in the optofluidic field, the contributions included in this text by wellknown experts allow having a clear reference point about the current state of the art. The Roadmap offers them the possibility to get recent updates on the scientific activities and to analyze what other experienced researchers see as future perspectives.

The aim of this document is that of showing not only the current technologies and applications but also showing promising approaches, still requiring research and technological development. It is in any case perfectly clear to the authors of this paper that what is now perceived as a challenge may, in such a rapidly evolving field, already be achieved in one year, or even less, or may not be considered any more as a relevant target. Nevertheless, it is of great importance to define which research directions are now considered as the emerging and promising, so that it will be possible in the future to re-assess the validity of this analysis.

Another fundamental point that this Roadmap wants to stress, thanks to the different contributions, is the strong connections linking applications, materials, and methods. Exactly for this reason contributions on all these aspects are included in the document. Additionally, even if many topics are discussed in each section, the order of the different contributions has been selected to allow an easier sequential reading.

\section{Current Status and Challenges}

This section wants to briefly provide young researchers, and all those needing some introduction to the basics of optofluidics, with a short list of the main concepts useful to fully exploit the Roadmap content, and a list of suggested introductory readings.

One of the basic ideas in optofluidics is that of taking advantage (at least in the majority of the cases) of the laminar-flow regime that is obtained as a consequence 
of the typical dimensions and flow-speed used in microfluidic systems. The strong laminarity of these systems is testified by their Reynolds number, defined by the below equation, where $\rho$ is the fluid density $\left(\approx 10^{3} \mathrm{~kg} / \mathrm{m}^{3}\right.$ in case of room-temperature water), $v$ is the fluid speed (generally in the range $10^{-5}-10^{-1} \mathrm{~m} / \mathrm{s}$ ), $D$ is the microchannel diameter (of the order of $10^{-4} \mathrm{~m}$ ) and $\mu$ is the dynamic viscosity (which for water is about $10^{-3}$ Pa s):

$$
\boldsymbol{R} \boldsymbol{e}=\frac{\rho v D}{\boldsymbol{\mu}}
$$

If we compare the maximum $R e$ value achievable with the above reported numbers (10) with the reference value of 3000 , generally considered as a boundary between laminar flow $(R e<2000)$ and turbulent flow ( $R e>4000$ ), it is immediate to understand that unless turbulences are artificially created a laminar flow is produced in these systems [6,7]. The flow laminarity, which is a crucial point when well separated streams are needed, can become an issue when fluid mixing is required. In that case specific structures can be used to break the system laminarity [8,9].

Another fundamental element in the optofluidics field is the good size-matching between biological objects (bacteria, cells, or organelles), microfluidic channels, and the diameter of optical beams in the visible or near-infrared spectral range. All these quantities are generally in the range between $0.1 \mu \mathrm{m}$ and $500 \mu \mathrm{m}$, and, while the size of biological objects is generally a given data, it is often possible to tailor the microchannel width and optical beams diameter in order to obtain the desired values and fluidic/optical effects [10].

Additionally, the forces that can be applied to cells by means of optical beams, to trap, push or deform them, well match those generally involved in biological processes [11-17].

Finally, a basic idea in the optofluidic field is that thanks to the different response exhibited by the samples under analysis to optical or acoustic forces it is also possible to use different actuation mechanisms as "sensing mechanisms", enabling for interesting selection and sorting strategies [18-22].

\section{Some Trends \& Challenges}

Without going now into the specific solutions that can be envisaged, there are three main challenges that future optofluidic devices should try to solve.

The first one is dealing with the sample insertion and collection from a microfluidic system. Although the use of extremely reduced sample volumes brings several advantages it is nevertheless evident that properly managing volumes in the $\mu \mathrm{L}$ range (or smaller) obviously requires precision and attention. Even more challenging is the collection of the output samples in all those situations where sub-populations or cells (or even single cells) need to be extracted preserving their viability and without the risk of losing them in microchip dead-volumes.

A second challenge is that of achieving high performances and sensitivities while using low-cost, or disposable, systems. Such an achievement could allow the diffusion of optofluidic solutions in many environments where lab equipment is not always available.

The third challenge that many microfluidic structures are now trying to solve is that of creating easy-to-use devices. At the state-of-the-art microfluidic devices are generally tested on lab tables, requiring a lot of additional instruments (laser sources, thermal controllers, objectives, micropumps, cameras, etc.) and must be operated by experienced researchers. The integration of all these functionalities in a simple micro-system, would largely help the diffusion of these devices, making them user-friendly and more suitable for point-of-care analysis. In particular a considerable attention has been paid in the last five years to the development of compact and efficient imaging systems, which will also be discussed in the following.

Acknowledgments - I want to thank Professor Ilaria Cristiani for her precious suggestions and Dr Jarlath McKenna for the great help in organizing and coordinating the article.

\section{References}

[1] C. Grillet, P. Domachuck, B. Eggleton, J. CooperWhite, "Optofluidics enables compact tunable interferometer”, Laser Focus World 41 (2), 2005

[2] P. Domachuk, M. Cronin-Golomb, B.J. Eggleton, S. Mutzenich, G. Rosengarten, A. Mitchell, "Application of optical trapping to beam manipulation in optofluidics”, Optics Express 13 (19), 7265-7275, 2005

[3] http://www.nanotech.dtu.dk/uddannelse/polynanosummerschool

[4] http://www.myeos.org/events/wpc2017/eosof

[5] http://www.optofluidics.sg/

[6] O. Reynolds, "An experimental investigation of the circumstances which determine whether the motion of water shall be direct or sinuous, and of the law of resistance in parallel channels", Philosophical Transactions of the Royal Society. 174 935-982, 1883

[7] H. Bruus "Acoustofluidics 1: Governing equations in microfluidics” Lab Chip 11, 3742-3751, 2011 
[8] C.Y. Lee, W.T. Wang, C.C. Liu, Lung-Ming Fu, "Passive mixers in microfluidic systems: A review" Chemical Engineering Journal 288, 146-160, 2016

[9] http://www.elveflow.com/microfluidictutorials/microfluidic-reviews-andtutorials/microfluidic-mixers-short-review/

[10] A.M. Smith, M.C. Mancini, S. Nie, "Bioimaging: Second window for in vivo imaging" Nature Nanotechnology 4 (11), 710-711, 2009.

[11] A.Ashkin, “Acceleration and trapping of particles by radiation pressure” Phys. Rev. Lett. 24, 156-158 1970

[12] A. Ashkin, J.M. Dziezic, J.E. Bjorkholm, S. Chu, "Observation of a single beam gradient force optical trap for dielectric particles” Opt. Lett. 11, 288-290 1986

[13] A. Salandrino, S. Fardad, D.N. Christodoulides, "Generalized Mie theory of optical forces," J. Opt. Soc. Am. B 29, 855-866, 2012

[14] L. Ferrara, E. Baldini, P. Minzioni, F. Bragheri, C. Liberale, E. Di Fabrizio, I. Cristiani "Experimental study of the optical forces exerted by a Gaussian beam within the Rayleigh range”, Journal of Optics, 13, 7, 075712, 2011

[15] M.J. Padgett, J. Molloy, D. McGloin Optical Tweezers: Methods and Applications, CRC Press, 2010

[16] T. Yang, F. Bragheri, P. Minzioni “A comprehensive review of optical stretcher for cell mechanical characterization at single-cell level”, MDPI Micromachines 7, 5, 2016.

[17] J. Guck, R. Ananthakrishnan, H. Mahmood, T.J. Moon, C.C. Cunningham, J. Käs, “The Optical Stretcher: A Novel Laser Tool to Micromanipulate Cells” Biphysical Journal, 81 (2), 767-784, 2001

[18] H. Bruus "Acoustofluidics 2: Perturbation theory and ultrasound resonance modes”, Lab Chip, 12, 2028, 2012

[19] J. Dual, T. Schwarz, “Acoustofluidics 3: Continuum mechanics for ultrasonic particle manipulation” Lab Chip 12, 244-252, 2012

[20] T. Yang, F. Bragheri, G. Nava, I. Chiodi, C. Mondello, R. Osellame, K. Berg-Sørensen, I. Cristiani, P. Minzioni “A comprehensive strategy for the analysis of acoustic compressibility and optical deformability on single cells” Scientific Reports 6, 23946, 2016

[21] J. Shi, X. Mao, D. Ahmed, A. Colletti, T.J. Huang "Focusing microparticles in a microfluidic channel with standing surface acoustic waves (SSAW)” Lab Chip 8, 221-223, 2008

[22] L.Y. Yeo, J.R. Friend, "Surface Acoustic Wave Microfluidics”, Annual Review of Fluid Mechanics Vol.46:379-406, Annual Reviews (2014) 


\section{3D optofluidic devices by femtosecond laser micromachining - Roberto Osellame Istituto di Fotonica e Nanotecnologie - CNR}

\section{Relevance of the topic}

Femtosecond laser micromachining (FLM) of transparent materials $[1,2]$ is a recent technique that found wide application in optofluidic device fabrication. FLM is based on a nonlinear absorption process, selectively triggered in the focal volume by ultrashort and tightly focused laser pulses (Fig. 1). Tailoring of the irradiation parameters can produce very different results in glass. In particular, one can achieve a gentle and localized change of the refractive index or a self-assembled nanostructuring of the material. The first modification is extremely relevant as it allows drawing optical waveguides by suitably moving the glass substrate with respect to the laser focus. The second modification is also important for optofluidic applications since the nanostructured region has an enhanced selectivity to wet chemical etching (e.g., acqueous solutions of hydrofluoric acid). This means that an irradiated volume in this second regime will be preferentially etched away with respect to the unirradiated material, thus creating cavities and microfluidic networks inside the glass.

The first relevant advantage of this microfabrication technology for optofluidics is the capability to produce, with the same tool, both optical waveguides and microfluidic channels in glass. This puts the technology in a very favorable condition to combine these two elements in the same substrate and produce compact and complex optofluidic devices. The second advantage of this technology is its unique capability to produce 3D structures taking advantage of the fact that the induced modification is highly localized to the focal volume and can thus be arbitrarily placed anywhere in the glass volume. A third advantage is the possibility to combine very different fabrication techniques with a single tool, from material property tuning (e.g. in waveguide writing), to selective material removal (e.g. for microchannel fabrication) and additive manufacturing by two-photon polymerization (e.g. for the creation of plastic microstructures embedded in the microfluidic channels [3,4]). A final important advantage of FLM is the rapid prototyping capability; as any maskless and direct-write technique, a software design can be transferred forthwith onto a working prototype, with the possibility to simply and rapidly adjust the design or to produce highly customized products.

Further advances in this technology will provide an unprecedented level of integration of functionalities in a single microsystem, with very short time-to-market of new products.

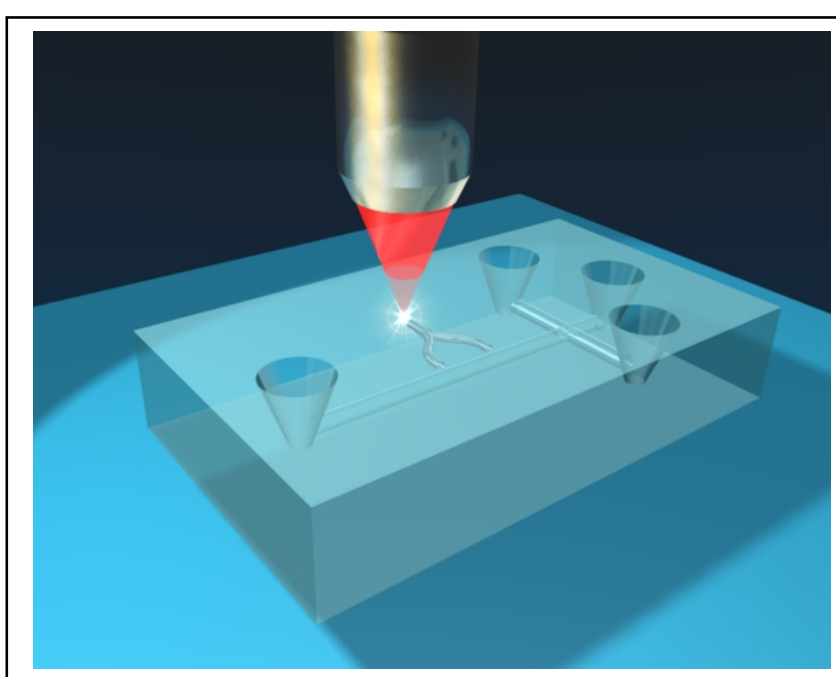

Figure 1. Femtosecond laser micromachining can create optofluidic devices, encompassing optical waveguides and microfluidic channels, directly buried inside a glass sample.

\section{Current Status and Challenges}

FLM has been widely exploited in the fabrication of optofluidic devices with the main aim of integrating optical detection and microfluidic handling of samples [2]. The use of optical waveguides to deliver the probing light and to collect the output signal enables higher sensitivity, reproducible and alignment-free measurements, and multipoint sensing. Taking advantage of the 3D capabilities of the technology, it has been possible either to add specific components to already existing devices or to fabricate the complete microsystem by FLM. An example of the former case consists in adding optical waveguides to commercial microfluidic lab-on-chips to perform on-chip fluorescence or label-free detection of relevant biomolecules [2]. A different example of how FLM can be exploited to add specific components to already existing devices is the concept of Lab-in-Fiber [5]. In this case, the starting device is an optical fiber, where the cladding is typically underused. In this approach, FLM is exploited to add microchannels, cavities or additional optical waveguides to produce highly functionalized optical fibers that become selfconsistent and sophisticated optofluidic sensors (Fig. 2a). The possibility to use FLM to fabricate the whole device further widens the design possibilities and fully unleashes the 3D capabilities of the technology. 3D microfluidic layouts have been exploited to achieve full-hydrodynamic-focusing and, combined with laser written optical waveguides, enabled the demonstration of a $1 \mathrm{~mm}^{3}$ cell counter, capable of counting up to 5000 cells/s [6]. Cell manipulation is another relevant application of FLM-produced devices (Fig. 2b). In fact, optical forces, exerted by the light coming from optical waveguides, can be exploited to trap and deform cells flowing in a microchannel, thus characterizing their mechanical response [7]. This analysis can 
discriminate between different cells populations (e.g., between healthy and cancer cells) without any marker (see Section 11 for more details). Depending on the assay result, each cell can then be sorted in different output channels, again exploiting optical forces produced by a dedicated waveguide. The combination of 3D microfluidics and optical waveguides has been also exploited to produce a 3D-shaped nanoacquarium, to study the behavior of cyanobacteria in controlled conditions [8]. In particular, optical waveguides embedded in the chip were used to accurately monitor the $\mathrm{CO}_{2}$ concentration in the nanoacquarium. Another 3D optofluidic device that allows sophisticated fluorescence imaging of biological samples is the recent demonstration of selective plane illumination microscopy (SPIM) on-chip [9]. An optofluidic

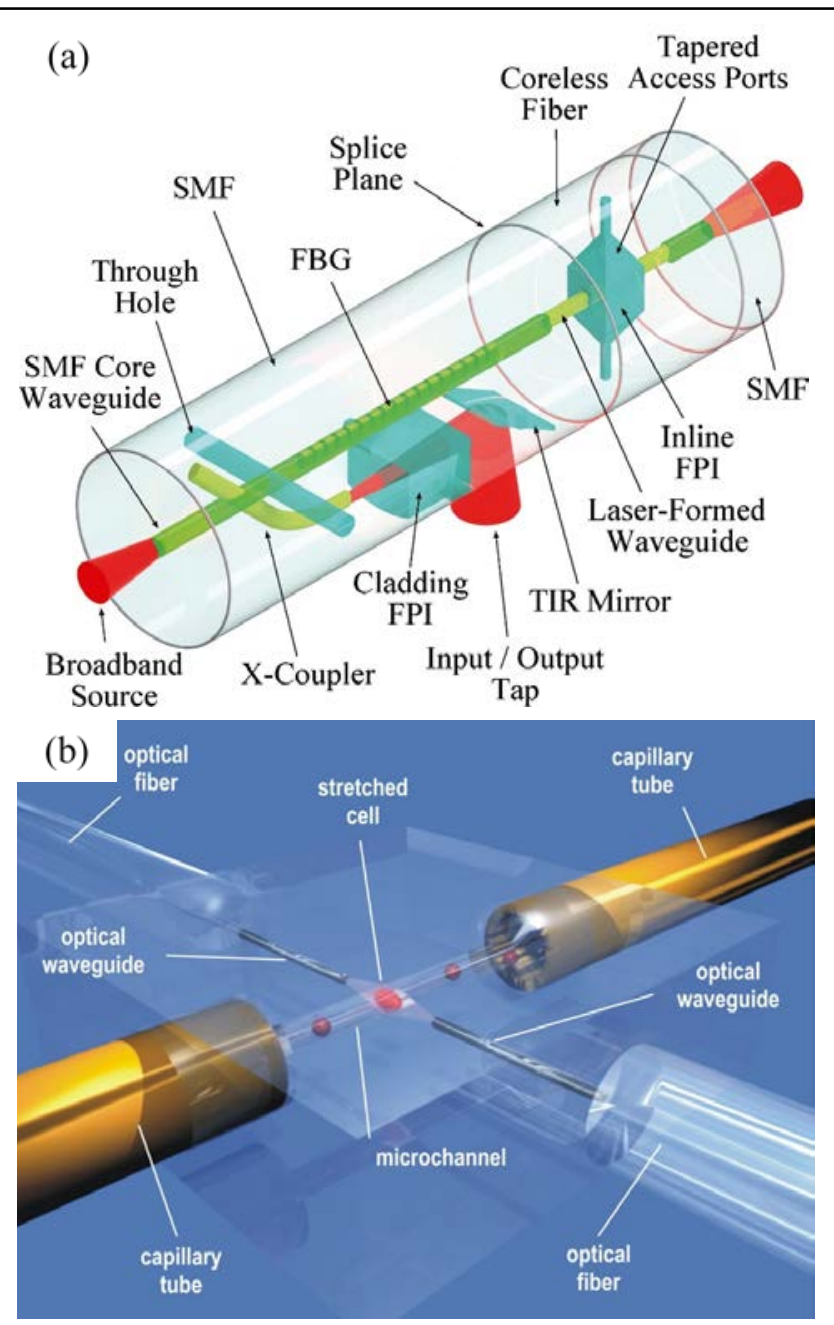

Figure 2. Two examples of optofluidic devices fabricated by femtosecond laser micromachining. (a) Lab-in-fiber: Several optical and fluidic components can be integrated in the cladding of a fiber to perform optofluidic sensing; SMF: single mode fiber; FBG: fiber Bragg grating; FPI: Fabry-Perot interferometer; TIR: total internal reflection. Reproduced from [5] with permission of The Royal Society of Chemistry. (b) Optical cell stretcher: optical forces exerted by light delivered by two optical waveguides allow characterizing the mechanical properties of flowing cells [7]. Reproduced with permission (C) 2010 Optical Society of America. cylindrical lens has been combined with a suitably shaped microchannel, allowing optical sectioning and 3D reconstruction of cellular spheroids in suspension. The biological sample is driven through the light sheet, created by the cylindrical lens, with a uniform microfluidic flow.

As already mentioned, additional functionalities can be integrated in the optofluidic device by two-photon polymerization (2PP). In particular, the 3D capability of FLM enables the 2PP-fabrication of plastic structures directly inside sealed microfluidic channels [3], with an approach that has been dubbed 'ship in a bottle' [4]. As an example, arrays of diffractive and refractive microlenses have been produced inside FLM-fabricated microfluidic channels to achieve white-light detection and counting of cells flowing in the microchannel [4].

A few technological challenges are still open to fully exploit this technology. The first one is FLM throughput; in fact, being a serial writing technique, it requires the time to irradiate each device and does not benefit from economy of scale. A second challenge is the possibility to process different materials, other than fused silica and Foturan glass. The third challenge is further miniaturization to the nanoscale.

\section{Advances in Science and Technology to Meet Challenges}

The challenges previously detailed have triggered a significant effort in the community and, although still open, there are already encouraging results towards their solution. Regarding the scaling of fabrication capability, fine optimization of the processing window has already allowed writing velocities in the order of $\mathrm{cm} / \mathrm{s}$ for several processes. In addition, the use of spatial light modulators has introduced the possibility of multifoci writing, thus enabling the production of several structures in parallel. These approaches have still to be exploited fully to understand the ultimate limits, but FLM will likely be competitive with standard microfabrication technologies also for large production batches and not just for a few prototypes. Fused silica or Foturan glasses are excellent substrates for many optofluidic applications and in particular for biophotonic ones. However, enlarging the portfolio of materials amenable to FLM will be beneficial to target new optofluidic applications. A better understanding of the nanostructuring process, at the base of the microchannel formation, and its replication in other materials is an on-going activity that still requires some effort. Finally, further miniaturization to the nanoscale could be the next big evolution of the technology. The 2PP technique is already able to produce nanoscale structures and recently nanofluidic channels have been demonstrated by FLM [8]. This fabrication scale is still 
largely unexplored, however FLM has the unique capability of easily connecting nanoscale structures to microscale ones, thus potentially improving the usability of new devices at this very small scale.

\section{Concluding Remarks and Perspectives}

Femtosecond laser micromachining is a very powerful technique for the fabrication of optofluidic devices, allowing unique 3D layouts and an amazing level of integration of different functionalities. Its versatility opens the way to many different applications both for research and for industry. In particular, in those fields where standardization is not too rigid, the design freedom allowed by FLM can produce revolutionary microsystems that will set a new standard in the field.

\section{References}

[1] Gattass RR and Mazur E 2008 Femtosecond laser micromachining in transparent materials Nature Photonics 2 219-225

[2] Osellame R, Hoekstra HJWM, Cerullo G and Pollnau M 2011 Femtosecond laser microstructuring: an enabling tool for optofluidic lab-on-chips Laser Photonics Rev. 5 442-463

[3] Amato L, Gu Y, Bellini N, Eaton SM, Cerullo G and Osellame R 2012 Integrated three-dimensional filter separates nanoscale from microscale elements in a microfluidic chip Lab Chip 12 1135-1142

[4] Wu D, Xu J, Niu L-G, Wu S-Z, Midorikawa K and Sugioka K 2015 In-channel integration of designable microoptical devices using flat scaffoldsupported femtosecond-laser microfabrication for coupling-free optofluidic cell counting Light: Science \& Applications 4 e228

[5] Haque M, Lee KKC, Ho S, Fernandes LA and Herman PR 2014 Chemical-assisted femtosecond laser writing of lab-in-fibers Lab Chip 14 38173829

[6] Paiè $P$, Bragheri $F$, Martinez Vazquez $R$ and Osellame R 2014 Straightforward 3D hydrodynamic focusing in femtosecond laser fabricated microfluidic channels Lab Chip 14 1826-1833

[7] Bellini N, Vishnubhatla KC, Bragheri F, Ferrara L, Minzioni P, Ramponi R, Cristiani I and Osellame R 2010 Femtosecond laser fabricated monolithic chip for optical trapping and stretching of single cells Opt. Express 18 4679-4688

[8] Sugioka K, Xu J, Wu D, Hanada Y, Wang Z, Cheng Y and Midorikawa K 2014 Femtosecond laser 3D micromachining: a powerful tool for the fabrication of microfluidic, optofluidic, and electrofluidic devices based on glass Lab Chip 14 3447-3458

[9] Paiè P, Bragheri F, Bassi A and Osellame R 2016 Selective plane illumination microscopy on a chip Lab Chip 16 1556-1560
[10] Hasegawa S, Ito H, Toyoda H and Hayasaki Y 2016 Massively parallel femtosecond laser processing, Opt. Express 24 18513-18524 


\section{Lithium niobate as an optofluidic platform - Cinzia Sada \\ University of Padova, Italy \\ Relevance of the topic}

Droplet-microfluidics technology has paved the way to new approaches of chemical and biological analysis, exploiting parallel processing and high throughput response. Screening, rapid droplet sorting and biochemical micro-reactors were in fact demonstrated as well as bio-analytical assays, enzyme kinetics, cell analysis, encapsulation and sorting. Thanks to the exploitation of reduced volumes down to nano-litres to femto-litres, as well as reaction times shortened to seconds or less, chemical synthesis and complex particle fabrication up to drug delivery and diagnostic testing and biosensing were also reported [1]. Although novel micro-fabrication techniques are continuously being developed to improve the final performances, lower the devices costs and differentiate functionalities with appropriate fluidic interfacing scheme, fully integrated opto-microfluidics devices are far from being widely commercially available yet. The perspective of combining the optical tools versatility with the potentiality of microfluidics has gained increasing interest. As already foreseen by Psaltis and co-workers in their pioneering work published in Nature on 2006, novel enabling technologies leading to true Lab-on-chip system have started to be under debate where chemical, physical and biophysical sensors [2] could be integrated on multi-purpose portable devices. To this aim, several challenges should be faced both from a scientific and a technological point of view. An opto-microfluidic portable device should in fact guarantee a trustworthy response, i.e., a reproducible and repeatable output that, minimizing false responses and artefacts, is easy to read/store and fast to calibrate. Finally, to be really competitive with full-optional bulky-lab apparatuses, it should be compact, low cost, easy to use and handle, and stable under different environmental conditions. At first sight, all these features seem difficult to match. The demand of reducing costs has therefore prevailed, leading towards the spread of disposable devices and putting in second priority the possibility of checking, monitoring and exploiting the device response in a longer time scale. However in the last decade, draining the expertise in photonics, integrated optics and microelectronics MEMS processing into microfluidics, have conveyed advances to overcome and bypass these limitations by the suitable combination of materials, preparation techniques and smart approaches respectively [3].

\section{Current Status and Challenges}

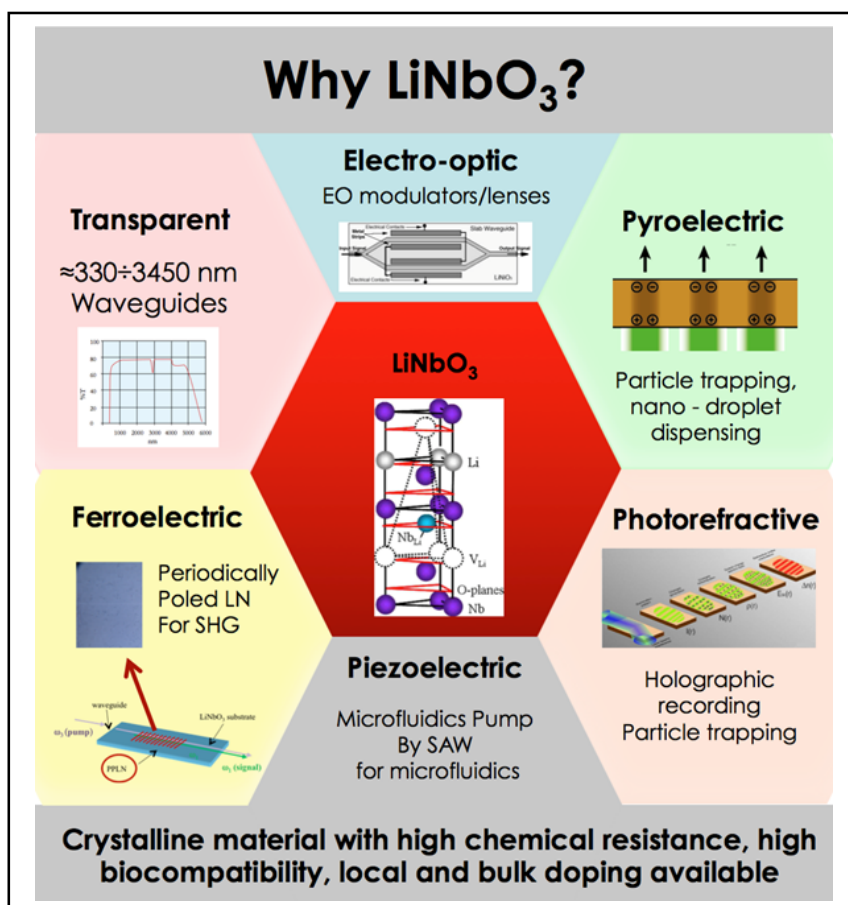

Figure 1 - Lithium niobate $\left(\mathrm{LiNbO}_{3}\right)$ main properties.

In lab-on-chip systems the integration of many stages is a key point in portable compact devices. Consequently, flexible and easily-moulded materials (mainly polymers such as PDMS, PMMA) and biocompatible substrates such as glass and silica have usually been used leading to commercially available disposable microfluidic systems. Opto-fluidics platforms were therefore achieved by coupling both laser beams (including, in best cases, fibres coupling) and detection stages (often carried out by optical microscopy) to microfluidics. To this direction, prototypes able to detect, count, sort and analyse the droplets have therefore been investigated such as: compact micro-flow-cytometers (typical throughput of the order of several hundreds of cells per second and a typical sample purity about $90 \%$ ), velocity detectors [4], biosensors based on fluorescence measures [5] just to cite a few. In these cases, frequently polymers were bonded to rigid materials: silicon, to provide for patterned substrate to anchor/align fibres to the microfluidic circuitries; silica or glasses to provide for a solid base; lithium niobate (LN) to add Surface Acoustic Wave (SAW) stages working as micro-pumps or mixers respectively. Quite surprisingly, only in the last decade significant advances were achieved by delivering monolithic, perfectly aligned, robust and portable opto-fluidic prototypes. Both microfluidic circuits and optical waveguides were synergistically fabricated on the same substrate (fused silica) by femtosecond laser micromachining (FLM) [6]. Optically controlled photonic prototypes to manipulate micron-scale dielectric objects by light-driven phenomena were therefore presented: optical tweezers and stretchers were implemented with suitable focused 
laser beams, either externally or fibre-coupled, at a microfluidics level [7]. Similar results can be achieved also in LN crystals substrates but are currently underestimated (Fig.1). Thanks to its excellent optical and electro-optical properties, in fact, high quality optical waveguides, multiplexers, switches and optical modulators are commercially available, therein obtained mainly by Ti-in-diffusion technology. Its ferroelectric property, instead, was exploited to produce light sources by way of frequency conversion in Periodically Poled LN structures. Other features, instead, were less profit by: biocompatibility, photorefractivity for holographic recording and induced local space-charge-fields to exploit dielectrophoretic and electrophoretic forces (either generated by inhomogeneous illumination [8] or heating [9]) as well as micromachining by high optical quality dicing [10]. This material therefore presents all the needed characteristics to host several different functionalities on the same substrate in a monolithic integrated opto-fluidic multi-functional platform. This high level of integration represents a great challenge but is required in portable Lab-on-chip systems that could operate in a wide spectrum range, with tailored final response and real-time reconfigurability. Limiting factors mainly stem from either the tools to finely control the fluids flux and the optimization of the light coupling and detection within the microfluidics interface. Up to now expensive and bulky systems such as syringe or pressure based pumps are still used whilst optical functionalities are often granted by using high power or optical bench working lasers respectively. When optical fibres are integrated, instead, optical losses can represent a limiting factor for enhanced sensibilities. Hybrid configurations of polymers/ durable materials were therefore proposed to partially overcome this issue. Less investigated, instead, were the alternative solution of monolithically integration of several functionality on the same substrate. Both these aspects somehow have hindered the full exploitation of opto-microfluidics benefits and potentialities so far.

\section{Advances in Science and Technology to Meet Challenges}

In order to get a true portable device some issues must be solved from a technological point of view. In integrated opto-microfluidics platforms, embedded or buried optical waveguides are in fact needed. Up to now only the FLM technique allowed one to reach such a result, but in fused silica and in a defined range of possible functionalities limited to this materials properties. Although some attempts were made in $\mathrm{LiNbO}_{3}, \mathrm{FLM}$ is still far from being truly exploited. As an alternative, LN could be also micromachined and bonded to silica and to polymers, allowing hybrid configurations as well. Less hunted, instead, seems the

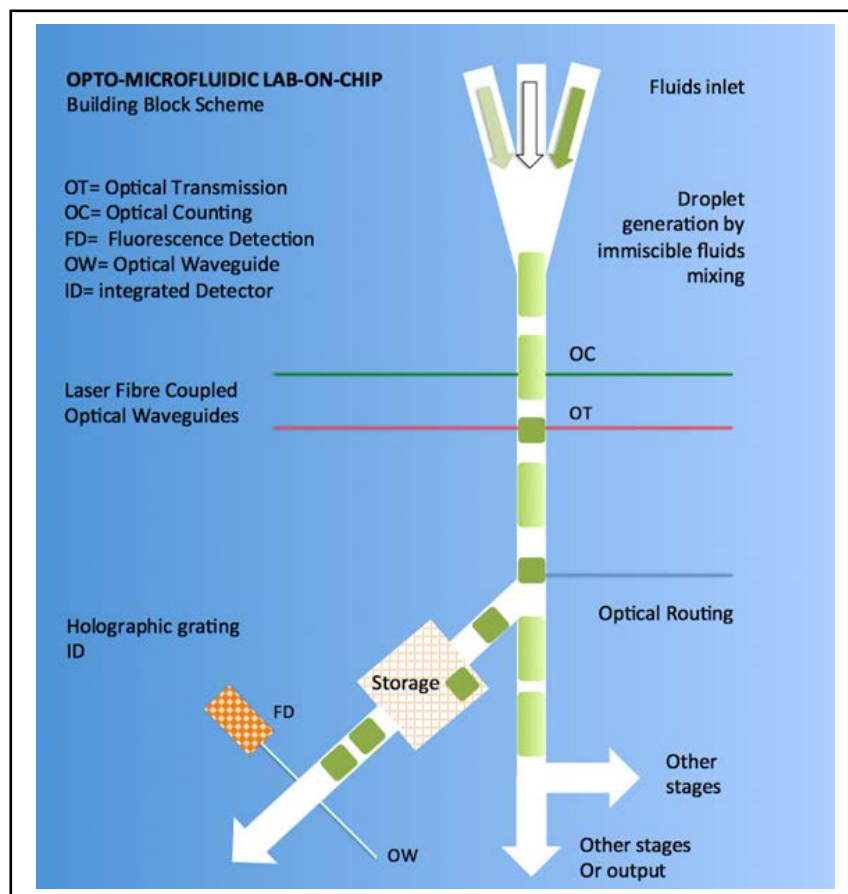

Figure 2 - Lithium niobate based Opto-fluidic platform.

$\mathrm{LiNbO}_{3}-\mathrm{LiNbO}_{3}$ bonding to get 3D configurations, still almost unexplored. Despite of these technological challenges, the real advance in science is straightforward: droplet counting, sorting depending on the size or content, droplet routing and storage allintegrated on the same substrate with micro-pumps fluid driving can be foreseen. This is possible by implementing droplets optical transmission measurements and passage monitoring as well as refractive index and/or fluorescence emission detection (eventually spectral resolved by recording holographic gratings). Finally, waveguides can both confine light and allow for droplet routing and sorting, while storage can be achieved (Fig.2). This challenge can be faced only by exploiting light-induced phenomena in the material, something possible in lithium niobate by way of its photorefractive and photovoltaic properties, even enhanced by suitable local doping. Consequently tailored and reconfigurable set-ups could be designed even in real-time by way of feedback systems triggered by the optical waveguides response as well.

\section{Concluding Remarks and Perspectives}

Light-reconfigurable devices with multifunctional stages have not been proposed yet on the same substrate, as well as systems that activate a function in real time depending on the other stages feedback. Together with the miniaturization advantages, a fully integrated opto-fluidic platform promises to provide higher sensitivity and dynamic range, a finer control of the light power focused on the biological target and a dynamical reconfigurability tailored depending on the final purpose. Integrated optics can provide a perspective and a systematic analysis on the role of 
different conditions of illumination and the relative real-time monitoring even of cumulative light induced effects still unknown or not investigated. Lithium niobate promises to be a valid alternative, as it was demonstrated to host: efficient integrated optics stages; micro-pumps by Surface Acoustic Waves (SAW); SHG light generation by PPLN; particle manipulation stages and droplet routing by way of photovoltaic properties; engraved microfluidic circuitries by Femtolaser technique and high quality dicing. Consequently 1. it represents an ideal gym-floor to integrate all these 2 . functionalities together and achieve a portable full optional Lab-on-chip device, tailored and reconfigurable by way of optical induced phenomena.

Acknowledgments - The author kindly acknowledge G. Bettella, G. Pozza, C. Montevecchi, E. Baggio, R. Zamboni and A. Zaltron, co-workers at University Padova. Fondazione Ca.Ri.Pa.Ro and the University of Padova are acknowledged for funding the Excellence Project-2012 and PRAT-2016 project respectively.

\section{References}

[1] Seeman R, Brinkmann M, Pfohl T, Herminghaus S 2012 Rep. Prog. Phys. 75 016601-42

[2] Matos Pires NM, Dong T, Hanke U, Hoivik N 2014 Recent Developments in Optical Detection Technologies in Lab-on-a-Chip Devices for Biosensing Applications Sensors 14(8) 1545815479

[3] Pang L, Chen MH, Freeman LM, Fainman Y 2012 Optofluidic devices and applications in photonics, sensing and imaging Lab on a Chip 12(19):3543-51

[4] Hsieh YW, Wang AB, Lu XY, Wang LA 2016 High-throughput online multidetection for refractive index, velocity, size and concentration measurements of micro-two-phase flow using optical microfibers Sensors and Actuators B 237 841-848

[5] Testa G, Persichetti G, Sarro PM, Bernini R 2014 A Hybrid silicon-PDMS optofluidic Platform for sensing application Biomedical Optics Express 14 (2) 417-426

[6] Osellame R, Hoekstra HJWM, Cerullo G, Pollnau M 2011 Femtosecond laser microstructuring: an enabling tool for optofluidic lab-on-chips Laser \& Photon Rev 5 442-463

[7] Bragheri F, Ferrara L, Bellini N, Vishnubhatla KC, Minzioni P, Ramponi R, Osellame R, Cristiani C 2010 Optofluidic chip for single cell trapping and stretching fabricated by a femtosecond laser Journal of Biophotonics 3 (4) 234-243

[8] Esseling M. Zaltron A, Horn W, Denz C, Optofluidic droplet router 2015 Laser and Photinics reviews 9 (1) 98-104
[9] Ferraro P, Coppola S, Grilli S, PAturzo M, Vespini V 2010 Dispensing nano-pico droplets and liquid patterning by pyro-electrodynamic shooting Nature Nanophotonics 5 429-435

[10] Chauvet M, Al Fares L, Guichardaz B, Devaux F, Ballandras S 2012 Integrated optofluidic index sensor based on self-trapped beams in LiNbO3 Applied Physics Letters 101 (18) 181104-6 
4. Silk fibroin films for biophotonic and microfluidic applications - S. Zhao, F. G. Omenetto

Tufts University

\section{Relevance of the topic}

Microfluidics is a versatile technological platform for precise manipulation of fluids of extremely small volumes and has a broad range of biochemical and medical applications. Polydimethylsiloxane (PDMS), as one of the most commonly used microfluidic materials, has revolutionized the field by enabling outof-cleanroom and inexpensive fabrication and providing robust material properties to support a variety of applications from large-scale chemical synthesis to high throughput bimolecular assays. However, the non-biofriendly fabrication process and the limitation on bulk functionalization have limited the utility of PDMS devices in the fields of tissue engineering and regenerative medicine. More recently, biologically relevant materials have been utilized to construct microfluidic devices, such as gelatin, collagen, alginate and silk. These natural biomaterials allow easy bulk and surface functionalization and exhibit fully compatible bio-interfaces. Among those materials, silk fibroin has unique physicochemical properties including highly tunable mechanical stiffness and in vivo degradability as well as long term stability in various medium environments. These attributes are essentially important for advanced applications of microfluidics in tissue-related research and in vivo implantation.

\section{Current Status and Challenges}

The first microfluidic device completely made of silk fibroin material was introduced in 2007 [1]. The microchannels were fabricated by aqueous casting of silk fibroin solution on PDMS mold followed by water-stable treatment. The micromolded silk film was then laminated onto a flat silk film so closed microchannels were formed. Human hepatocytes were successfully cultured in the microchannels and perfused with cell culture medium for a prolonged period of time to demonstrate the biocompatibility of the devices. A new enzymatically crosslinked silk hydrogel material was later introduced and has also been utilized for microfluidics fabrication (Figure 1). This material provides better tunability on mechanical properties to match different human tissues, bulk functionalization and cell encapsulation for artificial tissue/organ constructions [2]. A gelatin sacrificial molding method was used to create microchannels in silk hydrogel and a layer-by-layer assembly approach allowed the fabrication of multilayered 3D microfluidic devices. Pneumatic fluidic controls previously used in the PDMS counterparts have also

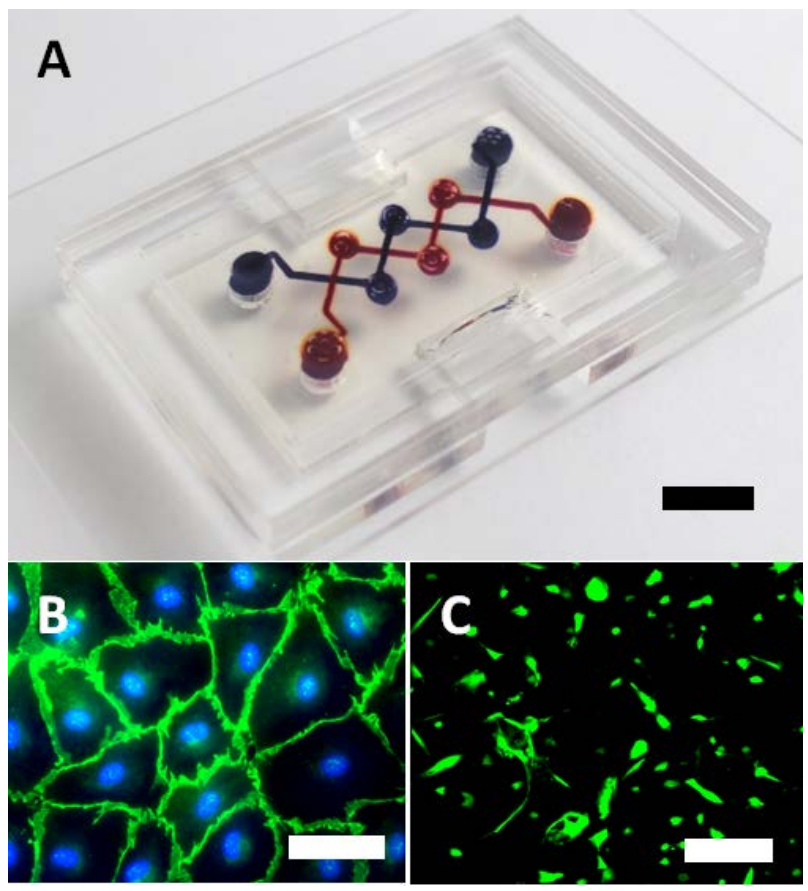

Figure 1. (A) A silk hydrogel microfluidic device with 3D microchannels. (B) Human endothelial cells cultured along microchannel surface. (C) Human fibroblast cells cultured in the bulk of silk hydrogel device. Scale bar shows $1 \mathrm{~cm}$ in (A) and $20 \mu \mathrm{m}$ in (B) and (C). Reprinted with permission from Elsevier [2].

been implemented thanks to the elastic nature of the silk hydrogel, which would allow automatic control of silk microfluidics for high throughput large scale devices. Human endothelial cells and fibroblast cells were successfully cultured along the channel surface and in the bulk of the device, respectively, which demonstrated the utility of the silk hydrogel devices in potential tissue engineering applications.

Silk microfluidics has made tremendous progress in the last decade, but challenges still remain to be addressed to bring this platform from research labs to hospitals and clinical market. One of the key challenges is that the current 3D silk microfluidic fabrication requires time-consuming layer-by-layer assembly and significant human intervention, which largely limits the throughput. Ideally, complex and multilayer silk microfluidics will be fabricated with automatic systems using either bottom-up (e.g. 3D printing) or top-down (e.g. 3D laser micromachining) approaches. Moreover, fabricating ultrafine features (from micrometer to nanometer) within the silk hydrogel is very challenging, which if addressed could allow the construction of high density microvascular networks and highly integrated microfluidic devices.

\section{Advances in Science and Technology to Meet Challenges}

3D printing is a promising technology that allows facile fabrication of complex 3D structures with minimal human intervention. We have recently 
developed a 3D bio-printer that is able to print roomtemperature curable silk hydrogels into various 2D and 3D formats [3]. Ultrafast laser pulses of relatively low energy have also been utilized to directly carve high resolution channels of micrometer dimension within bulk silk hydrogels, which provides a promising solution to automatic fabrication of high density silk microfluidics [4]. In addition to enzyme-induced crosslinking, light and electron beams have also been utilized to cure and pattern silk hydrogel materials under aqueous and bio-friendly conditions, which could be potentially used to fabricate features of micrometer to nanometer resolution $[5,6]$.

\section{Concluding Remarks and Perspectives}

The next evolution of the field of microfluidics will be based on the integration of new classes of active materials as the bulk constituent of the devices. That is, the availability of materials that are biologically active and that can be integrated into living tissue. Together with the possibility of incorporating dopant molecules, which can surround the volumes of fluid flowing through microchannels, with active environments that can define diffusive profiles into or out of the channnels or can provide externally addressable functions. Future applications envisioned are biologically integrated fluidic devices as smart tissue engineering platforms, active bulk doped microfluidics that embed optical and/or electronic function, and dynamically reconfigurable, chemically active devices, extending the utility of the platform.

\section{Acknowledgments}

The authors would like to acknowledge support from the ONR, AFOSR, and NIH.

\section{References}

[1] Bettinger CJ, Cyr KM, Matsumoto A, Langer R, Borenstein JT and Kaplan DL, 2007, Fibroin Microfluidic Devices, Advanced Materials, 19(5): 2847-2850

[2] Zhao S, Chen Y, Partlow BP, Golding AS, Tseng P, Coburn J, Applegate MB, Moreau JE, Omenetto FG, Kaplan DL. 2016, Bio-functionalized silk hydrogel microfluidic systems. Biomaterials, 93:60-70

[3] Jose RR, Brown JE, Polido L, Omenetto FG, Kaplan DL, 2015, Polyol-Silk Bioink Formulations as TwoPart Room-Temperature Curable Materials for 3D Printing, ACS Biomater. Sci. Eng., 1 (9), 780-788

[4] Applegate MA, Coburn J, Partlow BP, Moreau JE, Mondia JP, Marelli B, Kaplan DL, and Omenetto FG, 2015, Laser-based three-dimensional multiscale micropatterning of biocompatible hydrogels for customized tissue engineering scaffolds,
Proceedings of the National Academy of Sciences of the United States of America, 112(39), 12052-12057

[5] Applegate MA, Partlow B, Coburn J, Marelli B, Pirie, Pineda R, Kaplan DL, and Omenetto FG, 2016, Photocrosslinking of Silk Fibroin Using Riboflavin for Ocular Prostheses. Advanced Materials, 28(12), 2417-2420

[6] Kim S, Marelli B, Brenckle MA, Mitropoulos AN, Gil ES, Tsioris K, Tao H, Kaplan DL, and Omenetto FG, 2014, All-water-based electron-beam lithography using silk as a resist, Nature Nanotechnology, 9(4): p. 306-310 


\section{Integration of microfluidics with silicon photonic sensors - Kristinn B. Gylfason and Tommy Haraldsson KTH Royal Institute of Technology}

\section{Relevance of the topic}

With our rapidly advancing understanding of molecular biology, the interest in analysing the molecular composition of various biological samples has increased dramatically in recent years. Medical diagnostics are, for example, increasingly based on the quantitative measurement of the concentration of certain biomolecules, referred to as biomarkers, in patient samples such as blood and urine. Currently, this type of analysis is predominately performed in a laboratory setting, due to the stringent environmental control and the high skill level of operators required for reliable results. However, there is a growing need for reliable mobile bioanalysis, e.g. to tackle epidemics and biohazard monitoring on site.

The great success of current bioanalytical techniques is largely built on the exploitation of highly selective binding reactions of certain biomolecule pairs, e.g. certain proteins and nucleic acids, to perform biomolecule detection with high selectivity. In this way, we take advantage of the work already done by evolution on the fine tuning of the control systems of life. Alas, with this benefit comes a strict condition: we need to handle liquid samples, since biological binding reactions invariably take place in aqueous solutions.

Microfluidics refers to the technology of fluid manipulation on the microscale. From its inception almost 30 years ago [1], this field has benefited greatly from the microfabrication techniques of the microelectronics industry, but has recently developed its own toolset based on polymer micro-structuring, often by casting or moulding. The rapid development of microfluidics has been driven by the desire to automate and parallelize liquid sample handling for applications such as medical diagnostics, biological research, and drug development.

The result of a bioanalysis must be quantified, and optical detection is a particularly popular quantification approach, due to its large dynamic range, high spatial and temporal resolution, and strong resistance to electromagnetic interference. Silicon photonics is the study and application of integrated optical systems which use silicon as an optical medium, usually by confining light in optical waveguides etched into the surface of silicon-on-insulator (SOI) wafers. By fixing one part of the biological pairs mentioned above onto the surface of such a waveguide, the selective binding of the other part can be optically detected. The fixed molecule is referred to as a recognition element, the detected molecule as the analyte, and the technique as a whole as optical biosensing. Since the transparency window of silicon lies in the near- and mid-infrared, detection schemes based on refractive index changes upon biomolecule binding are most commonly applied. Fluorescence based detection is uncommon, since very few fluorophores operate in the near- and mid-infrared.

The main benefit of silicon photonics is that by leveraging the investments made by the silicon-based microelectronics industry, silicon photonics can be efficiently manufactured on large diameter wafers using highly automated processes. Furthermore, silicon based optical circuits can be made very compact on the SOI platform, due to the high refractive index contrast, which enables small waveguide bending radii. This permits efficient use of the wafer area, thereby keeping device cost down. For bioanalysis, this means that whole optical detector arrays can be integrated into a single microfluidic channel. Another benefit of silicon photonics is that electronics for readout and control can be integrated into the same substrate, yielding an unprecedented integration density. The main limitation of silicon photonics is that there are currently no monolithically integrated lasers available, but recent advances on germanium lasers on silicon [2] hold great promise for low cost silicon photonic systems in the near future.

By combining the tools of silicon photonics and microfluidics, the basic toolset is in place for implementing robust mobile bioanalysis. A successful merger, however, requires overcoming a number of challenges outlined below.

\section{Current Status and Challenges}

To target mobile bioanalysis, the chosen integration technology should preserve the key benefits of silicon photonics mentioned above. This implies that both recognition element attachment and microfluidics integration should be done at wafer level, and without adding significant wafer footprint. So far, such an approach has not been demonstrated.

The second fundamental challenge is the volumes mismatch. For many important medical conditions, the concentration of the indicative biomarker analytes is very low in the sample liquid. Thus, for reliable detection, a certain minimum sample volume must be analysed. For common cases, this results in sample volumes of 0.5 to $1 \mathrm{ml}$. Furthermore, most biosensing protocols call for washing with at least 5 times the sample volume of clean buffer liquid. For comparison, a silicon photonic chip of $10 \times 10 \mathrm{~mm}^{2}$ area covered with a uniform $0.1 \mathrm{~mm}$ thick liquid film holds only $10 \mu \mathrm{l}$ of liquid. Any microfluidic channel network structured on top of such a chip will hold much less. Hence, a mobile silicon photonics biosensing 
instrument must be able to handle and store much more liquid than can be fitted on the silicon chip surface.

The third fundamental challenge is the sensitivity of the biological recognition elements to temperature and solvents. This is particularly true for the more interesting protein-based class of recognition elements that generally neither tolerate heating significantly above body temperature nor non-physiological solvents, which severely limits fabrication options. Nucleic acids, on the other hand, are more tolerant, but then there already exist many more alternative analysis methods for nucleic acids.

Figure 1 provides a schematic overview of the integration approaches that have been reported so far, as well as a few possibilities that have not been tried yet. The liquid handling methods can be classified into two major groups, based on analyte transport modality, as advective or diffusive. The advective class is dominated by microfluidic channel based devices, usually employing pressure-driven flow in channels moulded into polymers such as polydimethylsiloxane (PDMS) [3,4] and thiol-enes [5], and transfer-bonded onto the silicon photonics. So far, only chip level processing, has been demonstrated. The critical aspect of this approach is how to seal the channels after recognition element attachment, without affecting the biomolecules. PDMS is difficult to dry-bond to silicon after biomolecule attachment, since the required oxygen plasma activation would destroy the biomolecules. Thiol-enes are more promising in this regard [6]. Another approach is to etch channels into a spin-coated perflouropolymer and then seal them with clamped gaskets [7]. The drawback of this approach is that the poor alignment accuracy of the clamped gasket severely limits the integration density. Furthermore, a leak tight seal will be difficult to achieve in a low-cost consumer operated device. Pressure has also been used to flow samples through orifices in silicon photonic chips glued to the bottom of open ended plastic test tubes [8]. Advective droplet transport (digital microfluidics), which employs electro-wetting, has also been reported [9], but the sample volumes that can be handled in this way are very small.

Due to their simplicity, diffusive transport devices are potentially very interesting for mobile biosensing and high throughput screening applications. However, they will struggle with the low analyte concentrations of most medical diagnostic applications.

\section{Advances in Science and Technology to Meet Challenges}

To be able to structure microfluidic circuits directly on top of silicon photonic wafers, some advances in polymer science are needed. The ideal polymer should be patternable using standard photo-lithography, and

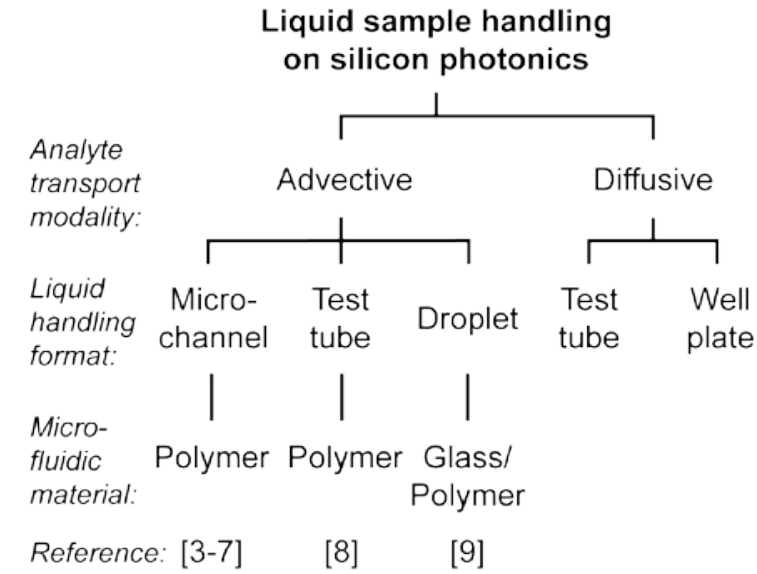

Figure 1 - A schematic overview of the microfluidic integration approaches that have been reported, as well as a few new possibilities.

allow for low-temperature dry bonding both to silicon and to other polymers. Using such a polymer, the alignment-critical first microfluidic channel layer could be made directly on the silicon photonics wafer, using spin coating, lithography, and development. Next, the biological recognition elements could be deposited in a serial process using a spotting robot, or preferably using a wafer-scale printing scheme with a patterned stamp. Then, a second, lower resolution lid layer could be transferred and dry-bonded to form closed channels with open vias aligned to the channel structure below. The lid layer could be injection moulded into a thermoplastic, and thus also contain the large volume reservoirs necessary for ml-scale sample handling. Another alternative would be to add a third layer containing the large liquid reservoirs.

Currently, there exist polymers that have the potential to allow such a processing scheme. In particular, the class of photo-curable thiolene-epoxy polymers [10] seems promising, due to its capability for highresolution photo-patterning and versatile lowtemperature dry bonding.

Another potentially rewarding development of silicon photonics based biosensing would be to use IR fluorescence in combination with silicon photonics. This would enable the combination of the high signal to background possible with fluorescence based sensing with the low-cost, high integration density optics possible with silicon photonics. There are already recent developments on IR fluorophores, but combining them with silicon photonics is uncharted territory.

\section{Concluding Remarks and Perspectives}

Silicon photonics has already been commercially employed to biosensing in a laboratory setting. However, the big potential of the technology is to enable quantitative and reliable mobile bioanalysis, for 
example in a smart-phone attachment. To get there, advances are necessary in wafer-scale polymer processing, as well as in wafer-scale biomolecule attachment. Furthermore, a monolithically integrated light source is also needed to realize this vision. Finally, even though optical sensing, and biosensing in particular, has been the driving force behind much of the recent work, the control and manipulation of light by fluids is a potentially rewarding application of the microfluidic integration technology discussed here.

Acknowledgments - This work was partially supported by the Swedish Research Council (Grants nr. B0460801 and 621-2012-5364).

\section{References}

[1] Manz A, Graber N and Widmer H M 1990 Miniaturized total chemical analysis systems: A novel concept for chemical sensing Sens. Actuators B Chem. 1 244-248

[2] Camacho-Aguilera R E, Cai Y, Patel N, Bessette J T, Romagnoli M, Kimerling L C and Michel J 2012 An electrically pumped germanium laser $O p t$ Express 20 11316-11320

[3] Carlborg C F, Gylfason K B, Kazmierczak A, Dortu F, Polo M J B, Catala A M, Kresbach G M, Sohlström H, Moh T, Vivien L, Popplewell J, Ronan G, Barrios C A, Stemme G and Wijngaart 2010 A packaged optical slot-waveguide ring resonator sensor array for multiplex label-free assays in labs-on-chips Lab Chip 10 281-290

[4] De Vos K, Girones J, Claes T, De Koninck Y, Popelka S, Schacht E, Baets R and Bienstman P 2009 Multiplexed Antibody Detection With an Array of Silicon-on-Insulator Microring Resonators Photonics J. IEEE 1 225-235

[5] Errando-Herranz C, Saharil F, Romero A M, Sandström N, Shafagh R Z, van der Wijngaart W, Haraldsson T and Gylfason K B 2013 Integration of microfluidics with grating coupled silicon photonic sensors by one-step combined photopatterning and molding of OSTE Opt Express 21 21293-21298

[6] Carlborg C F, Cretich M, Haraldsson T, Sola L, Bagnati $\mathrm{M}$, Chiari $\mathrm{M}$ and van der Wijngaart $\mathrm{W}$ 2011 Biosticker: Patterned microfluidic stickers for rapid integration with microarrays 15 th International Conference on Miniaturized Systems for Chemistry and Life Sciences (Seattle, Washington, USA) pp 311-313

[7] Iqbal M, Gleeson M A, Spaugh B, Tybor F, Gunn W G, Hochberg M, Baehr-Jones T, Bailey R C and
Gunn L C 2010 Label-Free Biosensor Arrays Based on Silicon Ring Resonators and High-Speed Optical Scanning Instrumentation IEEE J. Sel. Top. Quantum Electron. 16 654-661

[8] Arce C L, Van Put S, Goes A, Hallynck E, Dubruel P, Komorowska K and Bienstman P 2014 Reaction tubes: A new platform for silicon nanophotonic ring resonator sensors J. Appl. Phys. 115044702+

[9] Lerma Arce C, Witters D, Puers R, Lammertyn J and Bienstman P 2012 Silicon photonic sensors incorporated in a digital microfluidic system. Anal. Bioanal. Chem. 404 2887-2894

[10] Carlborg C F, Vastesson A, Liu Y, van der Wijngaart W, Johansson M and Haraldsson T 2014 Functional off-stoichiometry thiol-ene-epoxy thermosets featuring temporally controlled curing stages via an UV/UV dual cure process J. Polym. Sci. Part Polym. Chem. 52 2604-15 


\section{Holographic on-chip microscopy and tomography using lensless computational imaging - Yibo Zhang and Aydogan Ozcan University of California, Los Angeles}

\section{Relevance of the topic}

The recent advances in micro- and nano-technologies have greatly enhanced our capability to build costeffective and powerful chip-size microsystems that can handle, e.g., cells and bio-fluids. These lab-on-a-chip systems have wide applications in bioengineering and medicine. The integration of optical imaging and microscopy techniques with microfluidics can enable high-throughput detection and sensing of biological and/or chemical signals. To complement such microfluidics and lab-on-a-chip systems, optical microscopy tools need to undergo a transformation in terms of their size, cost and throughput, and computational on-chip imaging provides unique opportunities for this broad goal.

\section{Current Status and Challenges}

The optical interrogation of current microfluidic samples is by and large conducted by conventional light microscopy tools that use lenses for image formation, and have significantly smaller fields of view (FOV) than the size scale of a typical microfluidic chip and shallower depths of field (DOF) than the height of typical microfluidic channels. Moreover, the FOV as well as DOF of conventional microscopy scale down when switched to higher-magnification objective lenses (when higher resolution is needed), further reducing the available imaging area and volume within the sample. Besides, the bulkiness and high cost of these conventional microscopes present another obstacle to merge optical imaging with microfluidics for building lab-on-a-chip systems, especially for use at low-resource and field settings. This mismatch caused by the limitations of traditional optical imaging modalities prompts advances in microscopy.

Meanwhile, tomographic imaging techniques that enable sectional imaging have been of great interest to reveal 3D structures of various organisms. Sectional imaging modalities such as optical projection tomography, optical diffraction tomography, confocal microscopy and light sheet microscopy, among others, can achieve high-resolution 3D optical imaging. However, these modalities are also limited by the aforementioned restrictions including low throughput, high cost and bulkiness.

Therefore, it is of great importance to develop highresolution microscopic and tomographic imaging techniques that have small footprints and large sample imaging areas/volumes.

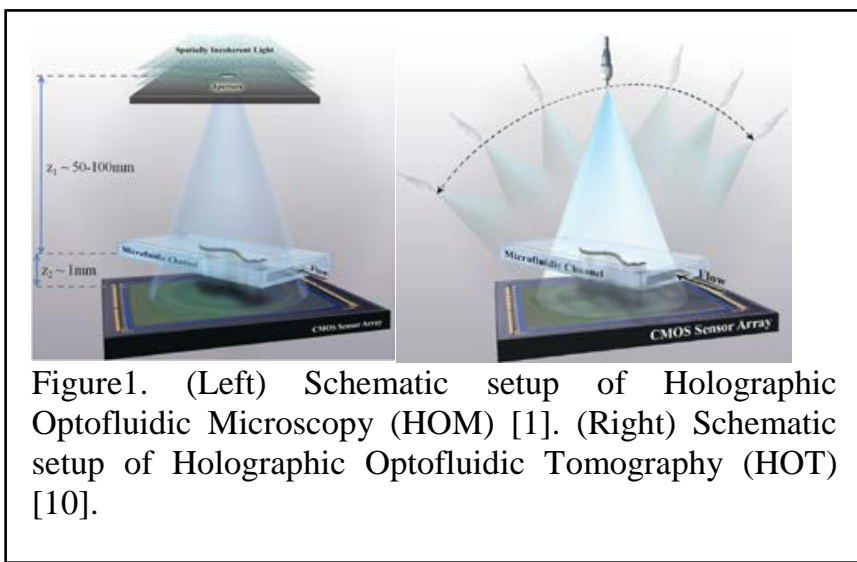

Lensless on-chip microscopy based on digital in-line holographic imaging offers a wide FOV and a large DOF within a low cost and compact device platform [1]-[10]. These features make it a promising solution for optofluidic microscopy applications [1], [6], [8]. In a holographic optofluidic microscopy (HOM) setup, the sample flows in a microfluidic channel, which is driven either by electro-kinetic force or pressure. A partially coherent light source with a large illumination aperture (e.g., $\sim 50-100 \mu \mathrm{m}$, filtered by a large pinhole or butt-coupled to an optical fiber section) is placed 50$100 \mathrm{~mm}$ ( $z_{1}$ distance) above the sample microfluidic chip, while the microfluidic chip is placed directly on top of a CMOS image sensor. As a result, the sample of interest inside the micro-channel is typically located at $\sim 1 \mathrm{~mm}\left(z_{2}\right.$ distance) away from the imager chip. The illumination light that can be from a low-cost light-emitting diode (LED) acquires spatial coherence after free-space propagation over a distance of $z_{1}$, and the hologram recorded by the image sensor is formed by the interference of the light scattered by the sample within the fluid with the unscattered light that is directly transmitted. Compared to laser sources, the use of a partially coherent light source (e.g., a simple LED) reduces speckle and multiple-interference noise, resulting in cleaner images without loss of resolution owing to on-chip imaging geometry. Moreover, the cost of the setup is reduced by using low-cost LEDs as light sources.

An important advantage of using the holographic imaging principle is the capability to do single-shot depth-resolved amplitude and phase imaging of a large sample volume. The holographic patterns can be digitally refocused to different $\mathrm{z}$ distances, with particles/samples residing at different depths respectively in focus.

When only one in-line hologram is used for reconstruction, the major limiting factor for the resolution is the pixel size, which is a disadvantage of using unit fringe magnification - a characteristic signature of an on-chip holographic microscope. However, the flow of the sample in a microfluidic 


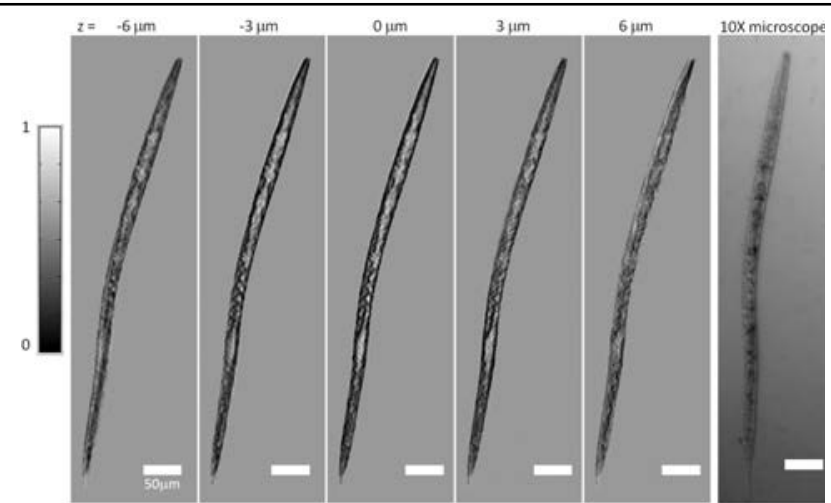

Figure 2. 3D imaging of C. elegans using HOT.[10]

channel (Fig. 1) can be leveraged to circumvent this limit imposed by the pixel size by capturing multiple holograms that are acquired at different time instances [1], [6], [8]. Due to the flow, these holograms generally have non-integer (sub-pixel) shifts with respect to each other. A high-resolution hologram that is compatible with all the sub-pixel-shifted low-resolution holograms can be calculated by using a pixel super-resolution (PSR) algorithm.

Using lens-free holographic on-chip imaging together with computed tomography principles, portable tomographic microscopes can also be built, to achieve 3D sectional imaging capability [3], [6]-[8], [10]. In this method, the sample is illuminated from multiple angular positions ranging between $\pm 50^{\circ}$, which provides different perspectives of the samples. These images are holographically reconstructed, and a computed tomogram is generated by applying a filtered back-projection algorithm. Although the multi-angle illumination increases the system complexity, it can still be implemented in a cost-effective and portable device by using multiple LEDs that are butt-coupled to optical fiber sections at different angular positions. PSR can be integrated onto the same platform by electromagnetically actuation of the optical fibers using coils and magnets [3]. This induced motion in the optical fibers results in sub-pixel shifts of the acquired holograms, and can be used to synthesize higher-resolution holographic images. With this approach, tomographic imaging of approximately 15 $\mathrm{mm}^{3}$ has been achieved, with a spatial resolution of $<1 \mu \mathrm{m} \times<1 \mu \mathrm{m} \times<3 \mu \mathrm{m}$ in $\mathrm{x}, \mathrm{y}$ and $\mathrm{z}$ direction. Tomographic imaging of an entire $C$. elegans has been demonstrated using the same technique [7].

A unique advantage of the holographic optofluidic tomography (HOT) method (Figs. 1-2) is its extended DOF that is inherent to the holographic imaging principle. Unlike high-NA objective lenses that have a very shallow DOF, a tomogram of an object can be computed at any position within the imaged 3D volume of the micro-channel. However, notice that HOT technique assumes that the sample is a weak scattering object, and that the majority of the detected photons should have experienced at most a single scattering event.

A variant of the HOM and HOT methods was used for the dynamic 3D tracking of sperm cells over large volumes [4], [9]. In this system, two LED sources of different wavelengths (red and blue) simultaneously illuminate the microfluidic chamber from different perspectives (vertical and $45^{\circ}$ tilted, respectively). This dual-wavelength illumination design enables digital separation of the two holograms of the same sperm cell, and the dual-angle design improves the cell localization accuracy in the $\mathrm{z}$ direction via triangulation. High frame rate lensless image sequences were taken to resolve the rapid motion of the sperms within a very large volume of $\sim 10 \mu \mathrm{L}$. Using this platform, rare trajectories of human sperms such as helical patterns were detected and quantified. A similar platform can be used to detect and track a wide variety of other micro-swimmers that exhibit 3D locomotion in fluids.

\section{Concluding Remarks and Perspectives}

Lensless holographic microscopy and tomography are promising techniques to complement microfluidic and lab-on-a-chip platforms as they present important advantages of e.g., high throughput, low cost, simplicity and small footprints, making them ideallysuited to be used in low-resource and field settings. Manipulation of liquid samples and rapid screening of large volumes of fluids are some of the applications that can benefit from these computational imaging techniques.

Acknowledgments - The Ozcan Research Group at UCLA gratefully acknowledges the support of the Presidential Early Career Award for Scientists and Engineers (PECASE), the Army Research Office (ARO; W911NF-13-1-0419 and W911NF-13-1-0197), the ARO Life Sciences Division, the National Science Foundation (NSF) CBET Division Biophotonics Program, the NSF Emerging Frontiers in Research and Innovation (EFRI) Award, the NSF EAGER Award, NSF INSPIRE Award, NSF Partnerships for Innovation: Building Innovation Capacity (PFI:BIC) Program, Office of Naval Research (ONR), the National Institutes of Health (NIH), the Howard Hughes Medical Institute (HHMI), Vodafone Americas Foundation, the Mary Kay Foundation, Steven \& Alexandra Cohen Foundation, and KAUST.

\section{References}

[1] W. Bishara, H. Zhu, and A. Ozcan, "Holographic opto-fluidic microscopy,” Opt. Express, vol. 18, no. 26, pp. 27499-27510, Dec. 2010. 
[2] S. O. Isikman, I. Sencan, O. Mudanyali, W. Bishara, C. Oztoprak, and A. Ozcan, "Color and monochrome lensless on-chip imaging of Caenorhabditis elegans over a wide field-of-view," Lab Chip, vol. 10, no. 9, pp. 1109-1112, May 2010.

[3] S. O. Isikman, W. Bishara, U. Sikora, O. Yaglidere, J. Yeah, and A. Ozcan, "Field-portable lensfree tomographic microscope," Lab. Chip, vol. 11, no. 13, pp. 2222-2230, 2011.

[4] T.-W. Su, L. Xue, and A. Ozcan, "High-throughput lensfree 3D tracking of human sperms reveals rare statistics of helical trajectories," Proc. Natl. Acad. Sci., vol. 109, no. 40, pp. 16018-16022, Oct. 2012.

[5] S. Seo et al., "High-Throughput Lens-Free Blood Analysis on a Chip," Anal. Chem., vol. 82, no. 11, pp. 4621-4627, Jun. 2010.

[6] S. O. Isikman et al., "Lensfree On-Chip Microscopy and Tomography for Biomedical Applications," IEEE J. Sel. Top. Quantum Electron., vol. 18, no. 3, pp. 1059-1072, May 2012.

[7] S. O. Isikman et al., "Lens-free optical tomographic microscope with a large imaging volume on a chip,” Proc. Natl. Acad. Sci., vol. 108, no. 18, pp. 7296-7301, May 2011.

[8] W. Bishara, S. O. Isikman, and A. Ozcan, "Lensfree Optofluidic Microscopy and Tomography," Ann. Biomed. Eng., vol. 40, no. 2, pp. 251-262, Feb. 2012.

[9] T.-W. Su, S. O. Isikman, W. Bishara, D. Tseng, A. Erlinger, and A. Ozcan, "Multi-angle lensless digital holography for depth resolved imaging on a chip,” Opt. Express, vol. 18, no. 9, pp. 9690-9711, Apr. 2010.

[10] S. O. Isikman, W. Bishara, H. Zhu, and A. Ozcan, “Optofluidic Tomography on a Chip,” Appl. Phys. Lett., vol. 98, no. 16, p. 161109, Apr. 2011. 


\section{Live cell imaging with optofluidics - Adam} Wax

Duke University

\section{Relevance of the topic}

Cell biologists and microscopists will often image individual live cells to establish the properties of a population of interest. However, these studies can be limited by the number of cells that can be practically examined due to the need to reposition, refocus and image each cell. As an alternative, flow cytometry can enable large throughput studies but typically requires a degree of instrument complexity. Perhaps a greater limitation is that flow cytometry makes it difficult to follow the fate of individual cells. As an alternative approach, optofluidics offer a unique opportunity to examine live cells and easily manipulate them in a high throughput scheme.

Many microfluidic platforms have been developed for examining individual live cells. The earliest implementations often included an optical scheme for interrogating a cell, usually in the form of a fluorescent reporter [1]. As the technology matured, optics were also adapted for implementing cell sorting [2]. The development of optics integrated into the microfluidic platform birthed the field of optofluidics. The first key advances in this field were to enable high resolution imaging within the microfluidic platform. These lowcost implementations offered fit for purpose alternatives to using a traditional microscope [3]. This approach combines microfluidics for sample manipulation with low cost sensors for imaging, and employ reconstruction algorithms to obtain highresolution images. Further development of these approaches for cell imaging in a compact, low cost platform has enabled applications in new settings such as resource limited locations and point of care medical diagnosis. Below, recent advances in live cell imaging using optofluidics are discussed alongside a brief look at challenges in this field and suggestions on how future efforts may approach them.

\section{Current Status and Challenges}

The true advantage of optofluidics for live cell imaging is the ability to apply a range of well controlled, calibrated forces both to manipulate and characterize cells. These forces can be applied mechanically, electrically or optically to isolate and interrogate individual cells for study. Cells under examination in these devices are then typically profiled using an optical modality ranging from bright field imaging to Raman spectroscopy to interferometry.

A recent example of this approach can be found in Lee and Liu [4] where individual cells are trapped at designated sites in a micropipette array using flow

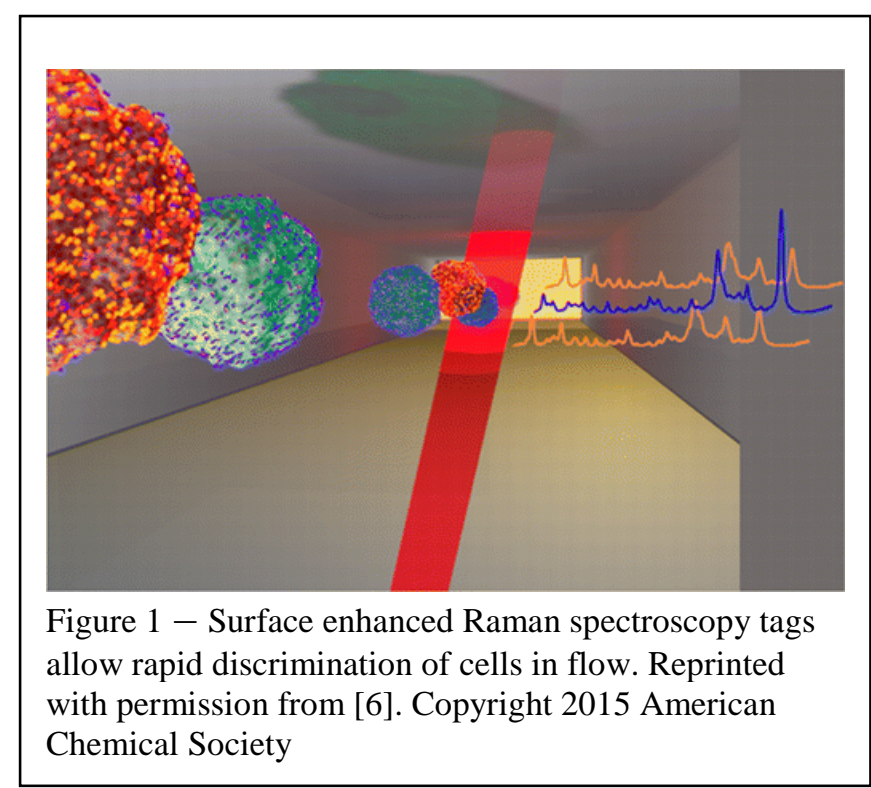

pressure. A calibrated syringe allows the pressure to be varied across the array and thus implement simultaneous micropipette aspiration assays on each trapped cell. This is a common technique in cell biology for assessing cell biomechanical properties by noting the degree to which a cell is drawn into a narrow capillary due to a given force. This technique is effective but can be quite laborious due to the need to manually trap and image each cell. In comparison, the optofluidic platform allows highly parallel studies using brightfield microscopy to assess cell deformation. In its initial demonstration, it was applied to distinguish breast cancer cells based on mechanical properties. One particular challenge of this approach is to keep the sample in focus during experiments. Here this is solved by integrating the platform with the microscope but other approaches are discussed below.

Raman spectroscopy is a powerful technique for distinguishing cells by their biochemical properties. However, in general the Raman cross section is low resulting in poor efficiency and a need for long integration times. Analysis of the Raman signal from cells inside an optofluidic platform usually must include a mechanism for trapping the cells using mechanical or electrophoresis approaches. Using laser tweezers to trap cells for Raman measurements has also been shown to be effective [5]. As an alternative approach surface-enhanced Raman spectroscopy (SERS) has been used to discriminate cells in a microfluidic platform [6]. SERS increases the strength of a Raman signal by orders of magnitude, enabling more rapid detection. In this approach a SERS biotag is created which is taken up by cells which are then introduced to the microfluidic. The flowing cells can then be rapidly profiled using the biotag for sorting.

Several efforts seek to increase the rate of cell imaging as they flow through a device. While the use of today's high pixel count sensors can allow for highly 
parallel imaging approaches, these typically are limited by the low numerical aperture optics required to have a wide field of view. Light sheet imaging approaches have shown promise for wide field and rapid throughput but are limited to detecting fluorescent tags. Novel imaging approaches, such as optical time stretch imaging allows for more rapid imaging of cells [7], achieving rates comparable to flow cytometers. In this approach the imaging information is spectrally encoded using a dispersed broadband pulse. Extensions of this approach have shown the ability to obtain phase information using an interferometry scheme but can have difficulty with maintaining cells in a focal plane. In addition, the complex scheme may not be compatible with typical applications of microfluidics as point of care devices.

\section{Advances in Science and Technology to Meet Challenges}

The continuing challenge in imaging of live cells in optofluidics is to achieve high throughput without sacrificing information or greatly increasing instrument complexity. Optofluidic devices become less efficient when there is a need to first trap each individual cell before analyzing it. Instead, developing methods in optofluidics seek to provide richer information while preserving throughput.

Holographic imaging offers a compelling way to obtain more information about cells in that it provides both phase and amplitude information on the optical field that has interacted with a cell. Unlike Raman spectroscopy or fluorescence, interferometric signals are quite robust yet the method does present some challenges. For example, cell samples in a microfluidic may present some turbidity, due to presence of other cells or fouling biomolecules. To implement holographic imaging in this situation, a clean background image is needed for subtraction from later images. A recent study pointed the way to conquering turbidity through careful analysis of the holographic signal components [8]. The approach, termed multi-look, allowed for successful imaging of cells in the face of a turbid sample in the microfluidic channel.

Another compelling aspect of holography is its ability to image the three dimensional distribution of refractive index, an indicator of the cell's mass distribution. However, this approach for refractive index cell tomography can require many camera exposures, typically from different angles, during which the cell must remain stationary. A recent advance has pointed the way to 3D holographic imaging in flow [9]. In this approach a cell flows slowly past a high speed camera which captures the angular distribution of scattered light rather than a

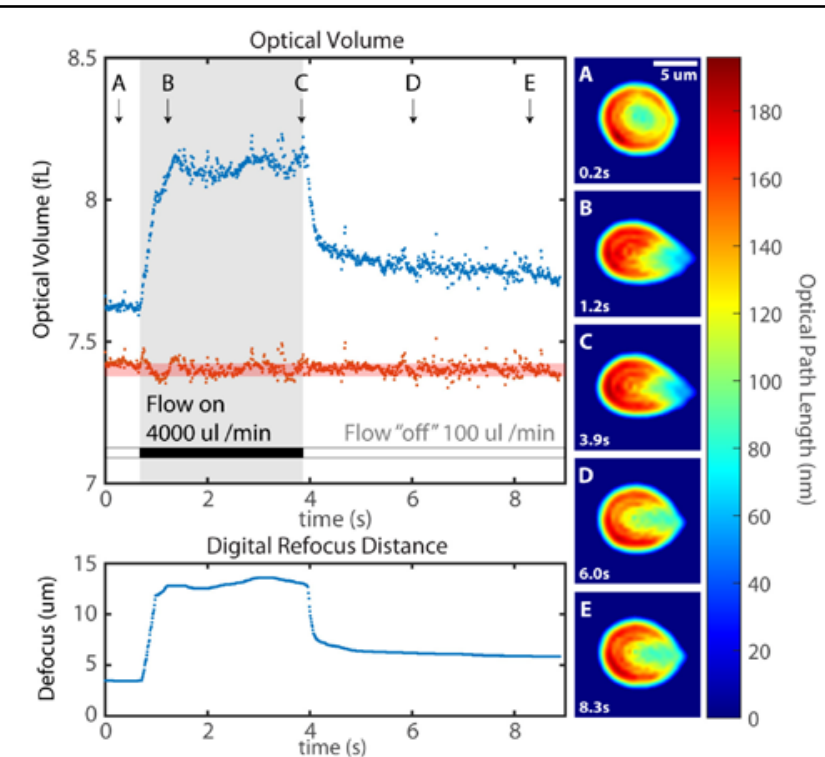

Figure 2 - Digital refocusing of a single red blood cell image subjected to high flow rates. Top-Left: Optical volume of RBC with flow on and off (rates indicated on plot). Blue, red are before, after digital refocusing. Bottom-Left: Defocus distance. Reprinted from [10] with permission.

regular image. These data are then inverse transformed to deduce the 3D distribution. This advance was significant since it first demonstrated 3D tomographic imaging of cells in flow. However, it had some limitations, including requiring many exposures and significant computation time, indicating that further development is needed for high throughput application. Further, this platform avoided the refocusing problem by confining cells to a channel just barely larger than their size, which could become easily clogged if debris were present.

As an alternative, our research group has shown that refocusing of holographic cell images can avoid the need for restrictive channel dimensions. In this approach holographic cell images are acquired and then digitally refocused by leveraging the fact that the complex optical field is obtained [10]. In this initial demonstration, it was shown that quantitative comparison of red blood cells was much more accurate after refocusing. We further employed this refocusing algorithm in two studies of red blood cells infected with $P$. falciparum, a parasite responsible for malaria. The first study used our quantitative phase spectroscopy technique [11] to analyze the hemoglobin content of individual red blood cells, revealing its consumption by the parasite [12]. A second study used machine learning to discriminate the presence of infection by using a set of cell descriptors, producing high enough sensitivity and specificity to suggest clinical utility[13]. Both of these studies employed the refocusing approach which will be essential in 
development of holographic techniques for studying cells using optofluidics.

\section{Concluding Remarks and Perspectives}

In summary, optofluidics offers unique capabilities for high throughput cell studies. Many research efforts are underway to mine the richness of the optical spectrum to characterize cells while maintaining sufficient throughput to implement viable assays. New techniques such as holographic imaging can provide new capabilities but successful application can pose new challenges as well.

Acknowledgments - Grant support from the National Science Foundation CBET 1604562.

\section{References}

[1] Wheeler, A.R., W.R. Throndset, R.J. Whelan, A.M. Leach, R.N. Zare, Y.H. Liao, K. Farrell, I.D. Manger, and A. Daridon, Microfluidic Device for Single-Cell Analysis. Analytical Chemistry, 2003. 75(14): p. 3581-3586.

[2] Wang, M.M., E. Tu, D.E. Raymond, J.M. Yang, H. Zhang, N. Hagen, B. Dees, E.M. Mercer, A.H. Forster, and I. Kariv, Microfluidic sorting of mammalian cells by optical force switching. Nature biotechnology, 2005. 23(1): p. 83-87.

[3] Cui, X., L.M. Lee, X. Heng, W. Zhong, P.W. Sternberg, D. Psaltis, and C. Yang, Lensless highresolution on-chip optofluidic microscopes for Caenorhabditis elegans and cell imaging. Proceedings of the National Academy of Sciences, 2008. 105(31): p. 10670-10675.

[4] Lee, L.M. and A.P. Liu, A microfluidic pipette array for mechanophenotyping of cancer cells and mechanical gating of mechanosensitive channels. Lab on a Chip, 2015. 15(1): p. 264-273.

[5] Lau, A.Y., L.P. Lee, and J.W. Chan, An integrated optofluidic platform for Raman-activated cell sorting. Lab on a Chip, 2008. 8(7): p. 1116-1120.

[6] Pallaoro, A., M.R. Hoonejani, G.B. Braun, C.D. Meinhart, and M. Moskovits, Rapid Identification by Surface-Enhanced Raman Spectroscopy of Cancer Cells at Low Concentrations Flowing in a Microfluidic Channel. ACS Nano, 2015. 9(4): p. 4328-4336.

[7] Yang, T., P. Paiè, G. Nava, F. Bragheri, R.M. Vazquez, P. Minzioni, M. Veglione, M. Di Tano, C. Mondello, and R. Osellame, An integrated optofluidic device for single-cell sorting driven by mechanical properties. Lab on a Chip, 2015. 15(5): p. 1262-1266.

[8] Bianco, V., F. Merola, L. Miccio, P. Memmolo, O. Gennari, M. Paturzo, P.A. Netti, and P. Ferraro, Imaging adherent cells in the microfluidic channel hidden by flowing RBCs as occluding objects by a holographic method. Lab Chip, 2014. 14(14): p. 2499-504.

[9] Sung, Y., N. Lue, B. Hamza, J. Martel, D. Irimia, R.R. Dasari, W. Choi, Z. Yaqoob, and P. So, ThreeDimensional Holographic Refractive-Index
Measurement of Continuously Flowing Cells in a Microfluidic Channel. Physical Review Applied, 2014. 1(1): p. 014002.

[10] Rinehart, M.T., H.S. Park, and A. Wax, Influence of defocus on quantitative analysis of microscopic objects and individual cells with digital holography. Biomedical optics express, 2015. 6(6): p. 2067-2075.

[11] Rinehart, M., Y. Zhu, and A. Wax, Quantitative phase spectroscopy. Biomedical optics express, 2012. 3(5): p. 958-965.

[12] Rinehart, M.T., H.S. Park, K.A. Walzer, J.-T.A. Chi, and A. Wax, Hemoglobin consumption by $P$. falciparum in individual erythrocytes imaged via quantitative phase spectroscopy. Scientific reports, 2016. 6.

[13] Park, H.S., M.T. Rinehart, K.A. Walzer, J.-T.A. Chi, and A. Wax, Automated Detection of $P$. falciparum Using Machine Learning Algorithms with Quantitative Phase Images of Unstained Cells. PloS one, 2016. 11(9): p. e0163045. 


\section{Optofluidic microlenses: from tunable} focal length to aberration control - Frieder Mugele

University of Twente

\section{Relevance of the topic}

Generating optical images by refractive lenses is still the most important practical use of optical systems. In principle only one single lens is needed to form an optical image and two to generate a zoom system. In practice, however, optical systems require a lot more than two lenses in order to guarantee a decent ideally: diffraction limited - imaging quality. Combinations of several lenses are typically used to compensate imaging faults that arise from the various aberrations of each individual lens. If zoom or focus settings are to be changed, many of these lenses have to be displaced with respect to each other in a welldefined manner. This typically requires fairly precise and bulky mechanical displacement stages that are often not compatible with, e.g., the spatial constraints in high tech devices such as mobile phones or endoscopes.

Optofluidic lenses provide a way out of this dilemma. Instead of translating lenses they adapt their imaging properties by adapting the shape of the lenses. This can be achieved by a variety of external control parameters including pressure variations, mechanical stresses, thermal expansion, and electrowetting [3]. While these approaches provide a fair degree of tunability of the focal length, most of them retain a spherical shape of the lens, as dictated by the mechanical equilibrium of free liquid surfaces in the absence of external forces. As a consequence, optical systems built of simple optofluidics lenses often display mediocre imaging quality due to uncompensated imaging faults such as spherical and other optical aberrations. High quality adaptive imaging systems require new strategies for aberration correction in liquid microlenses while preserving the compactness of the design, i.e. without introducing mechanical stages to displace the lenses.

\section{Current Status and Challenges}

Standard optofluidic lens designs come in two basic variants as illustrated in Fig. 1a and b. In the first type, the edge of the lens, i.e. the three phase contact line between the solid, the lens fluid (LF) and the ambient fluid (AF) is pinned along the edge of a physical aperture, Fig. 1a. In this case, the curvature $\boldsymbol{\kappa}$ of the lens is determined either by the pressure difference $\boldsymbol{\Delta} \boldsymbol{p}_{\boldsymbol{L}}=\mathbf{2} \boldsymbol{\gamma} \boldsymbol{\kappa}$ between the two fluids or by the fixed volume of the lens fluid in combination with geometric constraints. The interfacial tension $\boldsymbol{\gamma}$ between the two fluids guarantees the spherical cap shape of the interface. (The densities of lens and ambient fluid are

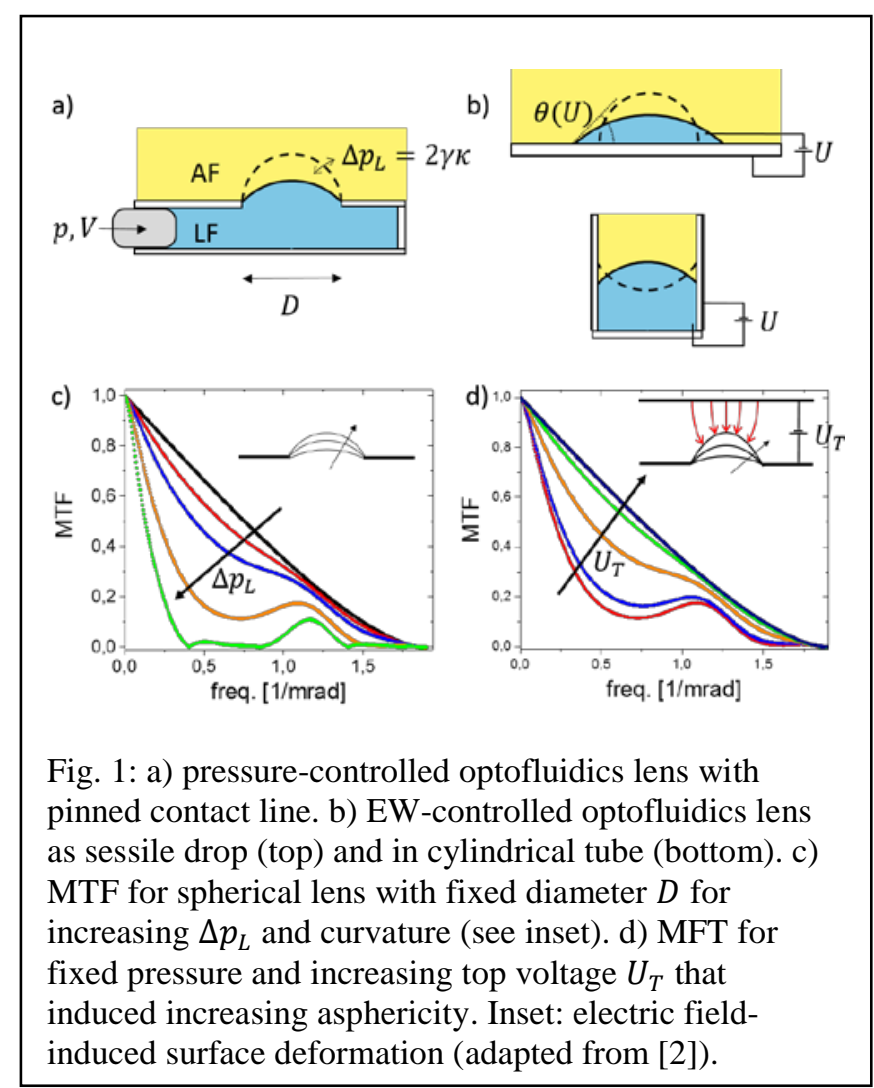

typically matched in order to avoid gravity-induced distortions.) Pressure and/or volume can be changed by a variety of different actuation schemes including piezo-electric transducers, thermal expansion, and electrowetting, see [3] for a recent review. In some approaches, the interface between the two fluids is covered by an elastomeric membrane, e.g. of PDMS (polydimethylsiloxane) with a thickness of a few tens of micrometers. For not too large deformations, the resulting shape of the interface in these 'liquid-filled' lenses is also approximately spherical cap-shaped with the elastic tension of the membrane taking over the role of $\gamma$.

In the second variant of optofluidics lenses, the three phase contact line is free to move along the solid surface, but the contact angle $\boldsymbol{\theta}$ is changed, Fig. $1 \mathrm{~b}$. Variations of $\boldsymbol{\theta}$ in combination with the fixed radius and/or volume of the lens fluid determine the curvature of the lens. Reversible, fast, and robust tuning of the contact angle over a wide range is best achieved using electrowetting (EW; see [5] for a review). EW requires of a conductive lens fluid and a dielectric ambient fluid. It allows to vary $\boldsymbol{\theta}(\boldsymbol{U})$ by more than $90^{\circ}$ within milliseconds with excellent reproducibility over millions of actuation cycles.

Yet, both approaches lead to spherical lenses for cylindrically symmetric geometries. As a consequence, such lenses always suffer from optical (in particular: spherical) aberrations that compromise the resulting image quality, as evidenced in plots of the modulus of the optical transfer function (MTF) for increasing 
curvature, Fig. 1c, and in the resulting images, Fig. 2a. Such deteriorations are particularly dramatic for lenses with high numerical aperture or small $f$-number, as they are frequently needed in miniaturized devices.

In the case of liquid-filled lenses, combinations of two differently curved surfaces in biconvex lenses were demonstrated to reduce both spherical $[6,7]$ and chromatic aberration. The different curvatures are achieved by using two membranes with a different stiffness resulting in different curvatures for the same applied pressure, or more recently with elastomeric membranes with a carefully designed radial thickness profile [8]. While preserving the elegance of a single actuator, the applied pressure, this fixed design of the membranes, and their non-linear elastic and hysteretic response, reduce to some extent the flexibility of this approach.

\section{Advances in Science and Technology to Meet Challenges}

To overcome these limitations, alternative actuation schemes that enable not only a variation of the overall average curvature of the lens, but also controlled deviations from the spherical shape are needed. A few recent studies suggest to address this problem using electrowetting or a related electrical actuation in combination with suitably patterned electrodes. One of the first applications of this type was not intended for imaging optics but for (wide angle) beam steering. Smith et al.[9] used square or rectangular cuvettes with EW-functionalized sidewalls that could be addressed individually. In this manner, they managed to generate flat liquid-liquid interfaces with a widely tunable tilt angle resulting in a $\mathbf{\pm 1 5 ^ { \circ }}$ beam steering capability.

Kopp and Zappe [4] recently presented a design in which they segmented the electrode along the inner side of a cylinder. In this case, the liquid-liquid interface assumes in general complex shape that is compatible with the local contact angles on electrode and the requirement of a constant mean curvature in mechanical equilibrium. Using eight individually addressable electrode segments they managed to generate lenses with tunable focal length $(7-12 \mathrm{~mm})$ and tunable astigmatism in $0, \mathbf{\pm 4 5 ^ { \circ }}$, and $90^{\circ}$ directions with amplitudes up to $3 \mu \mathrm{m}$ (Zernike coefficient) while keeping the amplitude of all other aberrations below 100nm, Fig. 2b.

Mishra et al. [1] presented a different approach, in which they used another electrode placed in the ambient dielectric above the liquid-liquid interface, see inset Fig. 1d. Application of a high voltage to that electrode generates a position-dependent electric field that pulls on the liquid-liquid interface with a Maxwell stress $\boldsymbol{\Pi}(\boldsymbol{r})=\boldsymbol{\epsilon}_{\mathbf{0}} \boldsymbol{\epsilon} \boldsymbol{E}^{\mathbf{2}}(\boldsymbol{r}) / \mathbf{2}$. The equilibrium shape of the liquid is set by balance between this position-
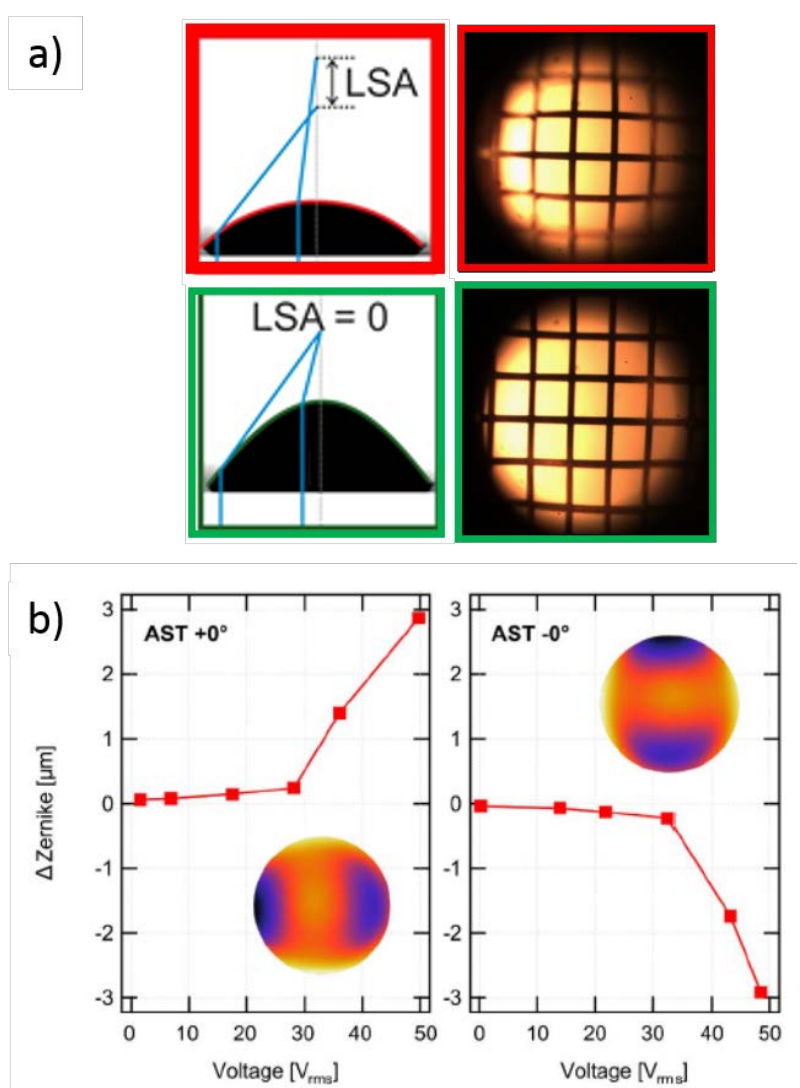

Figure 2: a) top: side view image (left) and image of a test grid (right) recorded with a spherical liquid lens with finite longitudinal spherical aberration (LSA); bottom: same as in a) for an aspherical lens for negligible LSA deformed by optimized Maxwell stress. (adapted from [1]) b) Zernike coefficient for astigmatism in vertical and horizontal direction obtained by EW with segmented electrodes in azimuthal direction. (reproduced from [4])

dependent electrical stress and the resulting positiondependent local Laplace pressure and curvature of the interface. For an unstructured electrode on the top, the local curvature of the interface decreases monotonically from the optical axis towards the edge of the lens, similar to a perfect aspherical lens. Indeed, it turns out that the longitudinal spherical aberration can be completely eliminated, resulting in diffractionlimited MTF curves, Fig. 1d, as well as optical images (Fig. 2b).

Extensions of the method using segmented top electrodes, a homogeneous one, allow to break the cylindrical symmetry and the generate lenses with tunable astigmatism [10], coma, and various other aberrations.

\section{Concluding Remarks and Perspectives}

EW and the related Maxwell stress-induced tuning of liquid-liquid interfaces provide very fast and flexible tools to generate tunable lens profiles with variable local curvature. The design of optofluidics devices that allow for correcting tilt, coma, astigmatism, and 
spherical aberration simultaneously with an all electrical actuation seems within reach, possibly best by combining the currently existing approaches discussed above. The extent to which different aberrations can be addressed completely independently needs to be explored. Improved geometric designs aided by numerical simulation and improved materials (e.g. low interfacial tension fluids) will help to improve the flexibility. Ideally, it is conceivable to achieve almost arbitrary polarization-independent wavefront shaping on top of a lensing effect by using arrays of large numbers of individually addressable electrodes.

Acknowledgments - We thank the Dutch Science Foundation NWO supporting our optofluidics activities within their VICI program under grant \#11380.

\section{References (separate from the two page limit)}

[1] Mishra, K, Murade, C, Carreel, B, Roghair, I, Oh, JM, Manukyan, G, van den Ende, D, and Mugele, F, 2014 Optofluidic lens with tunable focal length and asphericity. Scientific Reports. 4:6378.

[2] Mishra, K and Mugele, F, 2016 Numerical analysis of electrically tunable aspherical optofluidic lenses. Optics Express. 24:14672-14681.

[3] Mishra, K, van den Ende, D, and Mugele, F, 2016 Recent Developments in Optofluidic Lens Technology. Micromachines. 7:102.

[4] Kopp, D and Zappe, H, 2016 Tubular astigmatismtunable fluidic lens. Optics Letters. 41:2735-2738.

[5] Mugele, F and Baret, JC, 2005 Electrowetting: From basics to applications. Journal of Physics-Condensed Matter. 17:R705-R774.

[6] Fuh, YK, Lin, MX, and Lee, S, 2012 Characterizing aberration of a pressure-actuated tunable biconvex microlens with a simple spherically-corrected design. Optics and Lasers in Engineering. 50:1677-1682.

[7] Yu, HB, Zhou, GY, Leung, HM, and Chau, FS, 2010 Tunable liquid-filled lens integrated with aspherical surface for spherical aberration compensation. Optics Express. 18:9945-9954.

[8] Zhao, PP, Ataman, C, and Zappe, H, 2016 Gravityimmune liquid-filled tunable lens with reduced spherical aberration. Applied Optics. 55:7816-7823.

[9] Smith, NR, Abeysinghe, DC, Haus, JW, and Heikenfeld, J, 2006 Agile wide-angle beam steering with electrowetting microprisms. Optics Express. 14:65576563.

[10] Lima, NC, Cavalli, A, Mishra, K, and Mugele, F, 2016 Numerical simulation of astigmatic liquid lenses tuned by a stripe electrode. Optics Express. 24:42104220. 


\section{Integration of reconfigurable photonics and microfluidics - Holger Schmidt University of California, Santa Cruz}

\section{Relevance of the topic}

Ever since the emergence of optofluidics in the early 2000s, reconfigurability has been one of the field's defining characteristics, afforded by the potential to replace and modify non-solid media to (rapidly) change the optical properties of a photonic device $[1,2]$. At the time, reconfigurable devices were inspired by possible applications in optical communications. Thus, on-chip laser sources that could be tuned via swapping the active (liquid) gain medium or mechanically deforming the cavity were a natural early demonstration of the power of this approach [3]. Subsequently, reconfigurability was demonstrated in a number of other photonic elements, including switches [4], lenses [2], microring resonators [2], and spectral filters [2].

\section{Current Status and Challenges}

Reconfigurability remains one of the distinctive characteristics and a major thrust area for optofluidics. However, over the years, the main interest in terms of real-world applications has shifted towards chemical and biological analysis, in particular disease diagnostics. Here, some of the opportunities lie in integration of photonic functions with microfluidic sample handling and the development of compact, powerful point-of-care devices. For example, recently single virus particles have been imaged on a smartphone-based platform [5]. At the same time, hybrid integration of silicon-based optofluidic chips with glass or PDMS-based microfluidic chips has emerged as a powerful approach to create a single, optofluidic system that is optimized for both sample preparation and analysis [6,7]. Using a combination of microvalve-controlled microfluidics and liquid-core waveguide-based fluorescence sensing as shown in Fig. 1a(i), amplification-free detection of single Ebola nucleic acids was demonstrated with high specificity over the entire clinically relevant viral load range [8]. Moreover, multimode interference (MMI) waveguides were integrated on the same silicon chip platform to add multiplexing capabilities by creating colordependent spot patterns in a liquid channel as illustrated in Fig. 1a(ii) [9]. This approach was successfully used to distinguish three influenza subtypes on the single virus particle level [9].

These recent demonstrations are indicative of the power of waveguide-based optofluidic devices. It is, therefore, a natural question whether the wellestablished fluidic reconfigurability [1-4] can further

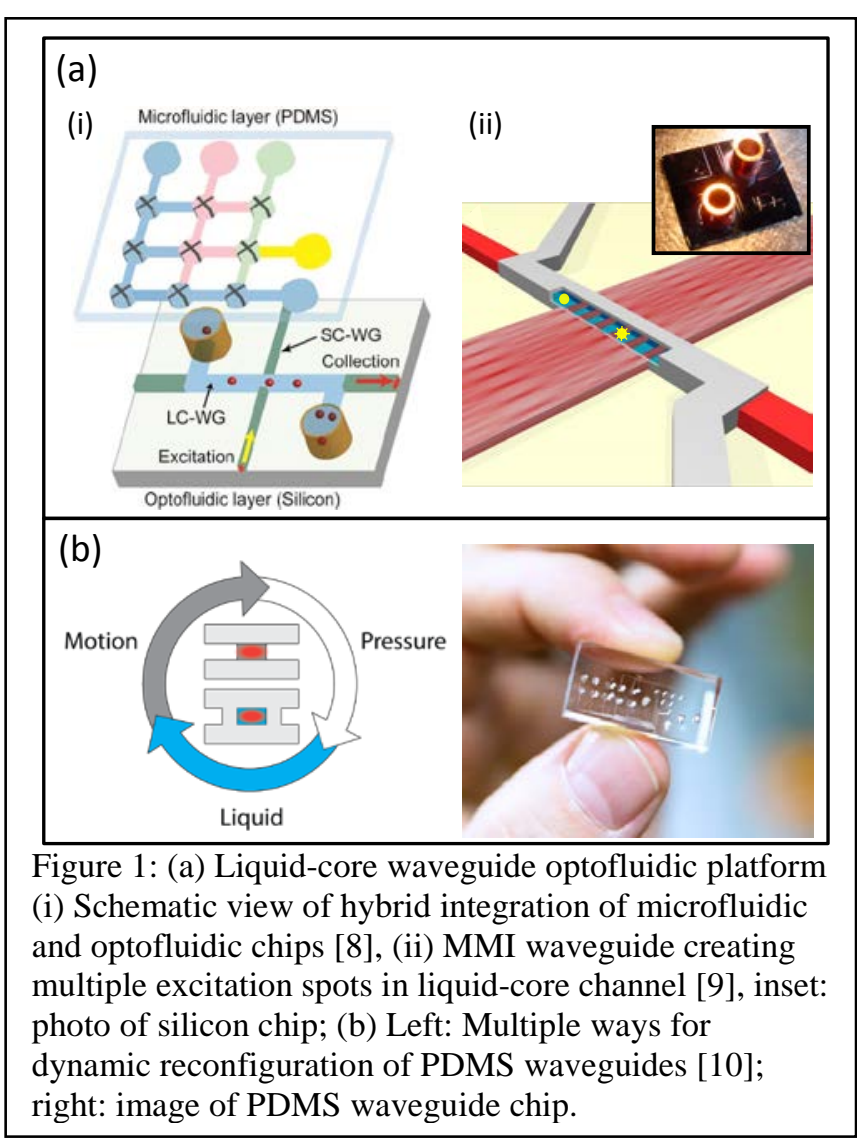

enhance emerging optofluidic platforms for advanced biochemical analysis.

\section{Advances in Science and Technology to Meet Challenges}

Devices made from flexible materials such as PDMS offer the highest potential for dynamic reconfiguration as their optical properties can be modified in at least three ways - mechanical pressure, altering the fluid in a channel, and by physical movement. These options are visualized in Fig. 1b (left). Recently, the first PDMS-based optofluidic platform that features all these options for dynamic reconfiguration was realized using a combination of fluid-filled microchannels (liquid-core waveguides) and solid-core PDMS waveguides in which the core and cladding are defined by PDMS layers with different precursor mixing ratios and, thus, different refractive indices (Fig. 1b) [10]. When properly fabricated, this allows for guiding light through micron-scale solid-core waveguides and interacting with liquid channels, much like established silicon-based devices [2,8,9]. A photograph of a completed device that features both a microvalvebased sample preparation region and an optical analysis region on the same chip is shown in Fig. 1b (right).

Fig. 2 illustrates different aspects of this powerful, new platform, highlighting the different reconfigurability options [10]. Fig. 2a summarizes the implementation of an MMI waveguide whose properties can be 


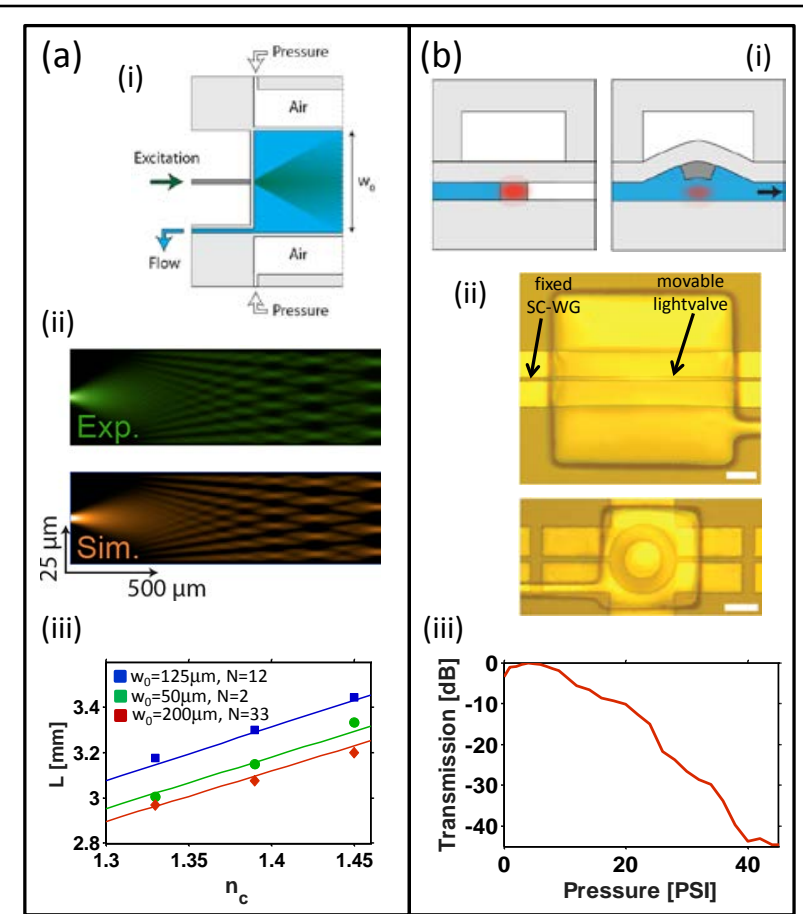

Figure 2: (a) Tunable MMI waveguide. (i) Schematic top-down view, (ii) image of light pattern in MMI section (top) in excellent agreement with simulations (bottom), (iii) tuning of spot pattern with core fluid index for different MMI widths [10]; (b) Actuatable lightvalves. (i) schematic view of cross-section of liftinggate valve with central high index layer for waveguiding, (ii) top-down images of straight waveguide on-off switch (top) and ring-shaped lightvalve for particle trapping and analysis (bottom), (iii) pressure-dependent light transmission across straight lightvalve switch from (ii) with high on-off ratio.

controlled using the core fluid or mechanical pressure. Fig. 2a(i) shows a schematic top down view where light from a single-mode solid-core PDMS waveguide section (dark gray) enters a wide channel that can be filled with different liquids and acts as the MMI waveguide. When filled with ethylene glycol, an indexguiding MMI is formed. Fluorescent dye in the solution reveals the multimode interference pattern (Fig. 2b(ii, top)) in near-perfect match with the simulated behavior of this structure (Fig. 2b(ii, bottom)). This spot pattern can be dynamically altered, either by changing the core liquid or by applying pressure in the air cavities surrounding the MMI, thus changing its width. Fig. 2a(iii) shows how the location of different spot numbers along the MMI waveguide can be tuned with the index of the liquid in the MMI section.

A novel type of reconfiguration was demonstrated in this platform by re-envisioning the design and function of fluidic lifting-gate microvalves previously used for carrying out distribution and preparation of biological sample materials [8]. Fig. 2b(i) shows a schematic cross section of such a valve where a thin PDMS membrane sits on a substrate, thus blocking fluid flow (left). However, if the segment that seals the channel (dark gray) is made of high-index PDMS, the valve also acts as an optical waveguide, symbolized by the red confined mode in the figure. The structure now acts as a "lightvalve" because lifting the central membrane up by applying negative pressure in the pneumatic top layer results in the light passing through fluid that now fills the space (Fig. 2b(i), right). This creates a new type of optical waveguide that can be individually controlled and dropped into fluidic channels at will. Fig. 2b(ii) shows two examples for the vast number of possibilities that this principle creates. The top image shows a photograph of a straight lightvalve segment that connects two fixed, solid-core waveguide sections. When resting on the substrate, light is passed with negligible loss, but upon pneumatic actuation (lifting up or pushing down), the optical path across the lighvalve becomes extremely lossy. Fig. 2b(iii) shows how the transmission can be controlled by the (pushdown) actuation pressure, and a tunable light switch with on-off ratios of up to $45 \mathrm{~dB}$ is created. This switch can be operated without degradation over 100,000 times.

On the bottom of Fig. 2b(ii), another version of the lightvalve is shown. Here, the movable segment is shaped as a ring with short waveguide stubs that interface with fixed solid-core waveguide sections on either end. This annulus was used as an actuatable trap for particles that flow through the fluidic channel when the ring is lifted. In this way, trapping and waveguidebased optical analysis of a controlled number of fluorescent beads and E.coli bacteria was demonstrated [10]. This shows that the lightvalve concept has tremendous flexibility both in geometrical design and functionality vis-a-vis transport and manipulation of both fluids and light.

\section{Concluding Remarks and Perspectives}

Dynamic reconfiguration can significantly expand the functionality and performance of waveguide-based optofluidic devices. This was illustrated with a PDMSbased platform that combines liquid-core and solidcore waveguides as well as fluidic sample handling in a single device. Light-guiding microvalves (lightvalves) that can be physically moved to alter fluid and light propagation further add to the optofluidic toolbox. It can be expected that additional novel functionalities will be demonstrated on this platform as well as using other optofluidic waveguide types such as slot waveguides, photonic crystal waveguides, or laserwritten waveguides.

Acknowledgments - This work was supported by the NIH under grants 4R33AI100229 and 1R01AI11698901 as well as the W.M. Keck Center for Nanoscale Optofluidics at UC Santa Cruz. 


\section{References}

[1] Monat C, Domachuk P, and Eggleton BJ 2007 Integrated optofluidics: A new river of light. Nat. Photonics 1, 106.

[2] Schmidt H., Hawkins AR 2011 The photonic integration of non-solid media using optofluidics. Nature Photonics 5, 598-604.

[3] Li Z, Zhang Z, Scherer A, Psaltis D 2006 Mechanically tunable optofluidic distributed feedback dye laser. Opt Express 14, 10494-9.

[4] Campbell K, Groisman A, Levy U, Pang L, Mookherjea, S,Psaltis D, and Fainman, Y 2004 A microfluidic 2x2 optical switch. Appl. Phys. Lett. 85, 6119-6121.

[5] Wei Q, Qi H, Luo W, Tseng D, Ki SJ, Wan Z, Göröcs Z, Bentolila LA, Wu TT, Sun R, and Ozcan A 2013 Fluorescent Imaging of Single Nanoparticles and Viruses on a Smart Phone ACS Nano 7, 9147-55.

[6] Parks JW, Cai H, Zempoaltecatl L, Yuzvinsky TD, Leake K, Hawkins AR 2013 Hybrid optofluidic integration. Lab Chip 13, 4118-23.

[7] Testa G, Persichetti G, Sarro PM, Bernini R 2014 A hybrid silicon-PDMS optofluidic platform for sensing applications. Biomed Opt Express 5, 417-26.

[8] Cai H, Parks JW, Wall TA, Stott MA, Stambaugh A, Alfson K, Carrion R, Patterson JL, Hawkins AR, and Schmidt H 2015 Optofluidic analysis system for amplification-free, direct detection of Ebola infection. Scientific Reports 5, 14494.

[9] Ozcelik D, Parks JW, Wall TA, Stott MA, Cai H, Parks JW, Hawkins AR, and Schmidt H 2015 Optofluidic wavelength division multiplexing for single-virus detection. Proc. Nat. Acad. Sci. 112, 12933-12937.

[10] Parks JW, and Schmidt H 2016 Flexible optofluidic waveguide platform with multi-dimensional reconfigurability. Sci. Rep. 6, 33008. 


\section{Optofluidic waveguides and resonators}

\section{- Genni Testa, Romeo Bernini CNR-IREA}

\section{Relevance of the topic}

Optofluidic waveguides offer the possibility of guiding light through a fluid that could be a liquid, a gas or a plasma. This peculiar property could be exploited in all applications in which strong light-matter interaction is required. In recent years, promising demonstration of the potentiality of optofluidic waveguides have been reported in sensing [1], non-linear photonics, atomic spectroscopy and quantum interference [2].

Daniel Colladon gave the first demonstration of an optofluidic waveguide in 1842, however, only in recent years a great effort has been devoted to the development of reliable and high performance optofluidic waveguides and devices [3]. The main issue related to optofluidic waveguides is the difficulty of fulfilling the total internal reflection (TIR) condition when low refractive index fluids are used as core material. However, optical confinement in a waveguide can be also based upon interference phenomena along the transverse direction [3]. The first Bragg fibre utilizing a 1D photonic crystal was proposed by Yeh et al. in 1978. Russell successively extended this concept in a 2D geometry. Hollow core photonic crystal (HCPC) fibers have been successfully applied. However, HCPC fibers require complex fabrication procedures and could not be easily integrated with other components. In order to overcome these problems, many integrated architectures have been proposed to develop integrated waveguides that can be considered for planar optofluidics, like slot waveguides (SWs), Bragg (BWs) or Antiresonant Reflection Optical Waveguides (ARROWs) [3] (Fig.1). The advantage of these approaches relies on the possibility to use them as building block of more complex devices. In particular, the opportunity to make optofluidic resonators has attracted a lot of attention. These devices enable a strong increase of the light-matter interaction, which is no more limited by the physical length but is related to the number of revolutions of light in the resonator, characterised by the quality factor $\mathrm{Q}$.

\section{Current Status and Challenges}

Integrated optofluidic waveguides like SWs, BWs and ARROWs offer very attractive potential of low loss, single mode operation, which is highly desirable to build photonic circuits and systems. Moreover, the planar nature of these architectures lends itself to further integration of additional functionalities, like microfluidics, in a similar manner as integrated microelectronics, leading to self-contained

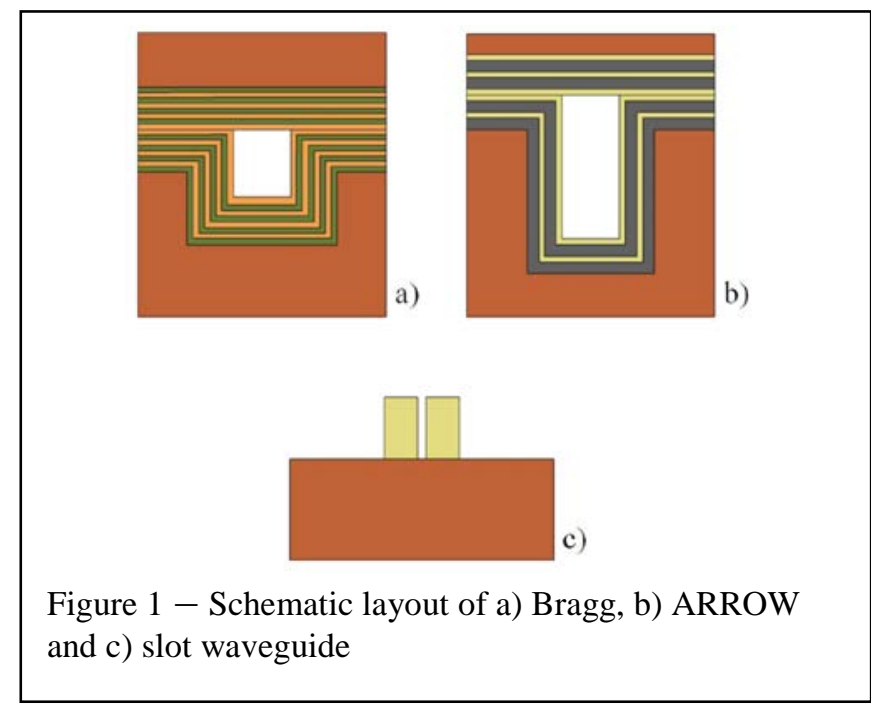

microsystems. SWs confine a substantial fraction of the optical power into nanometer void thanks to TIR effect. Such a tight confinement in subwavelength interaction area makes these waveguides very powerful for non-linear photonics and sensing applications [3]. Although SWs are extremely promising in detecting ultra-low concentrations, the fluidic transport scales unfavourably in nanofluidic channels, making the integration of fluidic system for sample manipulation still a challenging task. The implementation of integrated waveguides with hollow core is also possible by exploiting interference effects. BWs and ARROWs have been fabricated by creating multilayered dielectric claddings that act as a high performance reflector. This confinement mechanism causes propagation losses due to a not ideal boundary reflector, and thus requires an accurate design strategy. Air guiding BWs with core sizes of about $60 \times 2 \mu \mathrm{m}^{2}$ and propagation losses of few $\mathrm{dB} / \mathrm{cm}$ have been demonstrated and bent section investigated. However, the implementation of Bragg waveguides into complex circuit layouts for sensing purposes has not been fully demonstrated. Single mode, linearly polarized, liquid core ARROW with mode area of $4.5 \mu \mathrm{m}^{2}$ and low attenuations of about $1 \mathrm{~dB} / \mathrm{cm}$ have been demonstrated by an optimized design of the waveguide structure. Nonetheless, further improvements are attainable for both BWs and ARROWs that could open-up new application scenarios, especially in the field of sensing. An important concern in the waveguide performances is to reduce the core size in order to further shrink the mode field volume. Small mode volume can significantly enhance the light-matter interaction and increase the sensitivity by reducing the background optical noise. However, small core size poses tremendous design and fabrication challenges since it typically leads to a rapid increase of the propagation and bend losses.

Until now, integrated optofluidic waveguides have been mostly designed to operate in the visible/near-IR 
spectral range with liquid core $(\mathrm{n}>1.33)$. Nonetheless, they can be designed to operate with a gaseous core $(n \approx 1)$ and in a larger spectral window from the visible to the far-IR, providing that the cladding materials and the waveguide geometry are properly selected. In particular, the mid-infrared (mid-IR) $(2.5-25 \mu \mathrm{m})$ is very interesting as it supports strong molecular absorption fingerprints, thus offering an interesting spectral window for gas/vapour spectroscopy as the hollow core can be used as micro chamber, enabling a strong sensitivity enhancement. Promising results with slot waveguides operating in the mid-IR for gas and liquid sensing have been proposed [4]. In the case of ARROWs, when the core is filled with gases, the design optimization as far as the fabrication turns into a nontrivial task, which is still being investigated.

A promising strategy to further improve the light-matter interaction is to increase the duration of the interaction by placing the sample instead of target inside an optical cavity performing a high quality factor Q. Thus far, various types of optical resonators have been demonstrated in optofluidic format. Optofluidic resonators in the form of planar ring have been obtained using SWs [5] and ARROWs [6] (Fig. 2), but also in the form of Fabry-Pérot (FP) [7]. Each type of implementation has its own suitable application. Optofluidic resonators based on slot and hollow core waveguides, despite having moderate Qfactors (up to $10^{4}$ ), open up interesting perspectives for planar integration. Nowadays the increase of the Qfactor still remains a challenging task due to the leaky nature of the interference based optofluidic waveguides and the problem associated with fabrication roughness into the nanometer-void of the SWs. The availability of high Q-factor resonators could represent a paradigm shift from passive to active sensors based on lasing action. Recent results have demonstrated that optofluidic lasers could result in a promising way towards ultrasensitive detection [7].

\section{Advances in Science and Technology to Meet Challenges}

The need to reduce the waveguides losses requires a twofold approach. First, the development and the optimization of new materials and refined fabrication processes. The proper selection of the cladding materials and deposition processes has proven to effectively reduce waveguides losses. Second, the development of novel waveguide configurations able to overcome the limitations of existing designs. Atomic Layer Deposition (ALD) has been demonstrated to be a powerful tool to reduce loss in ARROWs [8], but also to realize novel slot waveguides [9]. New waveguide layouts are needed to extend the working range of optofluidic waveguides from vis-IR to mid-IR region that can offer new integrated solutions to overcome the

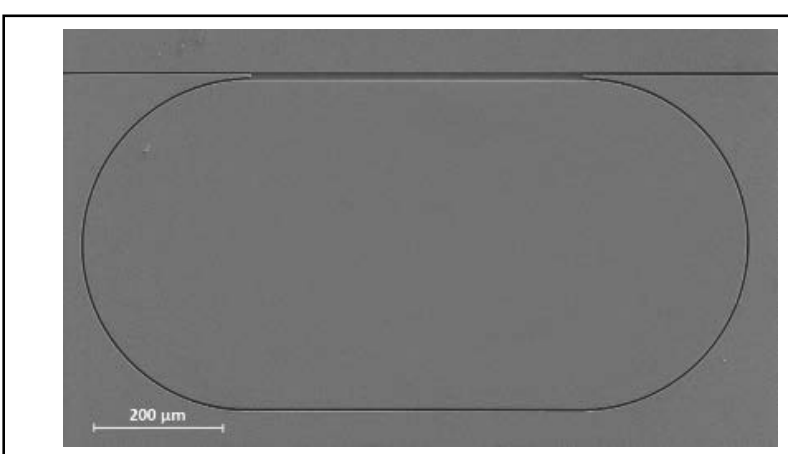

Figure 2 - Scanning electron microscope picture of an optofluidic ring resonator based on ARROWs.

limited material choice of the integrated technologies in the mid-IR. As an example, suspended silicon slot waveguides have been proposed [4].

Concerning optofluidic resonators, there are two main objectives to address in order to improve their performances and effectiveness. The first is to improve the quality factor of the resonator by optimizing the different optical elements (waveguide, bend regions, coupler) as also discussed above. The second is to provide a fine manipulation of the sample fluid by integrating suitable microfluidic systems. From this point of view, polymer materials offer several advantages for microfluidics like reduced cost and simplicity of fabrication. For this reason, hybrid silicon/polymer approaches appear as an optimal design strategy [6],[10], requiring, however, still considerable fabrication efforts to fully merge silicon photonics and microfluidics.

\section{Concluding Remarks and Perspectives}

In perspective, optofluidic waveguides have the potentiality to play the same role as solid-core waveguides do for conventional integrated photonic circuits. Promising performances have been demonstrated through the implementation of innovative waveguide layouts and high-yield fabrication processes. Ongoing progress is also focused to extend the range of applicability to the mid-IR range in order to address new applications including gas sensing.

The tasks of improving their performance and the integration on a chip are nowadays the main challenges that could be addressed in the near future by further development of state-of-the art modelling and manufacturing. By taking advantage of recent innovative polymeric manufacturing techniques, hybrid silicon/polymer approaches will pave the way to the integration of additional functionalities on chip. Moreover, polymer technologies could open the prospect of high performance and low cost all-plastic optofluidic microsystems, also suited to disposable applications. 
Acknowledgments - The authors acknowledge the financial support from the Italian Minister of University and Research (MIUR) through the Futuro in Ricerca (FIR) Program under Grant RBFR122KL1.

\section{References}

[1] Testa G, Persichetti G and Bernini R 2015 Optofluidic approaches for enhanced microsensor performances Sensors 15 465-484.

[2] Schmidt H and Hawkins A R 2010 Atomic spectroscopy and quantum optics in hollow-core waveguide Laser \& Photon Rev 4 720-737.

[3] Schmidt H and Hawkins AR 2008 Optofluidic waveguides: I. Concepts and implementations Microfluid Nanofluid 4 3-16.

[4] Lin $P$ T, Kwok S W, Lin H-Y G, Singh V, Kimerling L C, Whitesides G M and Agarwal A 2014 Mid-Infrared Spectrometer Using OptoNanofluidic Slot-Waveguide for Label-Free OnChip Chemical Sensing Nano Letters 14, 231-238

[5] Barrios C A, Bañuls M J, González-Pedro V, Gylfason K B, Sánchez B, Griol A, Maquieira A, Sohlström H, Holgado M and Casquel R 2008 Label-free optical biosensing with slot-waveguides Opt Lett 33 708-710.

[6] Testa G, Collini C, Lorenzelli L and Bernini R 2016 Planar Silicon-Polydimethylsiloxane optofluidic ring resonator sensors IEEE Photon Tech Lett $\mathbf{2 8}$ 155-158.

[7] Fan X and Yun S-H 2014 The potential of optofluidic biolasers Nature Methods 11 141-147.

[8] Testa G, Huang Y, Zeni L, Sarro P M and Bernini R 2010 Liquid Core ARROW Waveguides by Atomic Layer Deposition IEEE Photon Tech Lett 22 616618.

[9] Häyrinen M, Roussey M, Säynätjoki A, Kuittinen M and Honkanen S 2015 Titanium dioxide slot waveguides for visible wavelength Appl Opt $\mathbf{5 4}$ 2653-2657.

[10] Testa G, Persichetti G, Sarro P M and Bernini R 2014 A hybrid silicon-PDMS optofluidic platform for sensing applications Biomed Opt Express 5 417426. 


\section{High-content physical phenotyping of biological objects with microfluidic dual- beam laser traps - Jochen Guck \\ Technische Universität Dresden}

\section{Relevance of the topic}

Dual-beam laser traps (DBLTs) were the first kind of optical trap realized by Arthur Ashkin and predate optical tweezers by two decades. Micron-sized dielectric objects, such as biological cells, are trapped by the combined scattering and gradient forces of two non-focused, counter-propagating Gaussian laser beams (see Figure 1). The main advantages of DBLTs are that (1) they can be simply realized by aligning two optical waveguides, such as single-mode optical fibers, (2) they can be combined with arbitrary imaging optics, and (3) the lack of focusing permits the use of high laser powers without cell damage ('opticution').

The latter aspect enables the controlled and nondestructive deformation of cells. When used for this purpose, DBLTs are called optical stretchers [7]. The cells' mechanical properties measured in this way have proven an important label-free marker of cell function, related to cancer progression, differentiation, cell cycle, infection etc. [4, 6]. The simplicity of the trap allows the straight-forward integration into microfluidic lab-on-chip environments [5] (see also Section 2). Microfluidic optical stretchers ( $\mu \mathrm{OS}$ ), thus, constitute a convenient technique to measure cell mechanics at rates up to 10 cells/min, which compares favorably with other standard ways of measuring cell mechanics, such as AFM, micropipette aspiration, where 10 cells/hour are more typical.

However, with the recent advent of really highthroughput (1,000 cells/sec) microfluidic techniques for the characterization of mechanical phenotypes that approach the analysis rates of conventional, fluorescence-based flow cytometers [9], the highthroughput aspect of the $\mu \mathrm{OS}$ has lost some of its former appeal. Instead, the current focus is shifting to the high-content investigation of cells, and also of other biological objects, which are temporarily, but arbitrarily long immobilized until several different physical parameters important for cell function have been determined in detail. For example, full mechanical characterization, in particular steady-state viscosity, which is particularly important to relate to cell migratory processes [4], takes at least several seconds per cell, and can inherently not be obtained in high-throughput, where the measurement time per cell is in the milliseconds range.

Other physical characteristics of interest are the refractive index (RI) of cells, which can be used to track cell differentiation and other functional changes

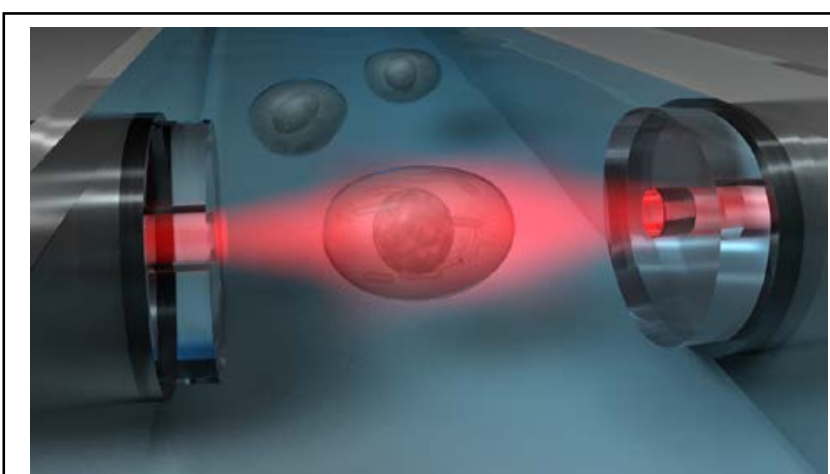

Figure 1 - Principle of microfluidic dual-beam laser traps. Cells are serially trapped between counterpropagating laser beams in a microfluidic channel.

[1], or their local mass density, which could be important for phase separations and phase transitions in cells. The choice of laser wavelength and total power also determines water absorption and with that the temperature in the volume of the trapped object [3]. By intentional use of this laser heating, additional thermodynamic properties of the trapped object can be investigated [2]. DBLTs can also be combined with other photonic techniques such as Raman and Brillouin spectroscopy and microscopy for additional chemical and mechanical information. Ideally, all these properties should be resolved in 3D inside of cells with diffraction-limited resolution so that either fast pointscanning, light-sheet or tomographic approaches become relevant. And finally, the investigation does not need to remain limited to entire cells. Also other, often rather delicate, biological objects can be gently handled, manipulated and investigated by optical forces in combination with photonic techniques. In this context, vesicles, isolated and artificial cell nuclei, mitotic spindles, or protein droplets come to mind.

\section{Current Status and Challenges}

In order to realize the potential of DBLTs to deliver high-content physical information about cells, as outlined above, and considering the state-of-the-art in the field, there are a number of challenges to be met.

The optical trapping itself [7], the extraction of detailed viscoelastic properties [4], and the microfluidic integration [5] has been well elaborated. The temperature increase in the trap was until recently coupled directly to the amount of power used, since DBLTs were conveniently realized with Ytterbiumdoped fiber-lasers operating at $1064 \mathrm{~nm}$. Here, $\Delta \mathrm{T}=13 \mathrm{~K} / \mathrm{W}$ [3]. By the recent advent of alternative high-power fiber-based lasers, such as frequencydoubled Erbium lasers, also $780 \mathrm{~nm}$ sources are now available, which reduces the temperature increase 8fold and permits the decoupling of optically induced stress and temperature [2]. 
The combination of DBLTs with many different conventional imaging techniques, such as phase contrast microscopy, is straight-forward. For fluorescence imaging, the limited usability of high-NA objectives - due to the significant distance between objective and trapped object, caused by the diameter of the optical fiber and the mounting on thick microscope slides - restricts the spatial resolution and light gathering ability of weaker fluorescence signals. While $\mathrm{Ca}^{2+}$ dyes and nuclear dyes can be readily imaged [2], sparser fluorescent signals from GFP-proteins or fainter signals from fluorescently labeled surface markers pose considerable challenges at present.

For quantitative phase imaging, often digital holographic microscopes are used. Their integration with DBTLs, set up on a commercial microscope stand, is less straight-forward especially when a separate reference beam needs to be implemented. From such quantitative phase information, assuming spherical symmetry, which is often given for cells in suspension, a 2D integrated RI distribution and an overall average refractive index can be calculated [10]. In order to obtain the complete $3 \mathrm{D}$ distribution of RI, and mass density, tomographic reconstruction has to be used. For this, phase images need to be obtained from multiple angles. Here, the versatility of DBLTs offers at least two attractive solutions: (1) either cells can be trapped by a conventional DBLT with two counterpropagating Gaussian laser beams with cylindrical rotational symmetry and then rotated by fluid flow in the microfluidic channel or (2) at least one of the Gaussian laser beams is replaced by a laser beam with higher-order modes that break the rotational symmetry, which leads to a preferential rotational alignment of the cell in the trap. The rotation of the laser modes then leads to the rotation of the trapped cell around the optical axis of the laser trap - and through the focal plane of the imaging microscope. When used for this latter purpose, DBLTs are also referred to as optical cell rotators [8] (see Figure 2). An important challenge in this context is the translational and rotational stability of the trap, currently limiting the spatial resolution of any imaging.

While Raman microscopy has been researched and described extensively, Brillouin microscopy of biological cells has only recently been introduced. It promises to yield additional mechanical information via interrogation of the local phononic spectrum inside cells. The extraction of actual mechanical properties requires knowledge of the local density and RI, which can be provided by the simultaneous RI tomography as described above. A significant challenge is the small frequency shift away from Rayleigh scattering and the very low intensity of the Brillouin signal, which leads to long integration times, amplifying the problem of

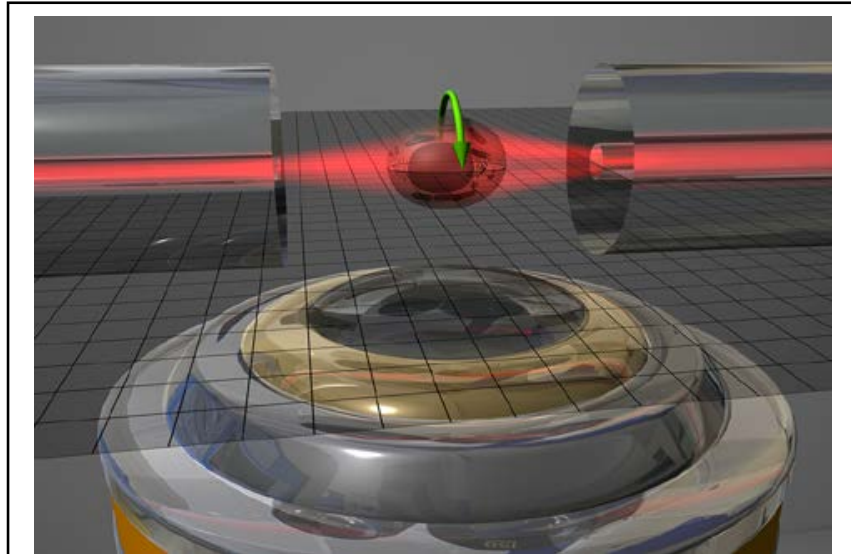

Figure 2 - Cell rotation in an optical cell rotator can be combined with any arbitrary microscopic or spectroscopic technique for full physical characterization.

translational and rotational stability of the object in the trap.

\section{Advances in Science and Technology to Meet Challenges}

The challenge of high-quality fluorescence imaging in DBLTs can be addressed by an optimized integration into specific lab-on-chip setups with the specific consideration of the optical quality along the imaging axis and the minimization of the distance between objective and trapped object [5] (see also Section 2). The use of more sensitive and faster fluorescence cameras, and an improved translational stability of the trap (see also below) will improve detection of weak signals and spatial resolution. The problem with incorporating quantitative phase microscopy can be solved by in-line interferometry, such as quadrilateral shearing interferometers. These are now commercially available, and can be mounted onto microscope stands just like other cameras.

The challenge of sufficient translational and rotational stability of DBLTs can be approached by fast feedback loops, involving real-time analysis of the position and 3D RI distribution of the trapped object and appropriate shaping of the light fields and their intensity using adaptive optics through the trapping fibers. The creation of complex light fields through multi-mode fibers using transfer-matrix operators has recently been demonstrated. This could also be used for illuminating cells with a light-sheet through the trapping fibers for improved fluorescence or even Brillouin microscopy. The problem with long integration times in Brillouin microscopy can potentially be ameliorated by stimulated Brillouin microscopy, and the use of line- or even light sheetimaging.

\section{Concluding Remarks and Perspectives}


In summary, microfluidic DBLTs are ideal "optical work benches" where cells can be temporarily held stationary, interrogated by a number of different photonic techniques for their complete physical phenotyping and then conveniently exchanged and sorted by the microfluidic integration. Even though their high-throughput mechanical phenotyping appeal has faded with the recent advent of techniques that are 100,000 times faster, their true potential lies in the high-content characterization and is still to be realized. The best times of microfluidic DBLTs are yet to come.

Acknowledgments - The author would like to thank R. Ananthakrishnan, L. Boyde, K. Chalut, C.J. Chan, G. Cojoc, U. Delabre, A. Ekpenyong, Ch. Faigle, J. Käs, M. Kreysing, F. Lautenschläger, B. Lincoln, P. Müller, S. Rönicke, S. Schinkinger, M. Schürmann, K. Travis, G. Whyte, and F. Wottawah for their essential contributions to the development of microfluidic DBLTs. Recent technological developments have been funded by the ERC Starting Investigator Grant "LightTouch" (\#282060). The artistic illustrations are provided by Effigos GmbH, Leipzig.

\section{References}

[1] Chalut KJ, Ekpenyong AE, Clegg WL, Melhuish IC and Guck J. 2012. Quantifying cellular differentiation by physical phenotype using digital holographic microscopy. Integrative Biology. 4 280-284.

[2] Chan CJ, Whyte G, Boyde L, Salbreux G and Guck J. 2014. Impact of heating on passive and active biomechanics of suspended cells. Interface Focus. 4 20130069-20130069.

[3] Ebert S, Travis K, Lincoln B and Guck J. 2007. Fluorescence ratio thermometry in a microfluidic dual-beam laser trap. Optics Express. 15 1549315499.

[4] Ekpenyong AE, Whyte G, Chalut KJ, Pagliara S, Lautenschläger F, Fiddler C, Paschke S, Keyser UF, Chilvers ER and Guck J. 2012. Viscoelastic Properties of Differentiating blood cells are fate- and functiondependent. PLoS ONE 7 e45237.

[5] Faigle C, Lautenschläger F, Whyte G, Homewood P, Martin-Badosa E and Guck J. 2015. A monolithic glass chip for active single-cell sorting based on mechanical phenotyping. Lab Chip 15 1267-1275.

[6] Guck J and Chilvers ER. 2013. Mechanics meets medicine. Sci Transl Med 5 212fs41.

[7] Guck J, Ananthakrishnan R, Mahmood H, Moon TJ, Cunningham CC and Käs J. 2001. The optical stretcher: a novel laser tool to micromanipulate cells. Biophys J 81 767-784.

[8] Kreysing M, Ott D, Schmidberger MJ, Otto O, Schürmann M, Martin-Badosa E, Whyte G and Guck J. 2014. Dynamic operation of optical fibres beyond the single-mode regime facilitates the orientation of biological cells. Nat Commun 5 5481-5486.
[9] Otto O, Rosendahl Ph, Mietke A, Golfier S, Herold Ch, Klaue D, Girardo S, Pagliara S, Ekpenyong A, Jacobi A, Wobus M, Töpfner N, Keyser UF, Mansfeld J, Fischer-Friedrich E, and Guck J. 2015. Real-time deformability cytometry: on-the-fly cell mechanical phenotyping. Nat. Methods 12 199-202.

[10] Schürmann M, Scholze J, Müller P, Chan CJ, Ekpenyong AE, Chalut KJ and Guck J. 2015. Refractive index measurements of single, spherical cells using digital holographic microscopy. Meth Cell Biol 125 143-159. 
12. Integration of fiber-based tweezers and microfluidic systems - Carlo Liberale

King Abdullah University of Science and Technology (KAUST)

\section{Relevance of the topic}

Over the last decades, microfluidic technologies have demonstrated their potential for the realization of labon-chip (LoC) systems. LoC devices present integration and miniaturization of various probes and devices, along with parallelization of functions and analyses, which allow cost-effectiveness (e.g. thanks to low consumption of samples and chemicals) and automated high throughput, that are particularly beneficial to biomedical applications. In this context, considerable interest is attracted by implementing Optical Tweezers (OT) in LoC devices, to support functions like precise positioning of samples within the micro-channel, single cell manipulation and sorting. Optical Tweezers are based on opto-mechanical forces, originated by exchange of momentum between photons and matter, that are exerted by a strongly focused optical beam on micro- or nano-particles [2]. They have been used in many applications in biology and physics, for a wide variety of experiments ranging from trapping and manipulation of live bacteria and cells, to cooling, trapping and manipulation of single atoms [2]. Important results have been achieved also by combining OT with other optical techniques, such as fluorescence and Raman spectroscopy. Early OT setups were based on tight focusing of a laser beam by means of high Numerical Aperture (NA) microscope objectives (usually $\mathrm{NA}>1$ )[2]. In order to overcome issues that arise in this scheme when trapping particles within thick samples or in turbid media, an effort to implement OT based on optical fibers has been in place since early 1990's. However, the realization of a fiberbased OT (FOT) forming three-dimensional optical traps has proven to be a challenging task, mainly because of the low NA of optical fibers. Indeed threedimensional optical trapping was initially demonstrated only with configurations made of at least two separated and opposing fibers. The early-proposed schemes based on a single fiber were essentially capable of only two-dimensional trapping, therefore were unable to stably trap particles in the middle of a fluid, or had impractically small trapping distances, making it difficult to trap a particle without physical contact. Only with more recent advances in technologies for microstructuring of the fiber tip it has become possible to design and fabricate OT based on single optical fibers. Fiber-based OT have recently been demonstrated, which present ready compatibility with microfluidic channels and are best suited towards realization of fully integrated microfluidic chips. Future developments of fiber-based OT for LoC

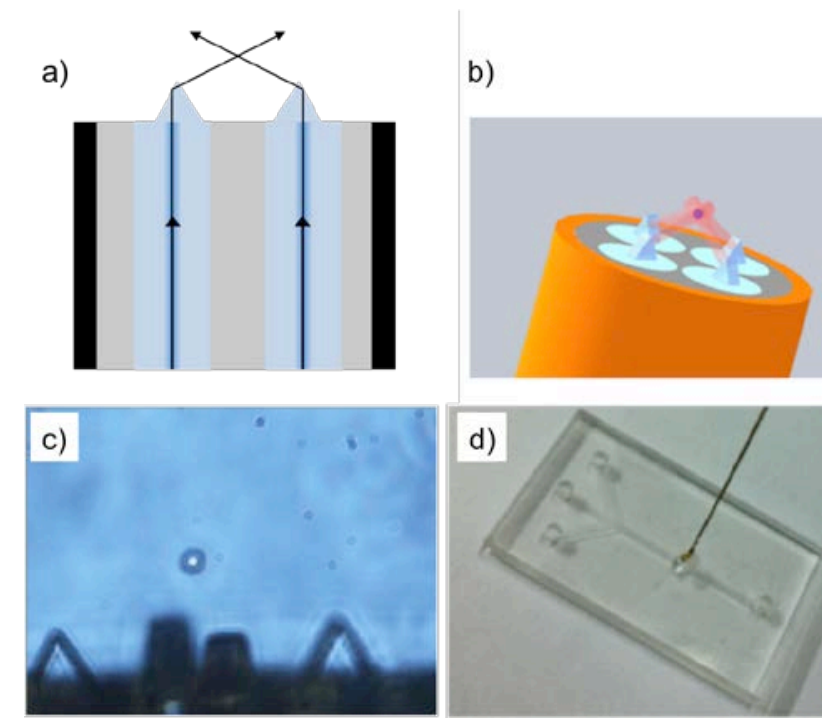

Figure 1 - (a) cross-section of the FOT probe presented in [1]: beams propagating in two symmetrically positioned fibres are reflected at the interface between microprisms and the outer medium; (b) isometric view of the FOT; (c) red blood cell trapped by the FOT in hypotonic solution; (d) picture of a microfluidic chip where the FOT has been inserted. Adapted with permission from [1].

devices could integrate, besides optical trapping, more advanced functions as imaging and/or enhanced spectroscopies with plasmonic probes.

\section{Current Status and Challenges}

At the state of the art, three-dimensional optical traps are mainly implemented inside microfluidic devices by using three different approaches: 1) with high Numerical Aperture (NA) microscope objective-based setups; 2) by two counter-propagating beams, delivered by two optical waveguides (which could be optical fibers) positioned on opposite sides of the microfluidic channel; 3) by single optical fibers. Naturally only the two latter methods bring capability for integration into microfluidic chips, and are then compatible with the development of portable LoC devices. Optical Tweezers based on two opposed waveguides are also called "optical stretchers" and are mainly used to apply forces that deform the trapped particle, to probe its mechanical properties [3]. This particular type of integrated OT is considered in Section 11 to this roadmap article.

Integrated OT based on a single optical fiber, able to generate a three-dimensional optical trap far enough from the fiber tip to avoid contact with the sample, are currently possible only by the use of specialty fibers (bundles, multicore or annular core fibers) along with advanced methods to modify the fiber tip to overcome its intrinsic low NA [4]. Remarkably, the continuous progress made in the capability to structure the fiber tip has enabled ever more effective geometries for optical trapping. Indeed, different fiber tip modification 
methods have been proposed so far. Initially, tapering of a standard single mode optical fiber to a very small tip size with the heating and pulling method, resulted in a FOT with a very small trapping distance [5]. More recently, high-precision fabrication techniques were used to implement an approach proposed in [6], where light beams having an annular arrangement (e.g. generated by an annular core fiber or carried by the different fibers of a bundle) are deflected by Total Internal Reflection (TIR) towards a common crossing point, yielding the equivalent effect of a high-NA microscope objective, as needed for OT (figure 1(a)(b)). The TIR deflecting structures have been fabricated on the fiber tip by Focused Ion Beam milling [6] or, more recently, by Two-PhotonLithography (TPL) (figure 1(c)) [1]. The latter fabrication method is significantly easier, more flexible and cost-effective than the former. The use of a fiber bundle instead of an annular fiber allows specialization of different subsets of fibers for optical trapping and for simultaneous probing of the trapped sample (e.g. with fluorescence). A similar scheme, based on fiber annular core distribution and TIR-based deflection, has been realized also by the grinding an polishing technique [7].

As shown in [1], the single-fiber based OT can be integrated into a microfluidic device (figure 1(d)), in a vertical configuration that would allow to insert multiple FOT at different locations within the same LoC device without interfering with the microfluidic channel network. Trapping within microfluidic channels has also been lately demonstrated with a tapered fiber creating a two-dimensional trap, under specific flow rate conditions, [8] and with a tapered fiber for three-dimensional trapping but with the particle in contact with the tip [9].

A few challenges still remain towards the goal of bringing full functionalities of well-developed "bulk" OT in LoC devices. A first challenge is to allow all optical manipulation (translation in three dimensions, rotation) of trapped samples without moving the FOT probe. A second challenge is the creation of multiple optical traps with a single FOT within a microfluidic channel. Further challenges will be the addition of imaging capabilities to the FOT probe, e.g. by integrating multimode fibers [10], and of enhanced probing/sensing capabilities, e.g. with electrodes or plasmonic based structures.

\section{Advances in Science and Technology to Meet Challenges}

The addition of more advanced functionalities to single fiber FOT, for integrated LoC devices, will be possible with the progress on design and fabrication of complex photonic structures on the tip of optical fibers. In this regard, a key role of the TPL fabrication method is envisioned, as an extremely powerful tool for rapid and flexible prototyping of high optical quality elements, with dimensions that range from few to hundreds of microns. In particular, a development of a new design that further reduce the size of single fiber FOT will improve compatibility with small microfluidic channels and will allow an easier integration of other fibers, e.g. a multimode fiber for imaging, and/or other structures, e.g. for enhanced spectroscopy, within the FOT probe, to obtain an ultra-compact multimodal tool for LoC devices. Another important advance will be the combination with other technologies that could enable new functions, as the use of Spatial Light Modulators for fiber imaging [10] or towards creation of dynamical multiple traps, which will also allow manipulation of the trapped particle.

\section{Concluding Remarks and Perspectives}

Integration of Optical Tweezers in microfluidic channels will enable new functions in LoC devices. Optical fibers offer an excellent platform for miniaturization of OT, so that they can be embedded in microfluidic circuits. Indeed single-fiber FOT have been recently reported, based on specialty fibers and TIR structures, and inserted in a microfluidic channel. Future developments will concern: further miniaturization of the FOT; addition of manipulation and multi-trap capabilities; integration of new functionalities, like imaging and enhanced spectroscopies, to the FOT probe.

Acknowledgments - The author would like to thank the following current and former colleagues for their valuable contributions in the joint work on the subject: Francesca Bragheri, Patrizio Candeloro, Gheorghe Cojoc, Ilaria Cristiani, Enzo Di Fabrizio, Lorenzo Ferrara, Paolo Minzioni, Gerardo Perozziello and Vijayakumar P. Rajamanickam.

\section{References}

[1] Liberale C, Cojoc G, Bragheri F, Minzioni P, Perozziello G, La Rocca R, Ferrara L, Rajamanickam V, Di Fabrizio E and Cristiani I 2013 Integrated microfluidic device for single-cell trapping and spectroscopy Scientific Reports 3:1258

[2] Ashkin A, Optical trapping and manipulation of neutral particles using lasers. World Scientific: Singapore ; Hackensack, NJ, 2006; p xxiv, 915 p.

[3] Yang T, Bragheri $F$ and Minzioni P 2016 A Comprehensive Review of Optical Stretcher for Cell Mechanical Characterization at Single-Cell Level Micromachines 7:5

[4] Ribeiro R S R, Soppera O, Oliva A G, Guerreiro A and Jorge P A S 2015 New Trends on Optical Fiber Tweezers $J$ Lightwave Technol 33 3394-3405 
[5] Liu Z H, Guo C K, Yang J and Yuan L B 2006 Tapered fiber optical tweezers for microscopic particle trapping: fabrication and application Optics Express 14 12510-12516 [6] Liberale C, Minzioni P, Bragheri F, De Angelis F, Di Fabrizio E and Cristiani I 2007 Miniaturized all-fibre probe for three-dimensional optical trapping and manipulation Nature Photonics 1 723-727

[7] Zhang Y, Liu Z H, Yang J and Yuan L B 2012 A noncontact single optical fiber multi-optical tweezers probe: Design and fabrication Optics Communications 285 40684071

[8] Gong Y, Zhang C L, Liu Q F, Wu Y, Wu H J, Rao Y J and Peng G D 2015 Optofluidic tunable manipulation of microparticles by integrating graded-index fiber taper with a microcavity Optics Express 23 3762-3769

[9] Xin H B, Li Y Y, Li L S, Xu R and Li B J 2013

Optofluidic manipulation of Escherichia coli in a microfluidic channel using an abruptly tapered optical fiber Applied Physics Letters 103:033703

[10] Morales-Delgado E E, Psaltis D and Moser C 2015 Two-photon imaging through a multimode fiber Optics Express 23 32158-32170 


\section{Acoustic prefocusing in optofluidic systems - Kirstine Berg-Sørensen Technical University of Denmark \\ Relevance of the topic}

The ability of acoustic fields to position small particles was discussed already by Ernst Florens Friedrich Chladni (1756-1827), and is familiar to many scholars through the demonstration experiment named "The Chladni plate". When carried over to dimensions of typical microfluidic systems, the resonance phenomenon nicely illustrated by the Chladni plate experiment, implies typical frequencies in the $\mathrm{MHz}$ range. Many research papers have in recent years demonstrated the ability of the effect, termed acoustophoresis, e.g. to sort cells according to their acoustic properties; readers unfamiliar with the topic may consult the tutorial series of papers in Lab-onChip [1]. The second part of the topic, optofluidic systems, is the term currently used for microfluidic systems with embedded optical elements, be it a microlaser, or a passive or active optical element; the relevance of optofluidic systems at large is demonstrated by the collection of papers in the current roadmap on Optofluidics. The benefits of a combination of the effects of acoustic and optical manipulation has been illustrated by the ability for active particle sorting [2], improved trapping efficiency in an optical stretcher setup [3] and access to complementary mechanical characteristics for the same single cancer cell [4]. Acoustic prefocusing in an optical stretcher setup is illustrated in Figure 1. The results of references [2-4] suggest that further advances in the field may find both technological and biomedical applications.

\section{Current Status and Challenges}

The combination of acoustofluidics and optical manipulation is, to the best of the author's knowledge, only applied in very few labs [2-4], with promising recent advances involving high-resolution imaging [5]. As discussed below, one may very well imagine inclusion of other characterization tools based on optics.

On the acoustics side, an interesting development is the recent introduction of the concept of iso-acoustic properties, whereby the understanding and control in acoustic focusing and sorting has been tremendously widened [6]. A current challenge for the technology to be more accessible for applications is the choice of material for the construction of the microfluidic systems. The successful demonstrations of combined acousto- and optofluidics [2-5] are based on glass - a hard and highly resistant material with excellent optical properties. Yet, much current research explores the

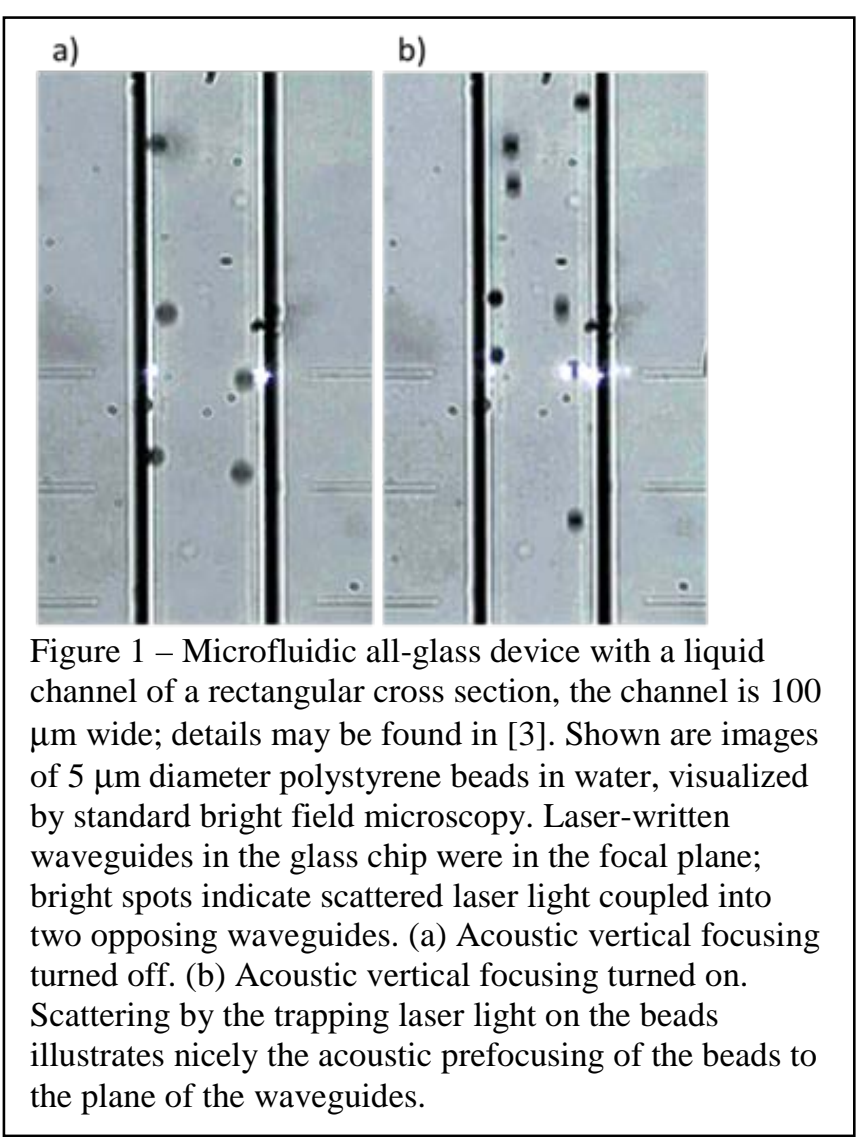

ability to construct micro- and optofluidic systems in polymer materials since micro- and even nanofluidic systems may be produced by large-scale production techniques such as injection molding or roll-to-roll imprinting rather than the expensive and timeconsuming clean-room processes required for the production of a single microfluidic system in glass [7]. In addition, the polymer material can be chosen with properties that match well both optical and biomedical requests. Unfortunately, acoustofluidics with plastic materials remain challenging due to the relatively low sound speed and acoustic impedance in most of the transparent plastics. This implies that acoustic energy may be transferred to, and lost in, the device itself, and that knowledge of the conditions for acoustic resonance, leading to large acoustic forces, requires detailed 3D modelling of the pressure fields in the entire device [8]. Such detailed models are in contrast to simple $1 \mathrm{D}$ models in which one assumes a large degree of reflection of the sound wave at the interface between the liquid in the microfluidic channel and the hard walls of the glass or silicon microfluidic device.

As discussed in section 3, lithium niobate offers an attractive alternative choice of material.. In [5], a transparent transducer made out of $\mathrm{LiNbO}_{3}$ with indium tin oxide (ITO) electrodes was applied, in a layered device. The experimental setup was 
demonstrated to be able to provide precise determination of both optical and acoustic forces on trapped silica microspheres.

Delivery or collection of light for optical manipulation or characterization by optical means is another point to decide on. One option is to have the light delivered by embedded optical fibers [9], or waveguides directly written into the microfluidic device [3-4], suitable for optical traps based on two counter propagating laser beams, or for collection of emitted light, e.g. for Raman spectroscopy of the sample. Another option, relevant for single beam optical traps, is to rely on light delivery and light collection through an external microscope objective [2,5]. When this latter option is chosen, one should be aware that the acoustic force field may be altered due to the mechanical coupling between the immersion oil to the microscope objective and thus the entire microscope [5].

\section{Advances in Science and Technology to Meet Challenges}

As alluded to above, on the materials side, the combination of acoustophoresis and optofluidics would clearly benefit from the development of transparent polymer materials with larger acoustic contrast between device material and liquid - in addition to requests of good optical quality and high chemical resistance. As this may not be realistic, the second best is to develop and apply 3D modelling tools [8] that enables prediction of the sound wave propagation in the entire experimental device and thereby may suggest a design of the microfluidic device optimized for the simultaneous action of acoustic and optical forces.

The choice of a polymer material for the microfluidic device couples well with a desire to develop single use devices. Waveguides produced by post-processing steps like DUV exposure and/or other means to embed optical elements in a polymer chip produced by massproduction techniques would clearly enhance the employability of optofluidic devices, with or without acoustophoresis. A challenge to resolve is, however, the typical loss of optical power of order 0.5-0.7 $\mathrm{dB} / \mathrm{mm}$ in waveguides written by DUV or direct laser writing, in addition to the coupling losses into a square or rectangular waveguide. Alternatively, light coupling through standard optical fibers suffers much less from loss of optical power, and still offers a competitive solution although manual assembly is required. With either choice of solution for light propagation in the microfluidic chip, the dream device would be a single use polymer device that is to be mounted in a system or a holder that includes efficient and well-aligned coupling to light sources as well as an efficient coupling to a sound transducer. In this respect, piezoceramic crystals may still be a wise choice for the

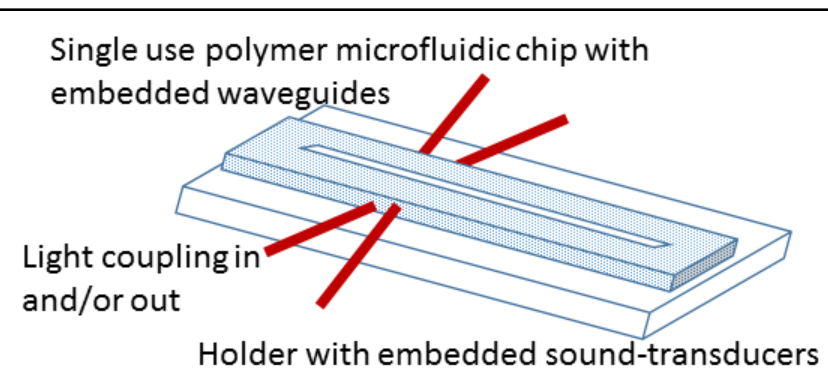

Figure 2 - Simple sketch of a future device for simultaneous acoustic prefocusing, optical manipulation and spectroscopic characterization.

acoustic transducer, but capacitive micromachined ultrasound transducers, which may even be controlled by light, may be an interesting alternative [10].

\section{Concluding Remarks and Perspectives}

As discussed, manipulation by both sound and light fields in microfluidic systems adds versatility to the optofluidics toolbox. The text concentrated on merging tools of manipulation applying both optical and sound waves, yet the perspectives include other uses of light, e.g., for spectroscopic characterization. One may foresee a combination of optical manipulation and/or spectroscopy on cells with particular iso-acoustic properties, with the perspective to learn many more details, including molecular, about the properties of such individual cells.

\section{Acknowledgments -}

The author acknowledges discussions over the years with G. Nava, T. Yang, P. Minzioni, I. Cristiani, as well as with A. Kristensen and with $\mathrm{H}$. Bruus and his students. Financial support from the Carlsberg Foundation and COST MP1205 is highly appreciated.

\section{References}

[1] Tutorial series on acoustofluidics (2011): Acoustofluidics-exploiting ultrasonic standing wave forces and acoustic streaming in microfluidic systems for cell and particle manipulation Lab Chip 11 3579-3580

[2] G Thalhammer, R Steiger, M Meinschad, M Hill, S Bernet, and M Ritsch-Marte (2011): Combined acoustic and optical trapping Biomed Opt Exp 2 2860-2870

[3] G Nava, F Bragheri, T Yang, P Minzioni, R Osellame, I Cristiani, and K Berg-Sørensen (2015): All-silica microfluidic optical stretcher with acoustophoretic prefocusing Microfluid Nanofluid 19 837-844

[4] T Yang, F Bragheri, G Nava, I Chiodi, C Mondello, R Osellame, K Berg-Sørensen, I Cristiani, and P Minzioni (2016): A comprehensive strategy for the 
analysis of acoustic compressibility and optical deformability on single cells Sci Rep 623946

[5] G Thalhammer, C McDougall, MP MacDonald, and M Ritsch-Marte (2016): Acoustic force mapping in a hybrid acoustic-optical micromanipulation device supporting high resolution optical imaging Lab Chip 16 1523-1532

[6] P Augustsson, JT Karlsen, H-W Su, H Bruus, and J Voldman (2016): Iso-acoustic focusing of cells for size-insensitive acousto-mechanical phenotyping Nature Comm 711556

[7] H Becker and LE Locascio (2002): Polymer microfluidic devices Talanta 56 267-287

[8] J Dual and D Möller (2012): Acoustofluidics 4: Piezoelectricity and application in the excitation of acoustic fields for ultrasonic particle manipulation Lab Chip 12 506-514

[9] B Lyncoln, S Schinkinger, K Travis, F Wottawah, S Ebert, F Sauer, and J Guck (2007): Reconfigurable microfluidic integration of a dual-beam laser trap with biomedical applications Biomed Microdevices DOI 10.1007/s10544-007-9079-x

[10] B T Khuri-Yakub and Ö Oralkan (2011): Capacitive micromachined ultrasonic transducers for medical imaging and therapy J. Micromech Microeng. 21 054404 


\section{Optofluidics for single-cell protein analysis - Jian Chen \\ Institute of Electronics, Chinese Academy of Sciences}

\section{Relevance of the topic}

Proteins (i.e., macromolecules composed of chains of amino acid residues) perform key functions within organisms, including regulation of metabolic reactions, DNA replication, stimuli responsiveness, and molecule transportations [1]. As key components of biological organisms, quantities and activities of proteins have been regarded as the most important indicators of biological activities, which are closely related to phenomena such as cellular differentiation, neuron transmission and disease progression [2].

Within the last few decades, a large number of characterization approaches (e.g., gel electrophoresis, immunoassay, chromatography and mass spectrometry) have been developed to estimate the quantities and activities of proteins [3]. These results have contributed to the rapid developments of biological and medical sciences, which have significantly promoted the detection capabilities in clinical diagnosis [4]. However, these approaches are only capable of quantifying protein expressions from a large number of cells, which neglects the fact that most of the organisms are composed of cells with variations in their functionalities and thus protein expressions at the population level may mask key underlying mechanisms due to cellular heterogeneity.

Currently, fluorescent flow cytometry is the golden standard in the field of single-cell protein analysis where single cells mixed with fluorescence labeled antibodies are flushed into a fluorescent measuring domain composed of laser excitation and photomultiplier tube based fluorescent detection [5]. Based on this methodology, variations in single-cell protein expressions are closely correlated with tumor biology, microbiology, cellular differentiation and drug development (see Figure 1). However, fluorescent flow cytometry can only provide an absolute quantitation of surface proteins of single cells, while it cannot quantify intracellular proteins due to the lack of calibration approaches, and thus it cannot translate raw fluorescent signals into copy numbers of intracellular proteins [6].

Optofluidics is the science and technology on the processing and optical detection of small amounts of fluids $\left(10^{-9}\right.$ to $10^{-18}$ liters) in channels with dimensions of tens of micrometers [7]. Since the dimensions of optofluidics are comparable to biological cells, it has been used for the analysis of single-cell protein expressions [8], which can be classified into four major

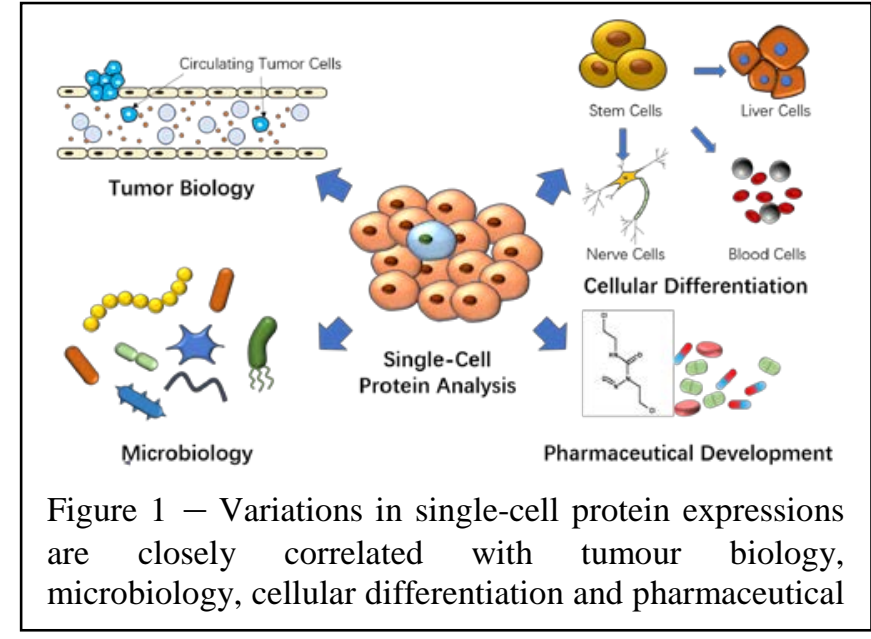

types: (1) optofluidic fluorescent flow cytometry; (2) droplet based optofluidic flow cytometry; (3) largearray micro wells (microengraving) and (4) large-array micro chambers (barcode micro chips). In this section, we examine the advantages and limitations of each technique and discuss future research opportunities by focusing on two key parameters: absolute quantification and throughput.

\section{Current Status and Challenges}

In optofluidics, the fluorescent micro flow cytometry is the first approach to quantify single-cell protein expressions where single cells are mixed with fluorescence labeled antibodies are flushed into microfabricated flow channels with fluorescent intensities measured [9] (see Figure 2(a)). As a miniaturized version of conventional fluorescent flow cytometry, the micro flow cytometry is featured with low sample requirement of cells and reagents. However, this approach cannot quantify the copy number of intracellular proteins, due to the lack of effective calibration approaches.

In the second approach, droplet based optofluidic flow cytometry was proposed where single cells, functionalized capture beads and fluorescence-labeled secondary antibodies are encapsulated in droplets, where micro beads bind cytokines secreted by single cells, which further bind fluorescence-labeled secondary antibodies, leading to fluorescent signals [10] (see Figure 2(b)).

Compared to conventional fluorescent flow cytometry, this approach can effectively assay secreted proteins at the single cell line. However, due to the use of cytokine-capture beads, there is an uneven distribution of fluorescence within individual droplets (intensity peaks around individual beads), which poses obstacles in calibration and thus the secreted proteins cannot be effectively quantified. 
In the third approach, large-array micro wells ("microengraving") were proposed to isolate individual cells with copy numbers of secreted proteins quantified [11]. As shown in Figure 2(c), single cells suspended in media are deposited into a large array of microwells, which are then sealed by a glass slide coated with a specific capture reagent. After certain periods of incubation, the glass slide is removed and further interrogated with laser-based fluorescence scanners. Although microengraving enables absolute quantification of proteins secreted by individual cells, compared to flow cytometry, it suffers from complicated processes and limited throughput

In the fourth approach, large-array micro chambers (single-cell barcoding microchips) were proposed to assay both cytosolic and membrane proteins of single cells [12]. As shown in Figure 2(d), the single-cell barcoding microchips consist of thousands of individually addressed micro chambers for single-cell trapping, followed by cell lysis, capture of targeted proteins by pre-printed antibodies on the surface of the chambers, which are further measured using immunosandwich assays. This approach is the most powerful optofluidic system in the field of single-cell protein analysis since it enables the absolute quantification of both surface and cytosolic proteins of single cells. However, since this approach relies on the confinement of single cells within chambers, it cannot be easily scaled up to further increase the throughput.

\section{Advances in Science and Technology to Meet Challenges}

Absolute quantification is a key requirement for singlecell protein assays since, without the capabilities of absolute quantification, the protein levels measured by different approaches cannot be effectively compared. From this perspective, new calibration approaches have to be developed in fluorescent micro flow cytometry, enabling the translation of raw fluorescent signals to protein copy numbers.

Throughput is also a key factor in single-cell protein quantification. Although optofluidic large-array devices are featured with parallel analysis of single cells, which are capable of processing hundreds of single cells within one experiment, they still cannot be used to process cell samples with heterogeneity (e.g., tumor samples with at least 1 million cells) due to their limited throughputs. Thus, tremendous efforts have to be devoted to this field with the purpose of further scaling up these assays. As to micro flow cytometry, further technical improvements may focus on the extension of this serial approach to function in parallel.
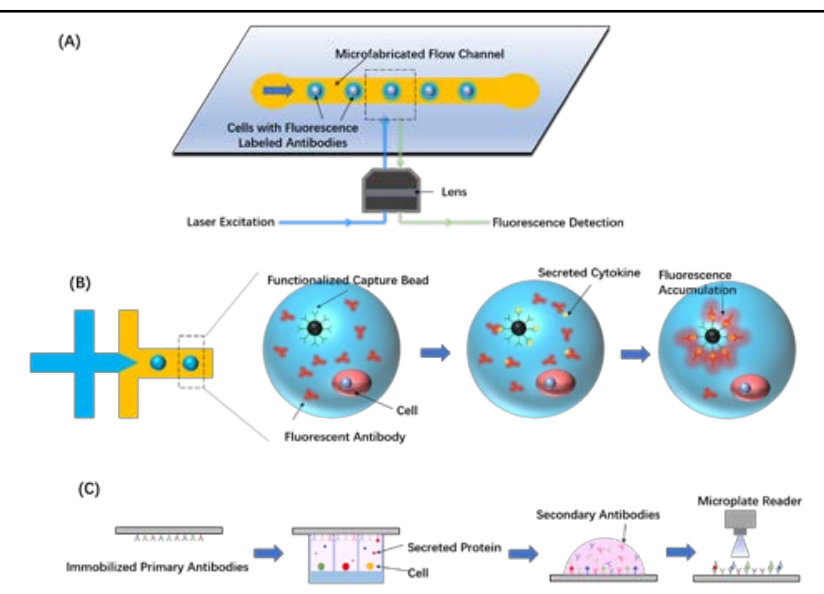

(D)

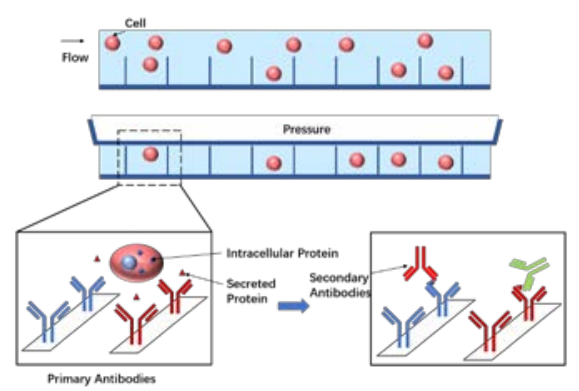

Figure 2 - Key developments of optofluidic systems enabling high-throughput single-cell protein characterization, which includes (a) optofluidic fluorescent flow cytometry; (b) droplet based optofluidic flow cytometry; (c) large-array micro wells (microengraving) and (d) large-array micro chambers (barcode micro chips).

\section{Concluding Remarks and Perspectives}

In this study, we summarize key developments of optofluidic platforms enabling the quantification of single-cell proteins. Although significant improvements have been made within the last decade, an ideal tool of single-cell protein quantification featured with absolute quantification and high throughput is still not available.

Acknowledgments - This work was supported by the National Basic Research Program of China (973 Program, Grant No. 2014CB744600), National Natural Science Foundation of China (Grant No. 61431019, 61671430), Key Projects of Chinese Academy of Sciences (QYZDB-SSW-JSC011), Natural Science Foundation of Beijing (4152056), Instrument Development Program of the Chinese Academy of Sciences, and Beijing NOVA Program of Science and Technology.

\section{References}

1. Kim, M.S., et al., A draft map of the human proteome. Nature, 2014. 509(7502): p. 575-81. 2. Savage, N., Proteomics: High-protein research. Nature, 
$2015 . \quad$ 527(7576): $\quad$ p. $\quad$ S6-7.

3. Phizicky, E., et al., Protein analysis on a proteomic scale. Nature, 2003. 422(6928): p. 208-15. 4. Hanash, S., Disease proteomics. Nature, 2003. 422(6928): $226-32$. 5. Janes, M.R. and C. Rommel, Next-generation flow cytometry. Nature Biotechnology, 2011. 29(7): p. 602-4. 6. Zenger, V.E., et al., Quantitative flow cytometry: interlaboratory variation. Cytometry, 1998. 33(2): p. 138-45. 7. Psaltis, D., S.R. Quake, and C. Yang, Developing optofluidic technology through the fusion of microfluidics and optics. Nature, 2006. 442(7101): p. 381-6.

8. Fan, B., et al., Development of Microfluidic Systems Enabling High-Throughput Single-Cell Protein Characterization. Sensors, 2016. 16(2): p. 232. 9. Chan, S.D., et al., Cytometric analysis of protein expression and apoptosis in human primary cells with a novel microfluidic chip-based system. Cytometry A, $2003 . \quad$ 55(2): $119-25$. 10. Huebner, A., et al., Quantitative detection of protein expression in single cells using droplet microfluidics. Chemical Communications, 2007(12): p. 1218-1220. 11. Han, Q., et al., Polyfunctional responses by human T cells result from sequential release of cytokines. Proceedings of the National Academy of Sciences of the United States of America, 2012. 109(5): p. 1607-12. 12. Ma, C., et al., A clinical microchip for evaluation of single immune cells reveals high functional heterogeneity in phenotypically similar T cells. Nature Medicine, 2011. 17(6): p. 738-43. 


\section{Optofluidics for DNA analysis - Markus} Pollnau $\mathrm{KTH}-$ Royal Institute of Technology

\section{Relevance of the topic}

Capillary electrophoresis (CE) is a powerful method for biomolecule separation and analysis. The sorting and sizing of DNA molecules within the human genome project [1] has been enabled largely by CE separation and analysis. The human genome project has also lead to the genetic mapping of various human illnesses [2]. The traditional techniques, e.g. using a bulk glass capillary filled with agarose gel [3], provide a high separation resolution, but typically require rather long analysis times and bulky instrumentation.

These issues are addressed by the development of microchip CE. Integration of a lab on a chip, thereby ensuring compactness and introducing the potential for mass fabrication, paves the way for device portability and in-field or point-of-care applications. Furthermore, DNA sequencing by microchip CE allows for cheap, high-speed analysis of low reagent volumes. In recent years on-chip integration of DNA sequencing [4] and genetic diagnostics [5] have become feasible. One of its potential applications is the identification of genomic deletions or insertions associated with genetic illnesses.

\section{Current Status and Challenges}

Laser-induced fluorescence exploiting fluorescent dye labels is the most popular microchip CE monitoring technique. Conventional instruments use confocal setups to focus the excitation light and to collect the resulting fluorescence. Such schemes can provide high sensitivity down to a detection limit of $200 \mathrm{fM}$ [6], corresponding to merely eight molecules in the excitation volume, making plausible the vision of going down to interrogating at the single-molecule level. However, these schemes require accurate mechanical alignment of the optics to the microfluidic channels and are sensitive to mechanical vibrations and drifts. The need to use microchip CE in combination with a massive benchtop instrument, such as an optical microscope, frustrates many of the microchip CE advantages, in particular it strongly limits device portability and prevents in-field or point-of-care applications. In the future optical components, including light generation, transportation, interaction with the reagents, collection and detection, need to be integrated, partly directly on the optofluidic chip.

Sizing accuracy poses an inherent challenge to CE and particularly microchip CE and achieving sufficient and reliable sizing accuracy for the envisaged DNA analysis is probably the biggest hurdle to be taken. Application of CE-based DNA sequencing in a lab-on- a-chip to identify genomic deletions or insertions associated with genetic illnesses, such as breast cancer or anemia, critically depends on the detection of single base-pair insertions or deletions from DNA fragments in the diagnostically relevant range of $150-1000$ basepairs. The sizing accuracy is related to DNA plug formation and depends on many factors, including the range of DNA fragment sizes to be separated, the choice of - and the changes in - the sieving gel matrix inside the microfluidic channel and its inner-wall coating, temperature and actuation voltage, as well as the choice of microfluidic channel cross section, dye label and, finally, the calibration procedure. A number of empirical studies have been performed to shed light on this complex situation, but results are sometimes not reproducible and existing models are typically inaccurate and unable to predict device performance.

\section{Advances in Science and Technology to Meet Challenges}

With the general advancement in micro-fabrication, significant technical progress has been made also in merging microfluidics and micro-optics in an optofluidic chip. For example, post-processing of commercial microfluidic chips by femtosecond laser writing of optical waveguides [7, 8] that can direct laser light to the interaction point and/or collect fluorescence from the excited dye labels and deliver it to the detection unit is a suitable method for optofluidic integration. This technique has the additional capability of writing three-dimensional optical structures into the microfluidic chip [9]. The light source and the detection unit are preferably placed in a small portable

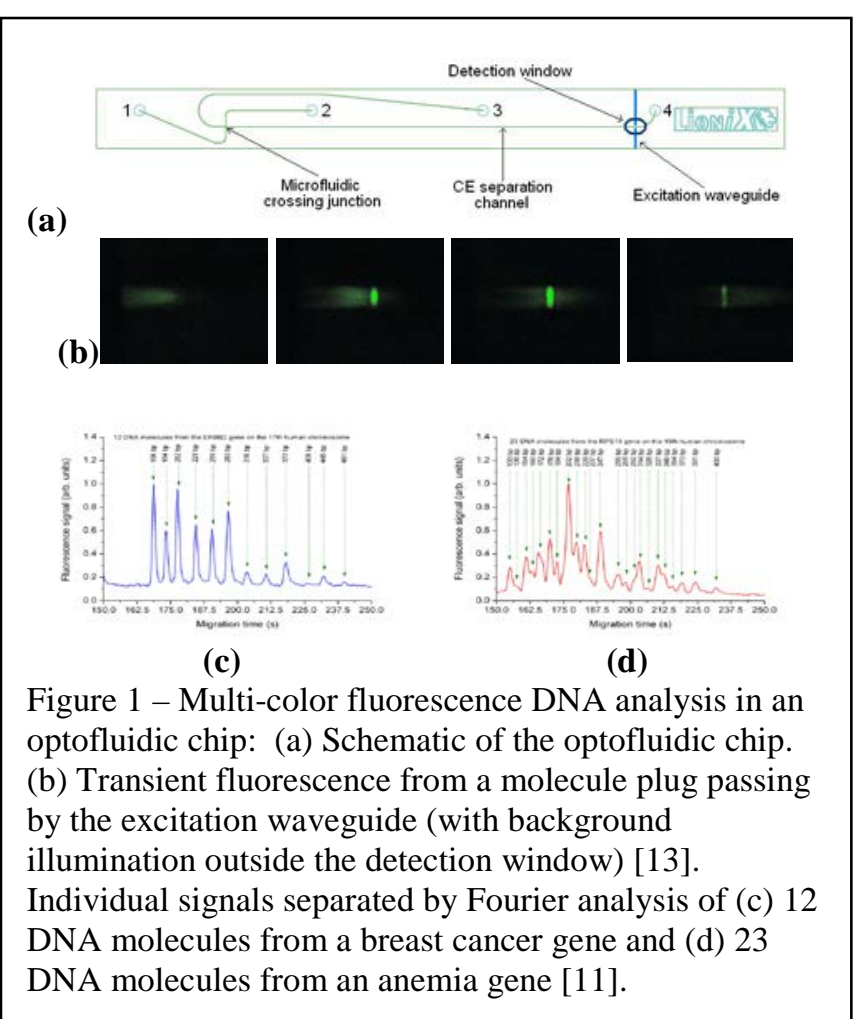


instrument that also includes the steering unit for the actuation voltages and to which the optofluidic chip can be precisely attached.

The technique of multiplex ligation-dependent probe amplification [10] allows for the simultaneous extraction and individual end-labeling of DNA fragments from independent human genomic segments. In order to exploit this technique, parallel optical processing of the prepared sample is required. A demonstrated parallel optical processing method is modulation-frequency-encoded dual-wavelength laser excitation, fluorescence detection with a single ultrasensitive, albeit color-blind photomultiplier, and Fourier analysis decoding [11, 12], see Fig. 1. To make the laser excitation of dye labels selective, each label must absorb light in a different wavelength range. Typical dye labels exhibit rather large absorption bandwidths, thereby strongly limiting the number of exclusive labels that can be employed simultaneously. Consequently, the extension of dual-wavelength analysis to a larger number of wavelengths faces the challenge of development of novel, narrow-excitationband fluorescent labels. Alternatively, fluorescent labels with spectrally overlapping absorption bands can be excited with a single laser, thereby reducing the necessary infrastructure on the excitation side, and their individual fluorescence lines can be either detected by a color-sensitive detector array or spectrally dispersed and then detected by a detector array. However, any complication on the detection side that wastes fluorescence intensity or provides less sensitivity than a photomultiplier impairs the detection limit.

The dependence of sizing accuracy on the important system parameters must be investigated more systematically and its theoretical background needs to be improved. For example, recently it has been identified that the choice of fluorescent dye label can significantly influence the sizing accuracy and that a proper choice can largely improve the accuracy [14], see Fig. 2, but the reason for this effect is unknown and systematic investigations are lacking. In the same work it has been demonstrated that the calibration strategy influences the sizing accuracy [14]. On the one hand, it may not matter much whether the optofluidic device is calibrated by flow of a known reference sample in the same or in a separate experiment and whether the DNA fragments of the reference sample are almost identical or slightly different from those of the unknown sample to be investigated. On the other hand, applying the same fluorescent dye label to the two samples appears to be crucial.

Most importantly, the dependence of migration time on base-pair size needs to be understood theoretically. The results displayed in Fig. 2 prove that a quadratic fit

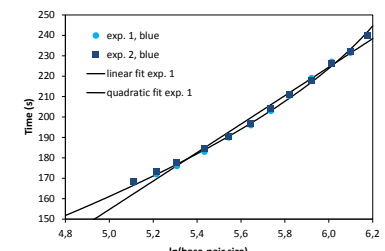

(a)

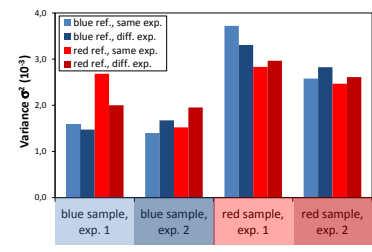

(c)

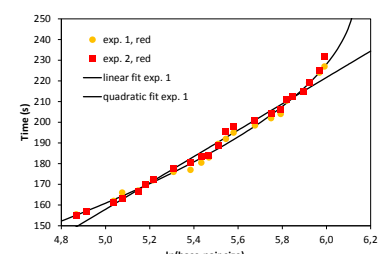

(b)

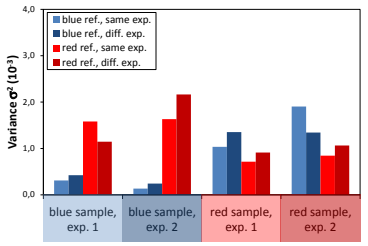

(c)
Figure 2 - Migration time (linear scale) versus base-pair size (logarithmic scale) of (a) 12 blue-labeled and (b) 23 red-labeled DNA molecules simultaneously migrated and separated in experiment 1 (circles) and experiment 2 (squares) [14]. Linear and quadratic fits (solid lines) shown only for experiment 1 . Variance $\sigma^{2}$ measured in experiment 1 or 2 from (c) the linear fit and (d) the quadratic fit, obtained from the measured reference data from the same or the different experiment [14].

reproduces the measured data significantly better than a linear fit, indicating that the assumption of a simple logarithmic dependence of migration time on base-pair size underlying the production of DNA ladders with a logarithmic increase in base-pair size is not justified by the experiment [14]. Nevertheless, the sizing accuracy crucially depends on the applied law to fit the reference data. At present, in the absense of a proper theoretical understanding of DNA plug formation and flow mechanism the choice of fit function is merely a best guess and may even change with the underlying system parameters.

Choice of a suitable dye label, combined with reference calibration and sample investigation by fluorescent detection in consecutive experiments and application of a best-guess fit function, has resulted in capillary electrophoretic separation of fluorescentlabeled DNA molecules in the 150-1000 base-pair range with single-base-pair resolution [14], see Fig. 2, thereby paving the way towards the detection of single base-pair insertion or deletion in a lab-on-a-chip with low reagent volumes in a few-minute experiment.

\section{Concluding Remarks and Perspectives}

Through the efforts of many scientists in this research area, in-field and point-of-care applications have become feasible, but a considerable amount of work remains to be performed to better understand the dependence of sizing accuracy on the various system parameters by establishing a better theoretical framework and to improve the measurement repeatability and reliability. 
Acknowledgments - The author gratefully acknowledges collaboration with his co-workers C. Dongre and H. J. W. M. Hoekstra, the Politecnico di Milano, LioniX B.V. and Zebra Bioscience B.V.

\section{References}

[1] International Human Genome Sequencing Consortium 2001 Initial sequencing and analysis of the human genome Nature $\mathbf{4 0 9}$ 860-921

[2] Altshuler D, Daly MJ and Lander ES 2008 Genetic mapping of human disease Science 322 881-888

[3] Brody JR and Kern SE 2004 History and principles of conductive media for standard DNA electrophoresis Anal. Biochem. 333 1-13.

[4] Fredlake CP, Hert DG, Kan CW, Chiesl TN, Root BE, Forster RE and Barron AE 2008 Ultrafast DNA sequencing on a microchip by a hybrid separation mechanism that gives 600 bases in 6.5 minutes Proc. Natl. Acad. Sci. USA 105 476-481

[5] Easley CJ, Karlinsey JM, Bienvenue JM, Legendre LG, Roper MG, Feldman SH, Hughes MA, Hewlett EL, Merkel TJ, Ferrance JP and Landers JP 2006 A fully integrated microfluidic genetic analysis system with sample-in-answerout capability Proc. Natl. Acad. Sci. USA 103 19272-19277

[6] Dongre C, Pollnau M and Hoekstra HJWM 2011 All-numerical noise filtering of fluorescence signals for achieving ultra-low limit of detection in biomedical applications Analyst 136 1248-1251

[7] Martínez Vázquez R, Osellame R, Nolli D, Dongre C, van den Vlekkert HH, Ramponi R, Pollnau M and Cerullo G 2009 Integration of femtosecond laser written optical waveguides in a lab-on-chip $L a b$ Chip 9 91-96

[8] Osellame R, Hoekstra HJWM, Cerullo G and Pollnau M 2011 Femtosecond laser microstructuring: an enabling tool for optofluidic lab-on-chips Laser Photonics Rev. 5 442-463

[9] Crespi A, Gu Y, Ngamson B, Hoekstra HJWM, Dongre C, Pollnau M, Ramponi R, van den Vlekkert HH, Watts P, Cerullo G and Osellame R 2010 Three-dimensional Mach-Zehnder interferometer in a microfluidic chip for spatially-resolved label-free detection Lab Chip 10 1167-1173

[10] Schouten JP, McElgunn CJ, Waaijer R, Zwiinenburg D, Diepvens F and Pals G 2002 Relative quantification of 40 nucleic acid sequences by multiplex ligation-dependent probe amplification Nucleic Acids Res. 30 57-69

[11] Dongre C, van Weerd J, Besselink GAJ, Martínez Vázquez R, Osellame R, Cerullo G, van Weeghel R, van den Vlekkert HH, Hoekstra HJWM and Pollnau M 2011 Modulation-frequency encoded multi-color fluorescent DNA analysis in an optofluidic chip $L a b$ Chip 11 679-683
[12] Alaverdian L, Alaverdian S, Bilenko O, Bogdanov I, Filippova E, Gavrilov D, Gorbovitski B, Gouzman M, Gudkov G, Domratchev S, Kosobokova O, Lifshitz N, Luryi S, Ruskovoloshin V, Stepoukhovitch A, Tcherevishnick M, Tyshko G and Gorfinkel V 2002 A family of novel DNA sequencing instruments based on single-photon detection Electrophoresis 23 2804-2817

[13] Dongre C, van Weerd J, Besselink GAJ, van Weeghel R, Martínez Vázquez R, Osellame R, Cerullo G, Cretich M, Chiari M, Hoekstra HJWM and Pollnau M 2010 High-resolution electrophoretic separation and integrated-waveguide excitation of fluorescent DNA molecules in a lab on a chip Electrophoresis 31 2584-2588

[14] Pollnau M, Hammer M, Dongre C and Hoekstra HJWM 2016 Combined microfluidic-optical DNA analysis with single-base-pair sizing capability Biomed. Opt. Express 7 5201-5207 
16. Nanoscale optofluidics - Sha Xiong and AiQun Liu

Nanyang Technological University

\section{Relevance of the topic}

Over the past decades, optofluidic devices have been extensively exploited for novel functions and promising applications in chemical and biological analysis, imaging and energy by combining the merits of both fluids and optics in the microscale platforms [1, 2]. Recently, researchers have shown great interest in scaling down the channel size and studying both the fundamental and applied science on the nanoscale. When the channel dimension is comparable with the size of molecules, the fluid transport and molecular behaviors are different [3]. Up to now, the most promising applications and phenomena related to nanofluidic devices are based on the electrokinetic effects, such as water desalination, fluidic diodes and energy harvesting. In contrast, the big potential of nanofluidics has not been exploited in the realm of optics. The functions of nanochannels in the optofluidic devices are mainly in two aspects as shown in Fig. 1. First, the nanochannels provide a solution for local tunability over photonic circuits. Their sensitive response to the change of the refractive index can also be used for biosensing. Second, the nanochannels offer effective confinement of biomolecules to facilitate biological analysis, especially for DNA related genomic applications. Despite the great expectation of expanding optofluidics on the nanoscale, it leaves ample room not only for basic discovery but also for technical improvement in current directions.

\section{Current Status and Challenges}

Here, we discuss the state of art and highlight the technical barriers impeding the development of nanoscale optofluidics.

Although enormous progress has been made in the development of nanofabrication over recent years, fabricating well-defined nanochannels with low cost and high throughput is still a challenge. The typical nanofabrication is completed in a well-equipped clean room using a lithography process, such as electronbeam lithography or focused-ion beam lithography. It is usually expensive and laborious with a long processing time. Inspired by the soft lithography with PDMS molding, nano-imprint lithography is employed for large-scale fabrication of nanochannels. Although the replication method improves the throughput, it has limitations in fabricating the nano-molds. Researchers are looking for alternative materials and techniques for fabricating nanodevices, in order to make them commercially viable.

Dynamic reconfiguration of photonic functions is one of the representative topics of optofluidics. It tunes

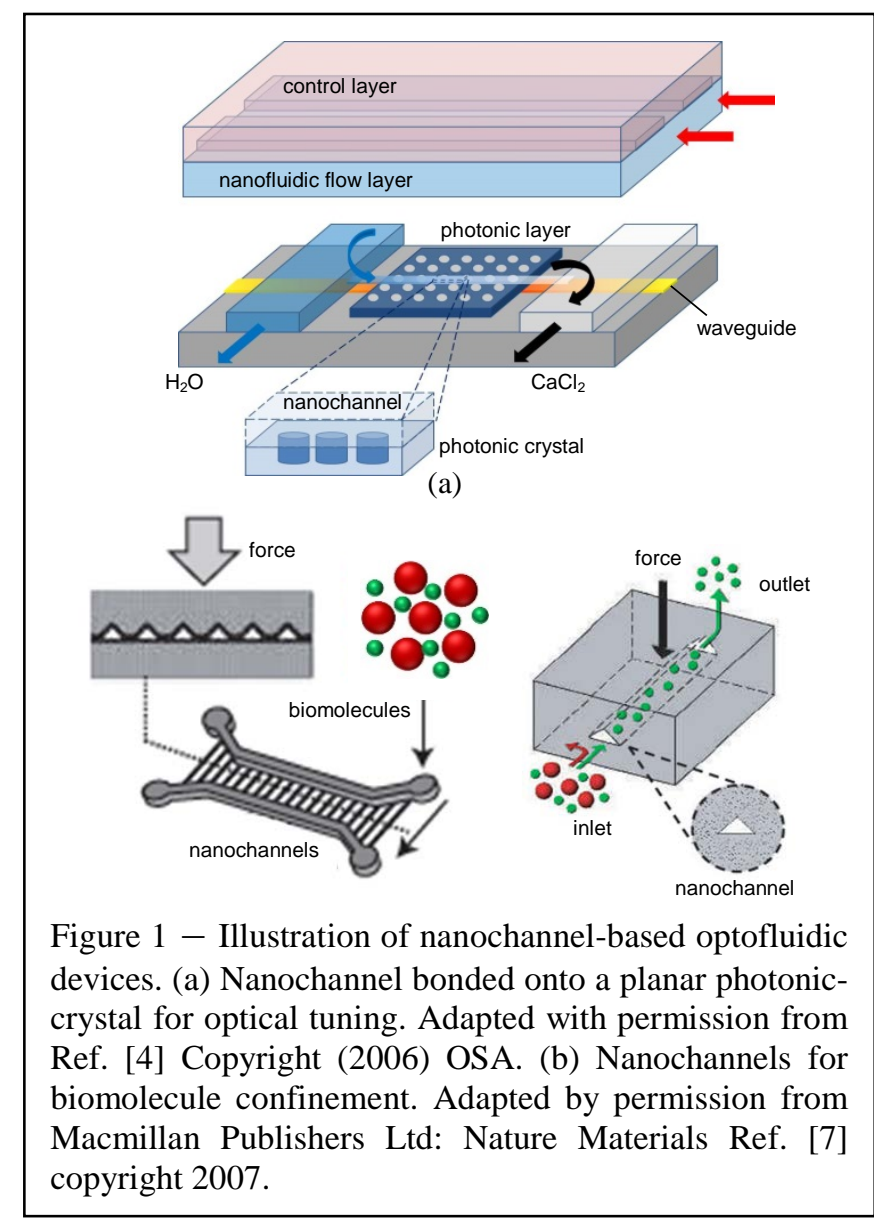

the optical properties of the device by controlling fluid flows with various refractive indices. The nanochannels can infiltrate the liquid into well-defined defects of a photonic crystal [4], or form a tunable grating by filling liquid into the nanochannel array [5]. Introducing the nanofluidic modulation into the photonic circuits provides a powerful approach for realizing compact and reconfigurable optical functionalities with precise localized control. The major challenge for the tuning of photonic functions is the nanofluidic handling. The speed and volumetric flow rate of the liquids transported through the nanochannel is extremely low. Conventional transport methods such as capillary filling, pressure drop or electroosmosis are not effective.

Another hot research topic on nanoscale optofluidics is the analysis and detection of a single molecule. Nanochannels are used to confine the sample volume to match the dimensions of the detection volume, increase its concentration for optical detection, or do precise manipulation of individual molecules, which enhance the sensitivity and resolution of the detection. Researchers have achieved length measurement, sizing and optical mapping of DNA by stretching or separating the molecules in nanochannels [6]. In most cases, the detection depends on the fluorescent labeling, which is costly and timeconsuming. Alternatively, scattering signals can also be used for detecting molecules in the nanochannels, 
which, however, has stringent requirements on the optical detection system. Improving the detection limit remains a bottleneck.

Although new phenomena keep being discovered, it is difficult to precisely probe the transport properties on the nanoscale and get good control over the nanoflow. The existing techniques are mostly based on an electrical measurement. Optical detection may offer alternative strategies, but have to overcome several limitations in nanochannels, such as the influence of Brownian motion and wall effects.

\section{Advances in Science and Technology to Meet Challenges}

A revolution on the nanofabrication is expected, just as the sprout out of microfluidics following the development of PDMS based soft lithography techniques. Recently, mechanical deformation of elastomeric materials (e.g. wrinkling, cracking and microchannel collapsing) was taken advantage of in fabricating nanochannels. These techniques are costeffective and can adjust the channel size by controlling the stress applied on the materials [7]. Meanwhile, wide attention has been drawn towards bottom-up methods, in which the nano-structures are directly assembled by atoms and molecules. Novel materials like carbon nanotubes and graphene have shown their unique properties in nanofluidic transport. One could exploit the new emerging 3D printing technology to fabricate nanochannels in the optofluidic system.

Optical actuation is a burgeoning method for fluid control, which includes optical driven flow through the photo-thermal effect of nanoparticles, direct optical manipulation of particles/structure in the fluid, and laser-induced cavitation, etc. The optical driven nanofluidic transport is insensitive to surface or solution conditions. Even though all the dynamic processes slow down due to the dominating surface forces, it is still possible to generate a high-speed jet by using a pulsed laser to drive flow as shown in Fig. 2 [8].

Besides developing a new optical detection concept and optimizing the optical system, sorting and preconcentration of the targeted samples before detection is a feasible approach to improve the detection limit. Conventional approaches cannot precisely separate the samples. A more robust solution is offered by continuous flow separation of structures such as an entropic trap [9] and nanoscale Deterministic Lateral Displacement (DLD) pillar arrays [10], which successfully demonstrated the sorting of DNA and exosomes. It can enhance the specificity of biomolecules, and therefore increase the sensitivity and specificity of detection. Advanced techniques are expected to increase the throughput of the separation

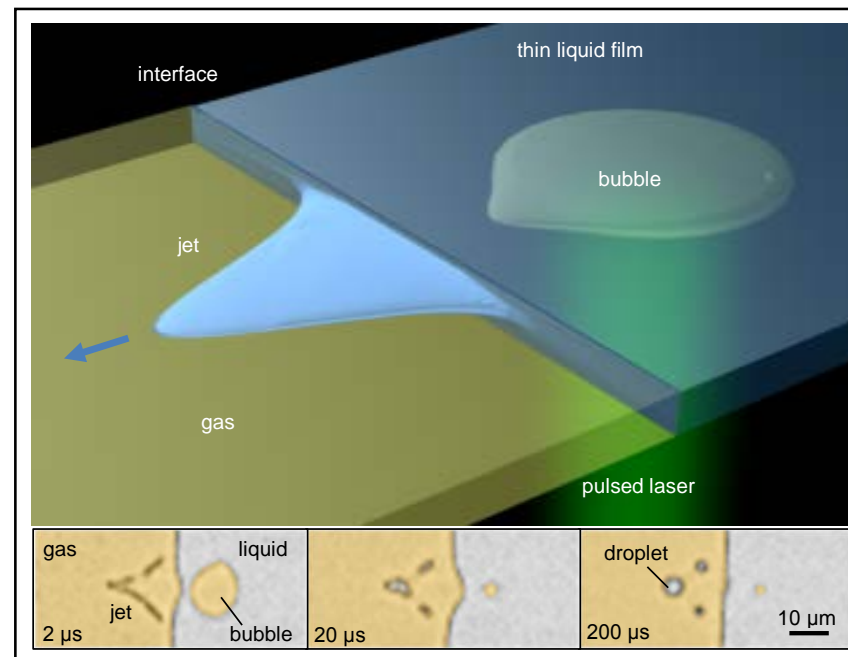

Figure 2 - Jetting caused by the laser at a gas/liquid interface in the nanochannel. Adapted from Ref. [8] with permission from the Royal Society of Chemistry.

and offer sophisticated manipulation on individual molecules.

A better understanding of mass transport at the nanoscale is required to reach the full potential of nanodevices. The next generation of cameras is expected to have higher frame rate and resolution, which is possible to improve the flow characterization in the nanochannels. Combining other imaging techniques such as plasmonics and nanophotonics hold great promise to further improve detection. Moreover, optical induced perturbation on the nanoscale can be an alternative strategy to gain new insight in the flow dynamics.

The integration of nanofluidics and optical components in a fully automated system is a key point for transferring lab-based technologies into the real market. Advanced techniques are expected to integrate the fluidic modulators, light source and optical detection system on the same miniaturized chip, as well as facilitate large-scale production with parallel nanochannels.

\section{Concluding Remarks and Perspectives}

Nanoscale optofluidics is an exciting frontier field, which carries the hope that new opportunities will emerge with the reducing scales. In the point of application, genomic studies are still a hot direction. Its potential in the detection and analysis of exosomes and viruses opens new windows in the diagnostics and environment monitoring. The advanced techniques for optical detection and manipulation should be taken advantage of to go deeper on the fundamental research of nanofluidics such as flow characterization and precise control of flow on the nanoscale. Nanoscale optofluidics has the potential impact to bring breakthrough discoveries, new devices and functions by studying the molecule level interaction. 
Acknowledgments - This work was supported by the Singapore National Research Foundation under its Environmental \& Water Technologies Strategic Research Programme (1102-IRIS-05-01), which is administered by PUB.

\section{References}

[1] Monat C, Domachuk P and Eggleton B J 2007 Integrated Optofluidics: A new river of light Nature Photonics 1 106-114.

[2] Yang Y, Liu A Q, Chin L K, Zhang X M, Tsai D P, Lin C L, Lu C, Wang G P and Zheludev N I 2012 Optofluidic waveguide as a transformation optics device for lightwave bending and manipulation Nature Communications 3651.

[3] Sparreboom W, van den Berg A and Eijkel J C T 2008 Principles and applications of nanofluidic transport Nature Nanotechnology 4 713-720.

[4] Erickson D, Rockwood T, Emery T, Scherer A and Psaltis D 2006 Nanofluidic tuning of photonic crystal circuits Optics Letters 31 59-61.

[5] Yasui T, Ogawa K, Kaji N, Nilsson M, Ajiri T, Tokeshi M, Hriike Y and Baba Y 2016 Label-free detection of real-time DNA amplification using a nanofluidic diffraction grating Scientific Reports 6 31642.

[6] Friedrich S M, Zec H C and Wang T H 2016 Analysis of single nucleic acid molecules in microand nano-fluidics Lab on a Chip 16 790-811.

[7] Huh D, Mills K L, Zhu X, Burns M A, Thouless M D and Takayama S 2007 Tuneable elastomeric nanochannels for nanofluidic manipulation Nature Materials 6 424-428.

[8] Xiong S, Chin L K, Ando K, Tandiono T, Liu A Q and Ohl C D 2015 Droplet generation via a single bubble transformation in a nanofluidic channel $L a b$ on a Chip 15 1451-1457.

[9] Fu J P, Schoch R B, Stevens A L, Tannenbaum S R and Han J Y A 2007 A patterned anisotropic nanofluidic sieving structure for continuous-flow separation of DNA and proteins Nature Nanotechnology 2 121-128.

[10] Wunsch B H, Smith J T, Gifford S M, Wang C, Brink M, Bruce R L, Austin R H, Stolovitzky G and Astier Y 2016 Nanoscale lateral displacement arrays for the speration of exosmes and colloids down to 20 nm Nature Nanotechnology 11 936-940. 
17. Optofluidic immunoassays - Chia-Chann

Shiue and Shih-Kang Fan

National Taiwan University

\section{Relevance of the topic}

Integrating and combining optics and microfluidics, optofluidics [1] has been investigated to achieve precise and adaptive photonic components that generate, shape, route, switch and discriminate light for integrated optical systems. With spatial and temporal control of a fluid on a microscale, the optical properties can be altered and monitored; this capability has been developed further for imaging, light routing, biosensors, energy and other purposes. For applications in biological and chemical analysis, various mechanisms involving photonic detection have been studied in integrated optofluidic systems [2], including metallic nanohole array plasmonics, photonic crystals, photonic crystal fibers, ring resonators, Mach-Zehnder interferometers, and Fabry-Perot cavities to sense the refractive index that are employed as label-free immunosensors. Beyond improving the performance of photonic sensing, the precise and automated manipulations of fluids and suspended particles with microfluidic techniques [3] facilitate assay protocols and enhance biomedical analyses. Among diverse analyses, an optofluidic immunoassay [4] is an essential and common practice in biomedical study and in vitro diagnosis (IVD) to analyze a target analyte, for example proteins and small molecules, by exploiting the sensitivity, specificity and affinity of antibodyantigen interactions.

\section{Current Status and Challenges}

Immunoassays are generally classified as homogeneous or heterogeneous. In homogeneous immunoassays, antigens and antibodies are dispersed and interact in solutions, whereas in heterogeneous immunoassays the target antigens or antibodies suspended in the solution are detected with specific antigens or antibodies immobilized on a solid support surface. Figure 1 shows various heterogeneous immunoassays that occur on a solid surface with appropriately immobilized and labeled antigen or antibody molecules that have been developed. In a competitive configuration, the sensing signal is inversely proportional to the amount of target antibody present in the sample solution. In the non-competitive manners of both sandwich and indirect configurations, a detectable signal from the labeled antibody or secondary antibody is proportional to the amount of target antigen. With optical labels, the signal can be detected with colorimetry, fluorescence, photoluminescence (PL), chemiluminescence (CL) and electrochemiluminescence (ECL) methods simply with a photodiode, photomultiplier tube (PMT), charge-

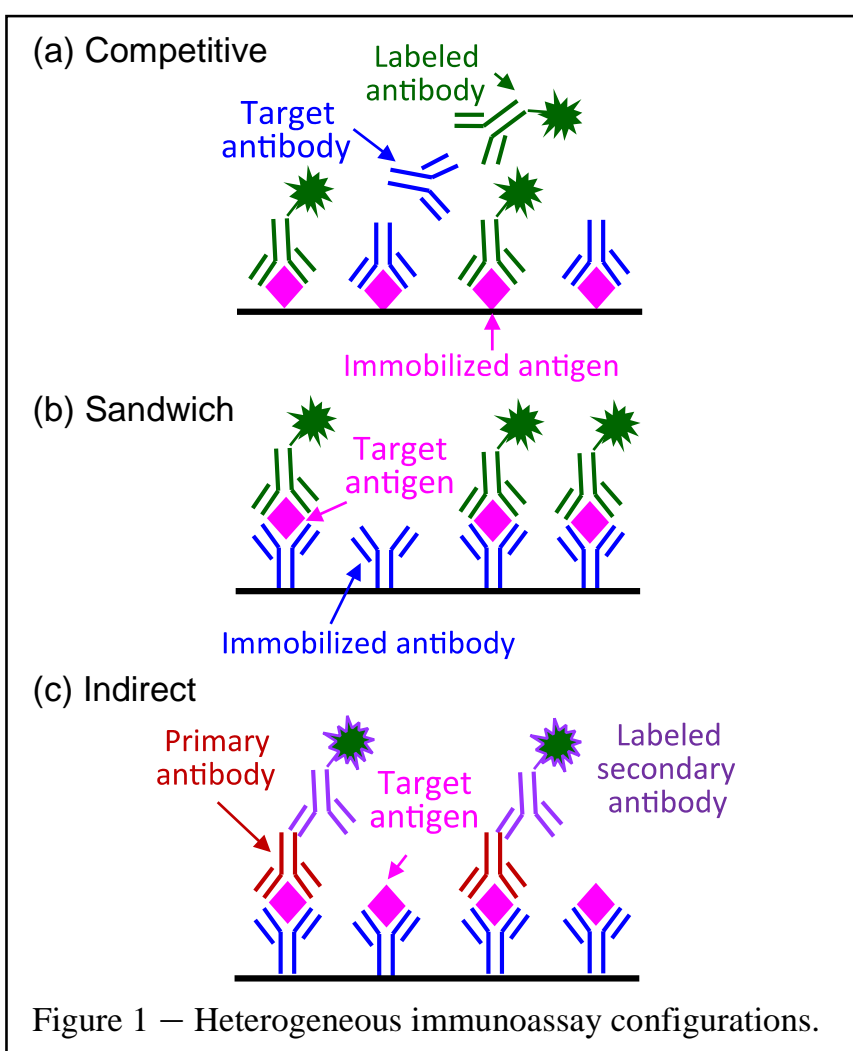

coupled device (CCD) or CMOS image sensor. Without labeled molecules, various optical immunoassays were demonstrated, such as surface plasmon resonance (SPR), interferometers, ring resonators, and photonic crystals [5]. Other non-optical means were achieved with various techniques, for example electrochemical, piezoelectric (quartz-crystal microbalance (QCM) and microcantilever), calorimetric methods and with field-effect transistors (FET).

Concurrently with advances of the antigen/antibody, labeled molecules of immunoassays and optical methods of detection, microfluidic devices drive fluids, liquids or gas, typically along microchannels fabricated on silicon, glass or poly(dimethylsiloxane) (PDMS), to offer a miniaturized, automatic, rapid and cheap analysis with decreased consumption of reagents. Hatch et al. investigated a homogeneous immunoassay conducted along T-shaped microchannels by flowing two fluid streams containing antibody and labeled and target antigen [6]; the diffusion and interaction between the two streams under a laminar flow regime with a small Reynolds number was monitored with a CCD to analyze the concentration of the target antigen. Although the detection was performed within $1 \mathrm{~min}$, the laminar flow maintained by the continuous flow kept consuming the samples and reagents during the measurement. Depending on the tubing between the pump and chip, the dead volume might waste more sample and reagents.

Heterogeneous immunoassays with antigens or antibodies absorbed physically or immobilized 
covalently on a microchannel surface have also been studied. To simplify the fabrication of a microchannel, and modification and immobilization of a surface, conjugated microbeads, especially magnetic particles, have been widely adopted for the development of optofluidic heterogeneous immunoassays that have been applied successfully to clinical samples; to complete the immunoassay, repeated microfluidic procedures, including mixing, incubation, and washing of samples and reagents with precisely metered volumes, are necessary but challenging for microfluidic devices with fixed microchannels that are not reconfigurable during operation.

\section{Advances in Science and Technology to Meet Challenges}

An alternative means to perform optofluidic immunoassay is based on digital microfluidics (DMF) that has demonstrated promising DNA analysis, drug discovery and real-time biomolecular detection with precise, reprogrammable and individual control of multiple droplets. Among techniques of droplet actuation, electrowetting is the most efficient, and has been widely adopted in DMF devices composed of parallel plates free from issues of complicated microchannel fabrication and dead volume. Figure 2 shows how droplets are generated, transported, mixed and split between two plates of glass, silicon or printed circuit board (PCB) on applying electric signals to the electrodes that are covered with a dielectric or hydrophobic layer. For instance, Sista et al. reported a heterogeneous immunoassay to detect insulin and cytokine interleukin-6 (IL-6) on a DMF platform within 7 min [7]; a droplet of sample containing insulin or IL-6 was first mixed with the reagent droplet containing magnetic beads coated with a primary antibody, a secondary antibody labeled with alkaline phosphate (ALP) and blocking proteins. The antibodymagnetic bead-antigen sandwich complex was then collected and fixed on transporting the droplet near a permanent magnet placed either above or beneath the two plates for the following washing steps. The supernatant liquid was removed with droplet splitting; one part of the split droplet containing unbound components was discarded. The magnetic beads in the remaining droplet were resuspended on mixing with an additional fresh washing buffer droplet. The washing was repeated until all unbound components were removed. The CL signal was eventually measured with a PMT on mixing the droplet containing the antibodymagnetic bead-antigen complex with a droplet of CL substrate, Lumigen APS-5. Such a DMF platform capable of automated fluid actuations to conduct multiple assay protocols has been applied to multiplex newborn screening.

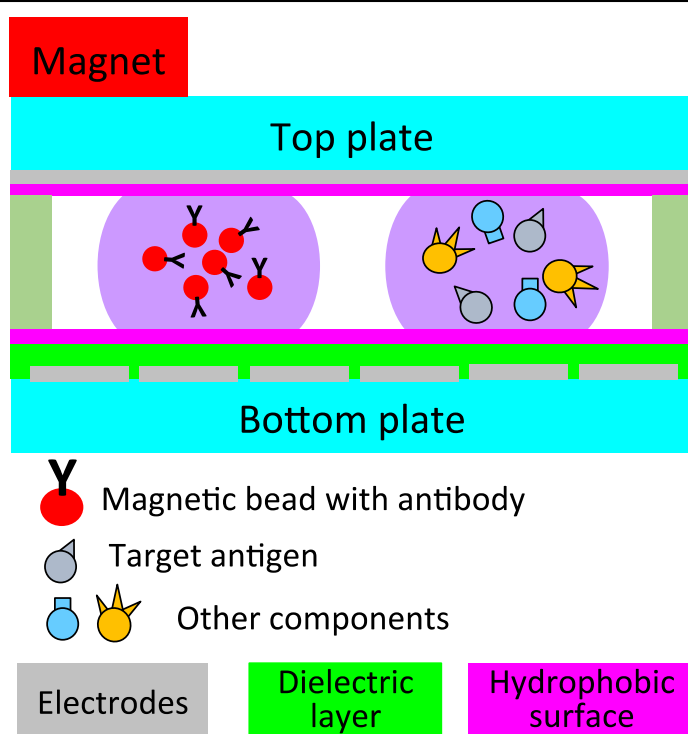

Figure 2 - Parallel plate device configuration of a digital microfluidic (DMF) platform performing magnetic bead-based heterogeneous optofluidic immunoassay with electrowetting driven droplets and precisely metered volumes.

With the sandwich heterogeneous immunoassay configuration using magnetic beads, the fluorescence signal from the fluorophore labeled with secondary antibody was enhanced with appropriately collected and gathered magnetic beads (diameter $6 \mu \mathrm{m}$ ) in a shallow fluidic space (height $10 \mu \mathrm{m}$ ) between plates [8]. The volume of the sample was $2.5 \mathrm{~nL}$; the limit of detection (LOD) was $15 \mathrm{pg} / \mathrm{mL}$ with coefficient of variability (CV) $3 \%$. Moreover, without the top plate a sessile droplet typically performing as a liquid lens was applied to focus the fluorescence on the detector and to enhance the detection sensitivity [9]. The detection was performed in a compact and shielded light-proof box (7 $\times 7 \times 7 \mathrm{~cm}^{3}$ ) with all components, achieving a sensitive, cost-effective and portable optofluidic bioassay system.

\section{Concluding Remarks and Perspectives}

To date, various microchannel-based and droplet-based digital microfluidic techniques have been demonstrated for homogeneous and heterogeneous optofluidic immunoassays. Label-free photonic sensors generally require advanced fabrication techniques and are less suitable for the application of disposable IVD medical devices. DMF with modified magnetic beads to perform bioassays with individually driven droplets simplifies the fabrication and the antigen or antibody immobilization to realize optofluidic immunoassays for clinical diagnostics, enabling the performance of robust fluidic procedures with reliable droplet and magneticbead manipulation without volume variation, bead loss or biofouling. The integration of detectors in the DMF platform [10] to eliminate optical alignment is essential to the development of disposable cartridges for IVD. 


\section{References}

[1] Psaltis D, Quake S R and Yang C 2006 Developing optofluidic technology through the fusion of microfluidics and optics Nature 442 381-386

[2] Fan X and White I M 2011 Optofluidic microsystems for chemical and biological analysis Nat Photonics 5 591-597

[3] Sackmann E K, Fulton A L and Beebe D J 2014 The present and future role of microfluidics in biomedical research Nature 507 181-189

[4] He J-L, Wang D-S and Fan S-K 2016 Optomicrofluidic immunosensors: from colorimetric to plasmonic Micromachines 7 doi: 10.3390/ mi7020029

[5] Fan X, White I M, Shopova S I, Zhu H, Suter J D and Sun Y 2008 Sensitive optical biosensors for unlabeled targets: A review Anal Chim Acta 620 826

[6] Hatch A, Kamholz A E, Hawkins K R, Munson M S, Schilling E A, Weigl B H and Yager P 2001 A rapid diffusion immunoassay in a T-sensor Nat Biotechnol 19 461-465

[7] Sista R S, Eckhardt A E, Srinivasan V, Pollack M G, Palankib S and PamulaV K 2008 Heterogeneous immunoassays using magnetic beads on a digital microfluidic platform Lab Chip 8 2188-2196

[8] Huang C-Y, Shih P-H, Tsai P-Y, Lee I-C, Hsu H-Y, Huang H-Y, Fan S-K and Hsu W2015 AMPFLUID: aggregation magnified post-assay fluorescence for ultrasensitive immunodetection on digital microfluidics Proc IEEE 103 225-235

[9] Zeng X, Zhang K, Pan J, Chen G, Liu A-Q, Fan S-K and Zhou J 2013 Chemiluminescence detector based on a single planar transparent digital microfluidic device Lab Chip 13 2714-2720

[10] Royal M W, Jokerst N M and Fair R B 2013 Droplet-based sensing: Optical microresonator sensors embedded in digital electrowetting microfluidics systems IEEE Sens J 13 4733-4742 
least some form of liquid based sample processing involved. Additionally, optical methods, like florescence, have been used in lab-on-chip devices for many years prior to the advent of the term Optofluidics. As such one could view the goal of the field in this area over the last dozen years as examining ways in which one might be able to: (1) use light and fluidics synergistically or (2) take advantage of advanced photonic/optical methods in ways that lead to better: cost, reliability, deployment, accuracy or outcome advantages than would otherwise be possible.

\section{Current Status and Challenges} provide actionable information to a healthcare provider within a timeframe compatible with the likely patientprovider interaction. A variation on this, which we will focus on here, is point-of-need diagnostics. This differs from point-of-care in an important way, in that the best place for administering the diagnostic may not be where patient care is provided. There are numerous examples of this including: mobile breast cancer screening programs, precision medicine where time tracking of health markers requires both sample collection and analysis to be done in a personalized unit, and infectious diseases monitoring where cases can originate far away from a proper healthcare site and preliminary screening can help ensure referral is done properly. The technologies that enable these diagnostics can exploit chemical, biological or physical measurements of health, be single point measurement or involve monitoring over time, or address acute or chronic health issues.

Optics and Optofluidics for Point-of-Care/Need Diagnostics: Since the early days of what we now call microfluidics, roughly the mid-1990s, point-ofcare/need diagnostics was seen as one of the key applications. The concept was, and is, simple; in the same way that modern integrated electronics enabled mobile, portable computers, microfluidics was going to put the power of a traditional analytical lab into a physician's office through the "Lab-on-a-Chip".

When Optofluidics first emerged as a formal term roughly 10 years later, in the mid-2000s, diagnostics were still viewed as one of the major drivers. Indeed the seminal paper in the field by Psaltis et al. [1] lists an anticipated market demand for the development of "portable devices for environmental monitoring, medical diagnostics and chemical-weapon detection" as one of the key applications Optofluidics is trying to realize.

The need for the "fluidics" part of Optofluidics in diagnostics is fairly obvious in that if one is taking, processing, and analyzing a biological sample (e.g. blood, sweat, saliva, biopsy, etc.) there is likely to be at
There are numerous excellent examples of Optofluidic diagnostic technology including recent work on: diagnostics for concussion recovery [2], rapid single virus detection [3], and minimally invasive malaria diagnostics [4]. Limitations on the length of this article prevent providing a comprehensive review, so rather I will provide two case studies in involving precision medicine and global health. In the final subsection I will point to some needed technological advancements that could have significant impact on the field.

\section{Case Study I - Optofluidics for Precision Medicine}

The US National Institutes of Health defines Precision Medicine as "an emerging approach for disease

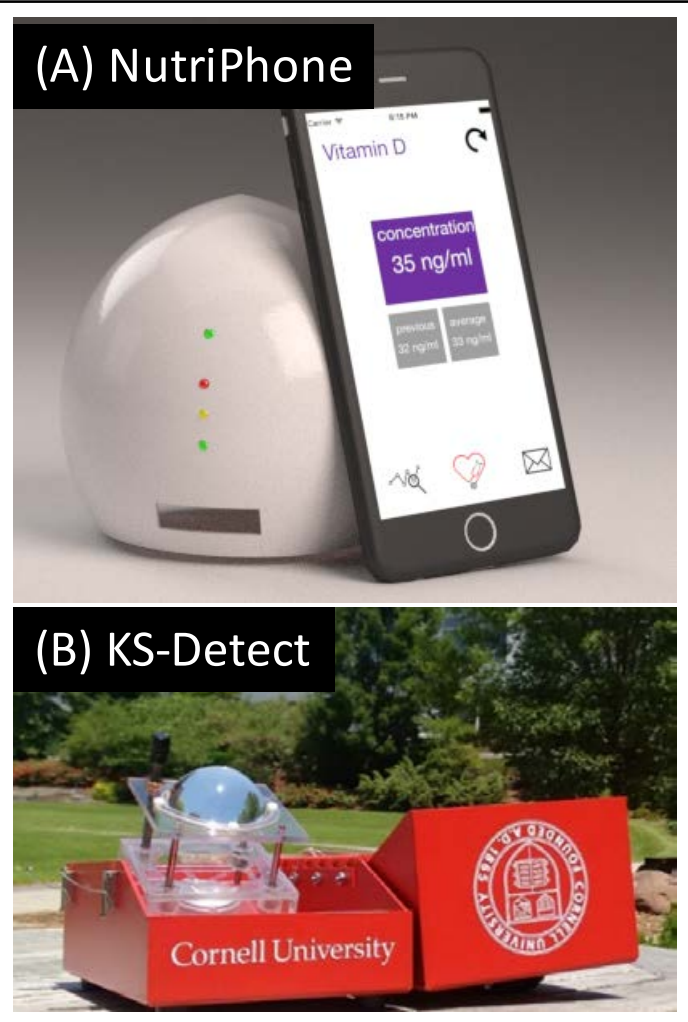

Figure 1 (A) NutriPhone - Personalized Nutrition Tracking and (b) KS-Detect - SolarThermal PCR. 
treatment and prevention that takes into account individual variability in environment, lifestyle and genes for each person.” There are a number of ways in which Optofluidics can play a number of roles in precision medicine with the most relevant to this article being to enable the point-of-need diagnostics that can enable the better, easier, and more accurate tracking of biomarkers at the individual level. Being able to fuse biomarker data with changes in behavior or environment is key to understanding how individuals react.

Given the current worldwide near-ubiquity of mobile technology, it is reasonable to pair these types of diagnostics with mobile technologies, such as mobile phones and tablets [5]. As one example, our group and the Mehta group in Nutritional Sciences at Cornell have been working on coupling Optofluidics based micronutrient level tracking diagnostics with mobile phones. Our "NutriPhone", see Figure 1(A), uses a specially developed paper microfluidics card coupled with an Optofluidic reader system to enable individuals to easily track how changes in diet and lifestyle can affect circulating levels of different micronutrients. Monitoring nutritional outcomes is a particularly good candidate for personalized medicine because responses to changes in diet and lifestyle can be very individualized and often difficult to quantify the magnitude of changes a priori. To date we have developed tests for vitamin $\mathrm{D}\left(25-\mathrm{OH}-\mathrm{D}_{3}\right)$ [6], iron (ferritin), vitamin $\mathrm{B}_{12}$ [7], and others.

\section{Case Study II - Optofluidics for Global Health}

Formally "Global Health" refers to the health of everyone on the planet, independent of country of origin. When used in the context of technology development, however, it often refers to systems for use in so-called "limited resource settings" or places where access to reliable infrastructure ideally required to operate the technology may not be available. In 2012 our group published a paper broadly describing the Optofluidic opportunities within Global Health [8], including point-of-care diagnostics.

Numerous agencies and foundations have published criteria for what is required for a successful point-ofneed diagnostic in limited resource settings. These requirements vary slightly depending on the source but most include requirements for devices to be: operable in locations with limited or no medical infrastructure, simple to operate and interpret, and able to provide actionable information with fidelity similar to what one could expect with the state-of-the-art test. Coupled with these technical requirements, there is an equally important need that the technology be coupled with a sustainable business/deployment model. This means that the system and any required consumables need to be able to be manufactured, serviced, distributed, and operated in a way that is compatible with the existing healthcare system. Since these requirements (including but not limited to price-point) vary from location to location, it is key to involve local expertise as early on in the process as possible.

An example of how we have used Optofluidics to address some of these concerns is our KS-Detect system [9, 10] (see Figure 1B). The technology focuses solar or other light through a shadow mask onto an optically absorbing microfluidic chip. The system is designed such that the projection of this light onto the chip creates a radial thermal pattern in steps whose temperatures match those required to conduct a PCR reaction. The channels on the microfluidic chip are designed so as to move the sample through the static temperature zones with the proper residence time. This technique, coupled with the use of a mobile phone to read and interpret the results, allows the device to be operated under conditions where power may not be available or reliable. This enables one to have more flexibility in performing the diagnosis at the true point-of-need rather than just the point-of-care. As can be seen in the above mentioned papers, we have demonstrated the technology for the diagnosis of Kaposi’s Sarcoma.

\section{Advances in Science and Technology to Meet Challenges}

There are numerous opportunities for Optofluidic technology in point-of-need diagnostics. The key technological and scientific advancements that will likely be required include: further and more seamless integration of Optofluidic systems with mobile technology, reduction or elimination of the need for peripheral equipment needed to operate technology (e.g. tunable lasers), reduced material and fabrication costs for consumables to hit consumer-level or internationally acceptable price points, and an increased emphasis on the skill set of the end user resulting in lower complexity easier to use devices and systems.

\section{Concluding Remarks and Perspectives}

A transformative change would be the ability to use Optofluidic or optical/spectroscopic methods to reduce the invasiveness of diagnostics. This has proven difficult in the past due to challenges with accuracy and high per-unit cost of the resulting instrumentation and thus technologies that can address these two challenges would be highly desirable.

Acknowledgments - DE acknowledges support for his efforts in the areas discussed above from the US National Institutes of Health through grants R01-EB021331 (FeverPhone) and UH2-CA-202723 (KS- 
Detect) and the US National Science Foundation through grants CBET-1343058 (Mobile Health) and IIP-1430092 (NutriPhone).

The author declares the following competing financial interests(s): DE has equity interest in VitaMe Technologies Inc. which is commercializing micronutrient diagnostic technology similar to that described herein.

\section{References}

1. $\quad$ Psaltis, D., S.R. Quake, and C.H. Yang, Developing optofluidic technology through the fusion of microfluidics and optics. Nature, 2006. 442(7101): p. 381-386.

2. Ko, J., et al., Smartphone-enabled optofluidic exosome diagnostic for concussion recovery. Scientific Reports, 2016. 6.

3. Ozcelik, D., et al., Optofluidic wavelength division multiplexing for single-virus detection. Proceedings of the National Academy of Sciences of the United States of America, 2015. 112(42): p. 12933-12937.

4. Lukianova-Hleb, E.Y., et al., Hemozoin-generated vapor nanobubbles for transdermal reagent- and needle-free detection of malaria. Proceedings of the National Academy of Sciences, 2014. 111(3): p. 900-905.

5. Erickson, D., et al., Smartphone technology can be transformative to the deployment of lab-on-chip diagnostics. Lab on a Chip, 2014. 14(17): p. 31593164.

6. Lee, S., et al., A smartphone platform for the quantification of vitamin $D$ levels. Lab on a Chip, 2014.

7. Lee, S., et al., NutriPhone: a mobile platform for low-cost point-of-care quantification of vitamin B12 concentrations. Under review, 2016.

8. Chen, Y.-F., et al., Optofluidic opportunities in global health, food, water and energy. Nanoscale, 2012. 4(16): p. 4839-4857.

9. $\quad$ Snodgrass, R., et al., KS-Detect - Validation of Solar Thermal PCR for the Diagnosis of Kaposi's Sarcoma Using Pseudo-Biopsy Samples. Plos One, 2016. 11(1): p. e0147636.

10. Jiang, L., et al., Solar thermal polymerase chain reaction for smartphone-assisted molecular diagnostics. Sci. Rep., 2014. 4. 


\section{Optofluidics in energy - David Sinton University of Toronto}

\section{Relevance of the topic}

With broad economic and environmental impacts, the energy sector is at the forefront in the minds of policy makers, technology developers and the general public. The dominant demand for global energy usage is in fluids - oil and gas - and the ultimate source of global energy is light - solar radiation. The tight integration of light and fluids via optofluidics thus presents various opportunities for current and future energy technologies. The smallscale of traditional optofluidics technologies, however, is in stark contrast with the scale required for impact in the energy sector. Thus, as with microfluidics for energy applications, the challenge here is to develop optofluidic technologies that can leverage the integration of light and fluids fundamental to the area, while enabling scaling of the technology. The two established routes to scale are: leveraging already-scaled materials such as fiber optics (energy or energy conversion is the product); and employing optofluidics to inform processes at larger scales (information is the product).

An early vision for the field of optofluidics for energy was mapped in our perspective paper five years ago [1]. My focus here is the recent developments in these areas, newly emerging energy applications and the roadmap ahead to broader application of optofluidics in energy. Specifically, I outline first recent progress and a roadmap for optofluidics in photosynthetic and photocatalytic energy conversion - examples of tight integration of fluids and light - followed by emerging and hybrid approaches that leverage elements of the optofluidics toolbox for application in energy. In this short overview, I unfortunately cannot do justice to other exciting developments in this arena such as liquid lensing for solar energy collection, photothermochemical conversion, and solar steam generation, to name a few.

\section{Current Status and Challenges}

Photosynthesis is nature's method of converting light energy into chemical energy, and is well-suited to optofluidics [1-2]. Most of the challenges in this approach stem from the fundamental low efficiency of the photosynthetic process - effectively the overhead associated with biological machinery in series with losses of light energy at both the reactor scale and the organism scale. Although these challenges can be overcome to varying degrees with engineering, implementation increases cost in an already-strained sector [3]. Fuel production represents the largest volume potential market for photobioreactor products, but it is also provides the lowest margin. The low price of conventional hydrocarbons made biofuels a challenging market, and the economics became bleaker with the advent of unconventional oil and gas made possible by the

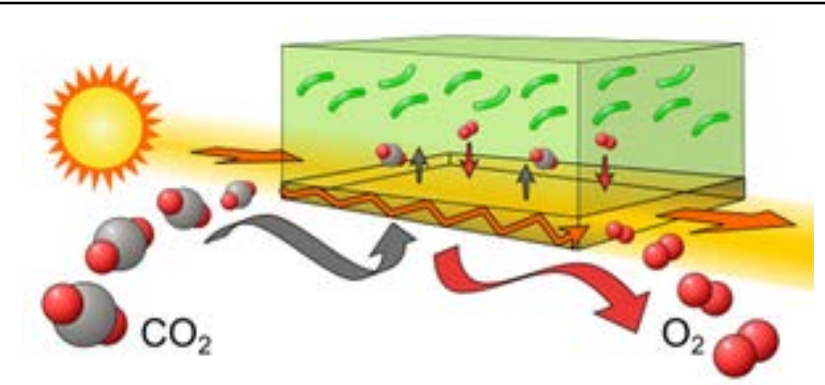

Figure 1 - Breathable waveguides for combined light and fluid delivery to photosynthetic microorganisms. Reproduced from Pierobon et al. [10].

combination of horizontal drilling and hydraulic fracturing in shale and tight oil reservoirs. As a result, biofuel producers and technology developers are focusing on niche high-value chemical outputs, such as neutraceuticals, in which optofluidics photobioreactors can better compete. Optofluidics has also enabled very controlled multiplexed experiments to determine optimal conditions for photosynthetic energy conversion in operations of all scales [4], [5].

Converting light energy into chemical energy using photocatalysts is an alternative to the biological approach. Integrating photocatalysts and fluid in optofluidic systems presents several attractive features, most notably enhanced transport of reactants to photocatalysts and products away, as well as leveraging the excellent control associated with optofluidics systems to ensure conditions throughout [6]. Analogous to the photosynthetic energy conversion (above), however, there are challenges of both cost and efficiency for optofluidics photocatalysis. Specifically, there is a mismatch between the goals of light collection (favoring large areas) and conversion (favoring dense reactors). This mismatch can be technologically addressed by separately collecting and concentrating solar energy and feeding light to, and distributing within, a dense reactor system using waveguides. There are associated challenges of minimizing losses and costs in this waveguiding approach, but there are exciting developments in this area.

Separating light collection and reactor functions by using conventional solar photovoltaics to collect and convert solar energy into electrical energy is another approach [7]. The result is not the tight integration of light and fluids germane to optofluidics, but the advantages are worth considering. Most notably, this approach leverages the massive scaling and cost reductions already achieved in solar photovoltaics, as well as the ease with which electrical output can be controlled and distributed. The fluids-half of the challenge is then in designing effective electrochemical reactors for which there is a long history from fuel cells and electrolyzers. Electrocatalytic conversion of $\mathrm{CO}_{2}$ into (or back into) useful chemical feedstocks using solar-generated renewable electrons is an attractive approach that is gaining traction - 
particularly with advancements in both nanostructure electrocatalysts [8] and gas diffusion electrode cell designs [9].

\section{Advances in Science and Technology to Meet Challenges}

In the area of optofluidics photobioreactors, a promising approach is unifying light and fluid delivery within single materials, such as light-guiding gas-permeable membranes illustrated in Figure 1 [10]. This approach removes the necessity for separate light/fluid infrastructure and associated costs. The next logical step in this progression of simplification might be to integrate immobilized photosynthetic organisms within such materials. Challenges of this approach are many, particularly as the one material must serve many separate functions, but there is an opportunity for cost reductions through the integration. A less exotic and more accessible approach would be to improve the optical function of existing medium- or large-scale facilities, by for instance, providing excitation light that is offabsorption-peak. Providing light that matches the absorption peak of the photosynthetic organism is a common practice, which is grounded in the idea of feeding the organism the light it is tuned to absorb best. The result, however, is rapid absorption of light by the culture and very poor light distribution resulting in poor light use efficiencies overall. The penetration of various wavelengths into identical cultures is shown in Figure 2. Illuminating cultures with wavelengths that are off-peakabsorbance, can allow much deeper penetration of the exciting light while maintaining photosynthetic productivity and boosting overall performance [11].

Photocatalytic reactors have made impressive advances over the last few years - both in the context of wastewater treatment and light-to-chemical energy conversion. The photocatalysis community has the challenge of engineering catalysts that efficiently manage both light utilization and chemical conversion while minimizing electron-hole recombination, bandgap, activity loss, toxicity and material cost. Despite these fundamental challenges there is much progress and reason for optimism in this area. Analogous to photosynthesis, there are also roles for optofluidics systems in informing both the synthesis of photocatalysts and how they might be used in larger systems, such as reactors with disperse photocatalytic nanoparticles [12].

Hybrid methods that separate solar-PV and electrocatalytic conversion have challenges to address particularly in the electrochemical reactions and reactors. Electrocatalytically upgrading $\mathrm{CO}_{2}$ is energy intensive and advances are needed in catalysts and cells to increase Faradaic efficiency for intended products, increase current densities, and reduce the cost of catalysts. A challenge shared with photocatalysis is the production of long-chain hydrocarbons from $\mathrm{CO}_{2}$ (something

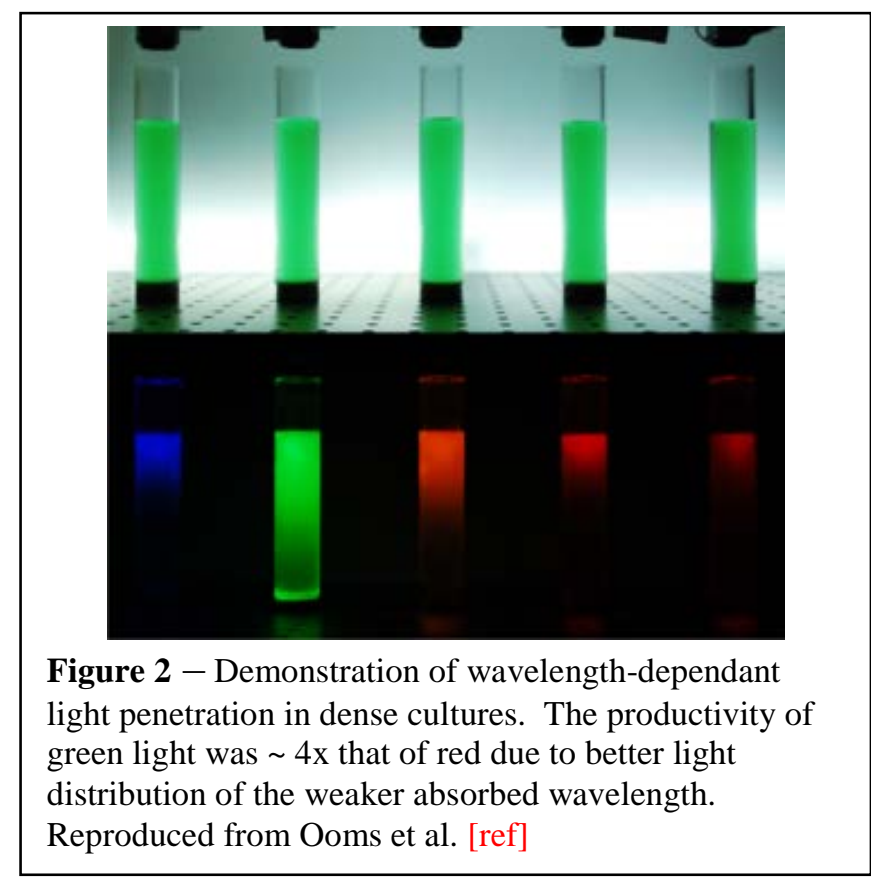

photosynthesis accomplishes). Although these long-chain liquid hydrocarbons are the preferred energy currency worldwide, producing them from $\mathrm{CO}_{2}$ is a veritable, multistep photo/electrochemical challenge. Particularly with the increase in activity in this area of late, there is great potential for rapid advances in here short order.

The role of optofluidic systems to study and inform on photosynthetic and photocatatlyic processes is noted above. We see additional, broader opportunities for this approach here. Specifically, these same methods are very well suited to quantify the environmental impacts of anthropogenic $\mathrm{CO}_{2}$ emissions on organisms and microcosms, in combination with light variables and/or a variety of local stressors. One can imagine independently controlled optofluidic reactors as multiplexed microgreenhouses, or micro-ecosystems, that can provide the massive amount of data necessitated by fundamentally complex, multivariable coupled interactions of the natural environment.

\section{Concluding Remarks and Perspectives}

A major barrier to wider application of renewable energy is the lack of storage. The optofluidic approaches outlined here aim to overcome this barrier by converting - directly or in hybrid - our most plentiful energy source, solar energy, into our most demanded energy form, liquid hydrocarbons. Converting these concepts and demonstrations into useful, scalable approaches will take a sustained effort from the science and engineering communities, as well as funding from policy makers and venture capital. This short overview covered some recent developments and opportunities in photosynthetic, photocatalytic and hybrid solar-PV-electrocatalysis approaches. All three of these approaches have shared challenges of cost and present opportunities for both fundamental science and engineering advancement, the 
most promising of which will keep cost minimization as 2107-2115, 2015. the guiding and motivating principle.

Acknowledgments - We gratefully acknowledge support from the NSERC E.W.R. Steacie Memorial Fellowship, Discovery program, MEET CREATE program, and infrastructure funding from CFI.

\section{References (separate from the two page limit)}

[1] D. Erickson, D. Sinton, and D. Psaltis, "Optofluidics for energy applications," Nat. Photonics, vol. 5, no. 10, pp. 583-590, Sep. 2011.

[2] S. S. Ahsan, A. Gumus, A. Jain, L. T. Angenent, and D. Erickson, "Integrated hollow fiber membranes for gas delivery into optical waveguide based photobioreactors," Bioresour. Technol., vol. 192, pp. 845-849, 2015.

[3] M. D. Ooms, C. T. Dinh, E. H. Sargent, and D. Sinton, "Photon management for augmented photosynthesis," Nat. Commun., vol. 7, p. 12699, Sep. 2016.

[4] P. J. Graham, J. Riordon, and D. Sinton, "Microalgae on display: A microfluidic pixel-based irradiance assay for photosynthetic growth," Lab Chip, vol. 15, no. 15, pp. 3116-3124, 2015.

[5] B. Nguyen, P. J. Graham, and D. Sinton, "Dual gradients of light intensity and nutrient concentration for full-factorial mapping of photosynthetic productivity," Lab Chip, vol. 16, pp. 2785-2790, 2016.

[6] S. S. Ahsan, A. Gumus, and D. Erickson, "Redox mediated photocatalytic water-splitting in optofluidic microreactors,” Lab Chip, vol. 13, no. 3, pp. 409-414, 2013.

[7] C. A. Rodriguez, M. A. Modestino, D. Psaltis, and C. Moser, "Design and cost considerations for practical solar- hydrogen generators," Energy Environ. Sci., vol. 7, pp. 3828-3835, 2014.

[8] M. Liu, Y. Pang, B. Zhang, P. De Luna, O. Voznyy, J. Xu, X. Zheng, C. T. Dinh, F. Fan, C. Cao, F. P. G. De Arquer, T. S. Safaei, A. Mepham, A. Klinkova, E. Kumacheva, T. Filleter, D. Sinton, S. O. Kelley, and E. H. Sargent, "Enhanced electrocatalytic CO2 reduction via field-induced reagent concentration," Nature, vol. 537, no. 7620, pp. 382-386, 2016.

[9] H. M. Jhong, S. Ma, and P. J. Kenis, "Electrochemical conversion of $\mathrm{CO} 2$ to useful chemicals: current status, remaining challenges, and future opportunities," Curr. Opin. Chem. Eng., vol. 2, no. 2, pp. 191-199, May 2013.

[10] S. C. Pierobon, J. Riordon, B. Nguyen, and D. Sinton, "Breathable waveguides for combined light and CO2 delivery to microalgae," Bioresour. Technol., vol. 209, pp. 391-396, Jun. 2016.

[11] M. D. Ooms, P. J. Graham, B. Nguyen, E. H. Sargent, and D. Sinton, "Light dilution via wavelength management for efficient high-density photobioreactors," Biotechnol. Bioeng., vol. xx, p. (under review), 2017.

[12] T. Burdyny, J. Riordon, C.-T. Dinh, E. H. Sargent, and D. Sinton, "Self-assembled nanoparticlestabilized photocatalytic reactors.," Nanoscale, pp. 